\title{
ResearchOnline@JCU
}

This is the author-created version of the following work:

Langley, Michelle C., Clarkson, Chris, and Ulm, Sean (2019) Symbolic expression in Pleistocene Sahul, Sunda, and Wallacea. Quaternary Science Reviews, 221 pp. 1-22.

Access to this file is available from:

https://researchonline.jcu.edu.au/59887/

Please refer to the original source for the final version of this work: 


\section{Symbolic Expression in Pleistocene Sahul, Sunda, and Wallacea}

Michelle C. Langley ${ }^{\mathrm{a}^{*}}$, Chris Clarkson ${ }^{\mathrm{b}, \mathrm{c}}$ and Sean Ulm ${ }^{\mathrm{d}}$

a Australian Research Centre for Human Evolution, Environmental Futures Research Institute, Griffith University, Nathan, Queensland, 4111, Australia m.langley@griffith.edu.au

b School of Social Science, The University of Queensland, Brisbane, Queensland, 4072, Australia c.clarkson@uq.edu.au

c ARC Centre of Excellence for Australian Biodiversity and Heritage, University of Wollongong, New South Wales, 2522, Australia

d ARC Centre of Excellence for Australian Biodiversity and Heritage, College of Arts, Society and Education, James Cook University, PO Box 6811, Cairns, Queensland 4870, Australia sean.ulm@jcu.edu.au

* Corresponding author 


\begin{abstract}
The pace of research undertaken in Sunda (Southeast Asia) through to Sahul (Greater Australia) has increased exponentially over the last three decades, resulting in spectacular discoveries ranging from new hominin species, significant extension to the age for first human occupation in the region, as well as the identification of what is currently the oldest known rock art in the world. These breakthroughs cast the archaeological record of complexity in Sunda, Sahul, and Wallacea in an entirely different light to that of several decades ago, placing it on an equal footing to that of Africa, Asia, and Europe. The archaeological record of these regions now points to rich and diverse early modern human (Homo sapien) societies engaged in complex symbolic and technological behaviours demonstrating capacities for innovation and selfexpression found in all modern human groups now around the globe. Here we provide a comprehensive review of all Pleistocene symbolic evidence reported for Sahul, Sunda, and Wallacea to date. We explore how recent findings have changed our perceptions of the first modern human colonists and our understanding of the origins and development of the rich and diverse cultures that arose in each region through time.
\end{abstract}

\title{
Keywords
}

cognition; ornamentation; art; information exchange; colourants; modern human; modern human behaviour

\section{Highlights}

- We review all evidence for Pleistocene symbolic behaviour in Sahul, Sunda, Wallacea.

- We explore how recent findings have changed our perceptions of these regions.

- We overturn long-held notions that Sahul, Sunda, and Wallacea were static and lacked cultural dynamism. 


\section{Introduction}

Of all our capabilities, it is our modern capacity for 'symbolic' behaviour that is most celebrated. Indeed, our "ability to represent objects, people, and abstract concepts with arbitrary symbols, vocal or visual, and to reify such symbols in cultural practice" (McBrearty and Brooks, 2000:492) has emerged as one of the few universally accepted avenues for investigating cognition in the deep past (e.g., Chase and Dibble, 1987; McBrearty and Brooks, 2000; Wadley, 2001; Klein and Edgar, 2002; d'Errico, 2003; d'Errico et al., 2003; Henshilwood and Marean, 2003; Hovers et al., 2003; Conard, 2008). The continued importance of symbolism in constructing understandings of human cognitive development demands that researchers stay up-to-date on what is known for regions across the Old World. Over the past decade, increasing discoveries unveiled in Island Southeast Asia (ISEA) and northern Australia (i.e., Aubert et al., 2014; Langley et al., 2016a, 2016b; Brumm et al., 2017; Clarkson et al., 2017; Aubert et al., 2018) have resulted in Sahul (the combined landmass of Australia, New Guinea and Tasmania at times of lower sea level), Sunda (Southeast Asia), and Wallacea (the islands in between) becoming increasingly central to academic discussions revolving around modern human cultural development.

Previous reviews of symbolic elements (artefacts and features) dating to the earliest periods of Homo sapien colonisation of Sahul and its closest neighbours, along with their implications for our understanding of these regions, are now outdated. Here we provide: (1) a review of all Pleistocene symbolic evidence reported to date for Sahul, Sunda, and Wallacea, (2) describe how recent findings have changed our perceptions of the first cultures of these regions, and (3) discuss their implications for current understandings of the development of the diverse and rich cultures observed amongst ancient communities in these regions.

\section{Previous Understandings: Symbols, Sahul, Sunda, and Wallacea}

Symbolically-mediated behaviour is its ability to store and display of information external to the human brain (Donald, 1991; Wadley, 2001; Henshilwood and Marean, 2003; Hovers et al., 2003). This ability enables individuals and groups to communicate and record enormous amounts of information, underwriting the development of the rich and diverse cultures now found around the globe. 'Symbolic artefacts' are the material representation of this behaviour, and it has been argued that such items were effectively the 'glue' that held together larger and more complex, fully 'modern' social groups.

The most effective signs and symbols are those that are easily interpretable and have meaning for both the receiver and the emitter; for example, clothing is one of the more discernible symbols that people look to for displaying identity in intergroup interactions (Kuper, 1973; Wobst, 1977; Cordwell and Schwarz, 1979), as is ornamentation (Wiessner, 1983, 1984; O'Hanlon, 1989; White, 1992; Sciama and Eicher, 1998; Kuhn and Stiner, 2007a, 2007b), and the use of colour (d'Errico, 2003; Taçon, 2008). Other means of social selfexpression include gestures, dance, music, and body posture (Barth, 1969; Stevenson, 1989). Material things including portable art, distinctive weaponry, or other items which may display 'style' through its overall morphology or markings (made with colourants, engravings, etc.), and which are likely to be seen by both ingroup and outgroup members are also demonstrated to be highly effective symbols (Conkey, 1980; Wiessner, 1983, 1984; Lemonnier, 1992; Carr, 1995; Taçon, 2008; see Tostevin, 2007 for discussion).

In many ethnographic hunter-gatherer societies, these items reflect the status and affiliation of an individual, and "their use requires an intimate and often tacit knowledge of the cultural and social system"' (d'Errico 
1998:28; see Strathern and Strathern, 1971; Faris, 1972; Kuper, 1973; Wobst, 1977; Brain, 1979; Hodder, 1979; Cordwell and Schwarz, 1979; Dubin, 1987; Hodder, 1991; Sciama and Eicher, 1998; Kuhn and Stiner, 2007a, 2007b). As Wiessner (1984) has argued, it is a fundamental human condition to build personal and social identity through comparison and, consequently, style is not just a means of transmitting information about identity, but is an active tool used in building social strategies. Furthermore, these artefacts have also been shown to play a role in boundary maintenance which, in itself, implies a connection of a specific landscape with its people, its resources, and its stories - access to which needs to be mediated with those who claim a bond to that place (Wobst, 1977; Wiessner, 1983, 1984).

The identification and consideration not only of the 'symbolic' artefacts themselves, but also their distribution in time and space, offers avenues for investigating and understanding past social organisation and cultural customs. Symbolic artefacts and features in the archaeological record reflect not only the ability of a population to use abstract concepts, but also the deployment of these symbolic concepts to link individuals and populations to their technologies and environments. This effective linking of people with technology, and people with people, is argued to ultimately have allowed modern humans to out-perform archaic hominin populations across the Old World (Conard, 2008). Similarly, the symbolically-driven relationship between people and landscapes is suggested to be a qualitative difference between modern humans and near contemporary Neanderthals (Langley, 2013). Currently, Homo sapiens are known to be anatomically modern by 315,000 years ago (Hublin et al., 2017), with the first signs of symbolic behaviour emerging around 164,000 years ago (Marean et al., 2007, Watts, 2010), close to the time modern humans first left Africa (Hershkovitz et al. 2018; Harvati et al. 2019).

Focused examination of Pleistocene symbolic behaviour in Sahul only commenced when researchers began to explore the origins and development of 'behavioural modernity' in Homo. Up until this point, the 1970s reporting of the Lake Mungo burials (Bowler et al., 1970, 1972) was the sole Australian evidence commonly cited in global discussions of early symbolic human behaviour. These spectacular burials remain important in the Sahul story, with the LM1 individual remaining the oldest human cremation in the world. It was only in the mid-to-late 2000s that researchers began to articulate evidence from other regions with the global debate. Indeed, many Australian authors attempted to move the focus on early modern human symbolism away from Europe to Australia (e.g., Brumm and Moore, 2005; Hiscock and O'Connor, 2006; Balme et al., 2009; Langley et al., 2011; Dennell and Porr, 2014 and chapters therein). Davidson and Noble (1992:135) led the way by outlining the reasons for why the initial colonisation of Australia could be considered "the oldest evidence for the expression of behaviour that is distinctively human" or "the earliest documentation of the evolutionary emergence of language" (see Bird et al., 2018, 2019). Following this contribution, the majority of studies mentioning 'symbolism' in connection to Sahul involved a focused theoretical exploration of a single artefact category or behaviour commonly used to identify 'behavioural modernity' in Eurasian and/or African contexts. For example, Davidson (2003), and later Hiscock and O'Connor (2005, 2006), focused on the temporal and regional distribution of blades in Australia in order to demonstrate that such technology lacked theoretical grounds for inclusion on behavioural modernity trait lists. Similarly, Cosgrove and PikeTay (2004) demonstrated that using particular subsistence strategies may also be problematic for identifying behavioural modernity in the past They illustrated this by exploring how the subsistence practices of Pleistocene Tasmanians more closely resembled the European 'archaic' Middle Palaeolithic record than the 'modern' Upper Palaeolithic. Also offered in this comparative context were discussions on evidence for starch processing found on grindstones (Fullagar, 2006), marine shell beads (Balme and Morse, 2006), and clothing (Gilligan, 2007a, 2007b, 2007c). Each study either emphasised strong similarities or differences with the intensively studied Eurasian and African archaeological records. 
Several studies went one step further in pursuing overarching discussions of the variety of evidence for behavioural modernity found in Sahul's Pleistocene archaeological record (Davidson and Noble, 1992; Mellars, 2006; Davidson, 2007; O'Connell and Allen, 2007; Smith et al., 2007; Balme et al., 2009). These studies drew together a wider range of evidence, but provided limited substantive quantitative data to support their hypotheses and conclusions. Short tables listing examples of archaeological evidence were common and used to demonstrate the synchronic presence of a particular artefact category or behaviour within the Pleistocene archaeological record. Specific mentions of the oldest examples of specific artefact classes (e.g., ground-edge axes, pigment, rock art, beads) characterise these papers. Similarly, what little evidence for early symbolic material culture was known at that time from Sunda, was glossed over in deference to the 'better' evidence for behavioural modernity — the colonisation of Sahul (e.g., Mellars, 2006; Balme et al., 2009). The only real exception to this trend, was in discussions of the evidence provided by the Niah Caves, Sarawak, Borneo, which garnered more focused attention (e.g., Hunt and Barker, 2014), while others made generalised statements about the general lack of symbolic evidence in the region. For example, Simanjuntak et al. (2015:165) stated that "symbolic behaviours such as the use of personal ornaments and pigment, rock painting, and ceremonial burial practices ... seldom appear in the Pleistocene archaeological record of Indonesia".

Up to 2011, only three studies offered any quantitative analysis of the evidence for behavioural modernity in Sahul. Brumm and Moore (2005) tested the implication that the earliest colonisers of Australia participated in the 'symbolic revolution' - that is, were 'behaviourally modern' prior to their dispersal to Australia. Using Wadley's (2001) 'symbolic storage' argument as a basis for identifying behavioural modernity in the Australian archaeological record, data on the temporal distribution of artefacts which could be classified as art, personal ornamentation, style in lithics, or the formal use of space were analysed. At the time of their analysis, Brumm and Moore (2005) found 149 Pleistocene sites, with only 16 of these exhibiting strong evidence for symbolic storage. Comparison of the rate of site establishment against the number of sites with evidence for symbolic storage in 10,000 year time-slices was presented, and it was argued that population density may be the main factor driving the observed pattern towards more instances of symbolic storage through time. They concluded that the evidence for symbolic storage, a key part of the evidence for behavioural modernity, was "patchy and that many of the hallmarks do not emerge until the middle to late Holocene" (Brumm and Moore, 2005:157). They argued that the evidence for symbolic behaviour in Pleistocene Australia more closely resembles the European Lower and Middle Palaeolithic than the Upper Palaeolithic because the evidence occurs in geographically and chronologically isolated patterns, coupled with ambiguity in the artefacts and features recovered. Having said this, Brumm and Moore (2005:161) conceded that "there can be little doubt that symbols were in use during early stages of the occupation of Australia”, as evidenced by the Mandu Mandu and Riwi shell beads, and that using Wadley's (2001) definition, these examples alone demonstrated the presence of behavioural modernity at this early period in the Australian archaeological record. Furthermore, they stated that "[w] hat appears to be 'patchy' evidence may be an illusion created by the destruction of the archaeological record through time" and that "the pace of spatial and chronological change may be biased in earlier periods because there are such huge gaps in the information database" (Brumm and Moore, 2005:168-169).

Along similar lines, Habgood and Franklin (2008) (also see Franklin and Habgood, 2007) examined the late Pleistocene archaeological record of Sahul to establish if the 'package' representing behavioural modernity as defined by the traditional trait lists was brought to Sahul by the earliest colonising groups. The analysis collated the evidence for behavioural modernity as defined by McBrearty and Brooks (2000) and plotted its temporal distribution. Habgood and Franklin (2008) found that the 'package' of behavioural modernity traits was not evident at the earliest sites, instead concluding that the components were gradually assembled over a 
30,000 year period following colonisation - likely as a product of increasing population density. Habgood and Franklin $(2008: 211,212)$ dismissed the possibility that taphonomy had significantly impacted the record, stating that "the presence of components of the "package" of traits at late Pleistocene sites in Greater Australia is not necessarily linked to the size of the excavation, amount of artefacts recovered, or the intensity of site usage in prehistoric times", and therefore, that the "patterning we have identified in the archaeologically visible traces of the "package" of modern human behaviour does not appear to be the result of taphonomic processes alone". Despite these statements, this study did not examine the effects of sampling and taphonomy on the Sahul archaeological record investigated.

In response, Langley et al. (2011) undertook a comprehensive review of behavioural modernity in Sahul through collating data from all Pleistocene archaeological sites known at that time. Langley et al. (2011) identified 128 instances of symbolic behaviour (ornamentation, pigment use, notational pieces, rock art, ritual burial, and intentional cranial deformation) from 89 sites. Examination of depositional context, presence or absence of indicative artefact categories, excavation size, and excavation methods found that taphonomy and sample size were significant factors in the patterning of Sahul's Pleistocene archaeological record. In particular, it was found that while adequate investigative methods had been used by the majority of researchers, the extreme environments, highly variable climates, over-representation of sites in poor preservational environments, and small average size of excavations restricted the recovery of evidence for complex behaviour dating to the earliest period of occupation. Increasing population density was also seen as a significant factor in the observed temporal and geographic patterning. Langley et al. (2011) concluded that there was no evidence to support Habgood and Franklin's (2008) supposition that Sahul lacked a number of the key 'traits' prior to 20,000 years ago.

The Pleistocene archaeological records of Sunda and Wallacea have long been either omitted or glossed over in global narratives of human symbolic development, though the startling discoveries of the past few years have begun to unravel the foundations originally laid down by Movius $(1944,1948)$ regarding the 'backward-ness' of the region. Indeed, maps plotting the distribution of Pleistocene-age art sites published just over 20 years ago only include one site in the entire region (e.g., Bednarik, 1994). More recently, researchers were still justifiably making statements such as "[n]onlithic modern behavioural traits such as tools made on bone, antler, and shell, as well as projectile points, figurative art, musical instruments, and personal ornaments are absent" (Pawlik, 2005:186-187). Thus, while many have been challenging the view that Sunda and Wallacea have little to offer global discussions of early symbolism or other forms of human complexities for the past two decades (e.g., Szabó et al., 2007; Huang et al., 2009; Gao, 2013; Langley et al., 2018), this campaign has only really gathered momentum in the past few years owing to new work in the region.

As can be seen by the preceding review, the temporal patterning of symbolic artefacts within Sahul's Pleistocene archaeological record is commonly explained in terms of increasing population density (Brumm and Moore, 2005; O'Connell and Allen, 2007; Habgood and Franklin, 2008; Balme et al., 2009; Langley et al., 2011), and/or taphonomic processes (Brumm and Moore, 2005; Davidson, 2007; Langley et al., 2011). And in Sunda and Wallacea, patterning has been non-existent until now. So, where does the evidence for symbolic behaviour in Sunda, Wallacea, and Sahul currently stand? 


\section{Current Evidence: Sahul}

Below we tabulate the archaeological evidence. Note that ages presented in the following Tables are those derived from the original publications, and issues relating to these ages are discussed within the main text. Where single ages are reported for evidence we have listed them with their laboratory identification number. Where instances are bracketed by ages, these brackets are listed.

Radiocarbon dates reported here were calibrated using OxCal (version 4.3) (Bronk Ramsey, 2009). Terrestrial radiocarbon ages were calibrated using INTCAL13 (Reimer et al., 2013) for sites further north than $-17.5^{\circ} \mathrm{S}$ and SHCal13 for sites located south of $-17.5^{\circ} \mathrm{S}$. The differentiation in use of the northern and southern hemisphere calibration datasets acknowledges the influence of the southward penetration of the Inter-Tropical Convergence Zone (Hogg et al., 2013; Hua et al., 2012). Marine radiocarbon ages were calibrated using MARINE13 (Reimer et al., 2013) with $\Delta$ R values after Ulm (2006) and Petchey and Ulm (2012). Radiocarbon ages with asymmetrical error estimates were calibrated using the larger error. Luminescence (OSL and TL) ages are presented in ka. OSL=optically stimulated luminescence; $\mathrm{U}=\mathrm{U}$-series; $\mathrm{AMS}=$ accelerator mass spectrometry radiocarbon dating; $\mathrm{LSC}=$ liquid scintillation counting (radiocarbon); $14 \mathrm{C}=$ radiocarbon dating where method could not be determined; $\mathrm{C}: \mathrm{R}=$ catio-ratio. 


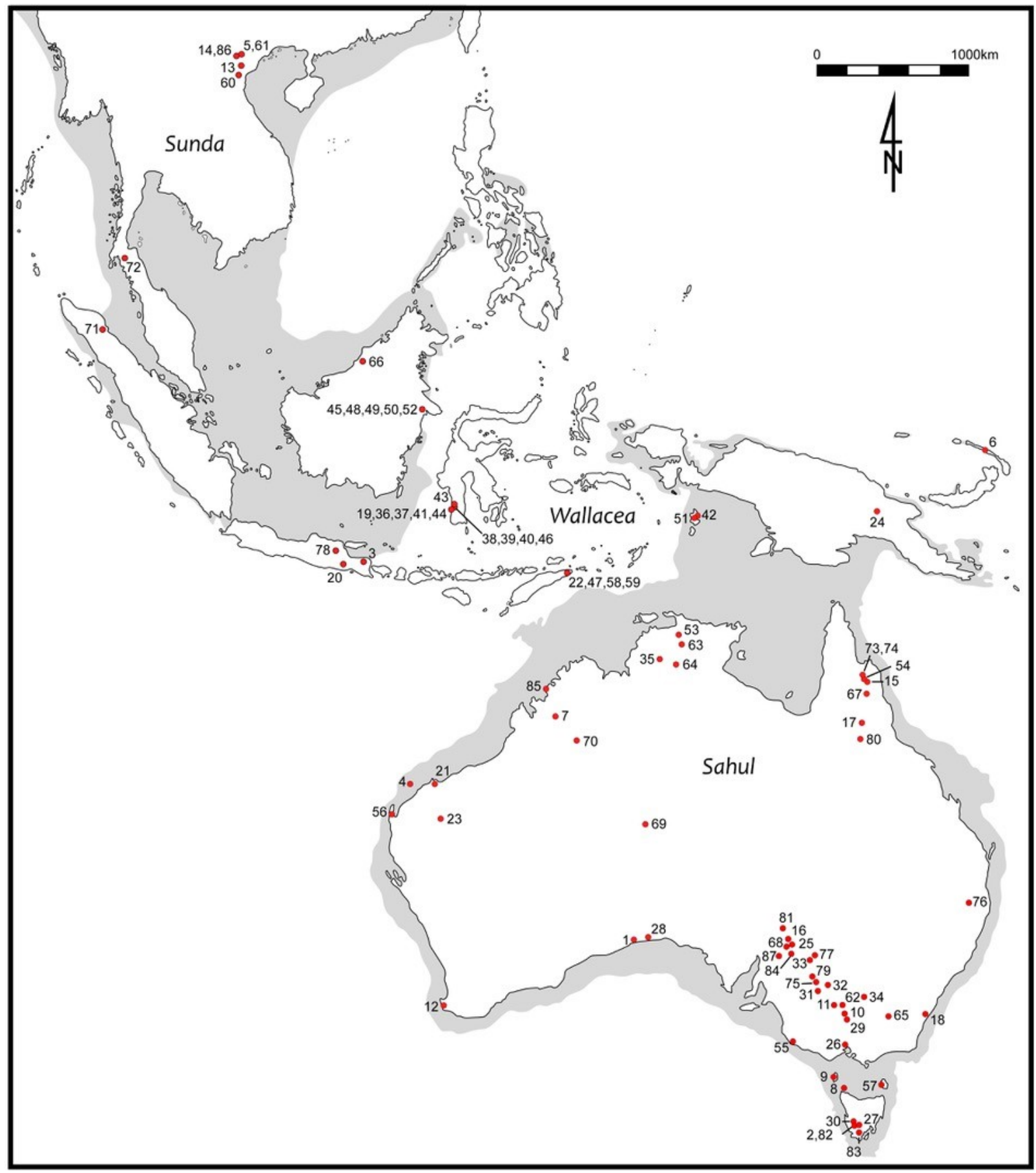


Figure 1. Location of Pleistocene sites with securely dated symbolic evidence. (1) Allen's Cave; (2) Ballawinne; (3) Betpuruh Cave; (4) Boodie Cave; (5) Buang Cave; (6) Buang Merabak; (7) Carpenter's Gap 1; (8) Cave Bay Cave; (9) Cliff Cave; (10) Cohuna; (11) Coobool Creek; (12) Devil's Lair; (13) Dong-Noi Cave; (14) Du Sang; (15) Early Man; (16) Eight Mile Creek; (17) Fern Cave; (18) Gnatalia Creek; (19) Gua Jing; (20) Gua Lawa; (21) Gum Tree Valley; (22) Jerimalai; (23) Juukan 2; (24) Kafiavana; (25) Karolta 1; (26) Keilor; (27) Keyhole Cavern; (28) Koonalda; (29) Kow Swamp; (30) Kutikina; (31) Lake Benanee; (32) Lake Mungo; (33) Lake Tandou; (34) Lake Urana; (35) Laurie Creek; (36) Leang Barugayya 1; (37) Leang Barugayya 2; (38) Leang Bulu Bettue; (39) Leang Burung 2; (40) Leang Jarie; (41) Leang Lompoa; (42) Leang Nabulei Lisa; (43) Leang Sakapao 1; (44) Leang Sampeang; (45) Liang Téwét; (46) Leang Timpuseng; (47) Lene Hara; (48) Liang Banteng; (49) Liang Karim; (50) Lubang Jeriji Saléh; (51) Liang Lemdubu; (52) Liang Sara; (53) Madjedbebe; (54) Magnificent Gallery; (55) Malangine; (56) Mandu Mandu; (57) Mannalargenna; (58) Matja Kuru 1; (59) Matja Kuru 2; (60) My-Tê Cave; (61) Na-Ca Cave; (62) Nacurrie; (63) Nauwalabila 1; (64) Nawarla Gabarnmang; (65) New Guinea II; (66) Niah Caves; (67) Nonda Rock; (68) Panaramitte North; (69) Puritjarra; (70) Riwi; (71) Saentis Midden; (72) Saikai Cave; (73) Sandy Creek 1; (74) Sandy Creek 2; (75) Snaggy Bend; (76) Talgai; (77) Tandou Creek; (78) Trinil; (79) Tuckers Creek; (80) Walkunder Arch Cave; (81) Warratyi; (82) Warreen; (83) Wargata Mina; (84) Wharton Hill; (85) Widgingarri 1; (86) Xom Trai; (87) Yunta Springs. Len-Dat Cave and Lam-Gan Cave not shown. 


\subsection{Colourants}

The decorative use of mineral-based pigments remains the earliest evidence for the practice of symbolic behaviours in Sahul. New excavations and analyses have significantly pushed back the dates of these instances. The presence of ochre in a deposit does not necessarily indicate use and some previous authors have included both utilised and unutilised pigments in previous syntheses (e.g., Flood 1995). In many cases, the presence of colourants are reported from a Pleistocene context, but no mention of whether they bear signs of use (facets, striations, flaking, etc.) are given. For example, at Nawamoyn, Schrire (1982) reports 247 pieces of ochre (red, yellow, white, orange, purple) dating up to 21,450 $\pm 380 \mathrm{BP}$ (ANU-51), but it is not stated whether they have evidence for use (see Dortch, 1986 for another example from Miriwun). Only instances of pigments reported to exhibit evidence for utilisation are included in Table 1.

Recent excavations at Madjedbebe (formally Malakunanja II) not only pushed back the initial arrival of people to Sahul to 65,000 years, but revealed extensive use of ground ochre throughout the sequence, with at least $3.5 \mathrm{~kg}$ of ground ochre found in the lowest dense layer (Phase 2) from five excavation squares spanning an occupation period between $65.0 \pm 5.7$ and $52.7 \pm 4.3 \mathrm{ka}$. Haematite was recovered below this dense occupation level (in Phase 1), which was dated by the excavators to $80.2 \pm 9.0$ to $71.0 \pm 7.3 \mathrm{ka}$ (Clarkson et al., 2017), though others have critiqued the chronology as presented (O'Connell et al., 2018; but see Clarkson et al., 2018 response). Use wear has been observed on many of these pieces, indicating a range of grinding substrates in extracting powder as well as potential direct application on a range of surfaces (Cox, 2013; Clarkson et al., 2017). Use wear and residue studies of grinding stones from Phase 2 of Madjedbebe also indicate extensive pigment production was carried out from the earliest occupation of the site. Additionally, cut sheet mica was found wrapped around an exotic yellow ochre crayon in Phase 2, indicating a strong association between reflective mica and mineral pigment beginning in the early occupation phase at the site (see 'Rock Art' below) (Clarkson et al., 2017).

Cases for the utilisation of red pigments next appear at Nauwalabila 1 where pieces perhaps dating back to 53,000 BP are stated as displaying grinding facets (Jones and Johnson, 1985; Roberts et al., 1993, 1994). At Carpenter's Gap 1, located to the southwest of Madjedbebe in the Kimberleys of Western Australia, a 20.5 cm-long piece of painted roof fall is bracketed by ages of 36,465 and 49,919 cal. BP (O'Connor and Fankhauser, 2001). Also found at this site, and described in more detail below, was a nose-bone $(>42,104$ cal. BP) exhibiting traces of red ochre on its surfaces, the distribution and quantity suggesting that the ornament was not painted, but accrued the ochre through incidental rubbing against a painted body. Thus, while indirect in its nature, this bone artefact gives an indication that body painting using at least red pigments has a long history on this continent (Langley et al., 2016a).

Similarly aged is the ochred burial of LM3 found on the other side of the continent at Willandra Lakes in New South Wales (NSW) (Allen, 1972; Bowler, 1998). After 40,000-years-ago, the number of instances of utilised ochre, ochre-stained artefacts, and ochred burials grows exponentially (Table 1). These finds do not show any spatial patterning in their appearance to indicate the movement of ideas or groups. 
Table 1: Current evidence for Pleistocene utilised colourants in Sahul. ACT=Australian Capital Territory; NSW=New South Wales; NG=New Guinea; NT=Northern Territory; $Q L D=Q u e e n s l a n d ;$ SA=South Australia; TAS=Tasmania; VIC=Victoria; WA=Western Australia.

\begin{tabular}{|c|c|c|c|}
\hline \multicolumn{4}{|l|}{ Utilised Ochre } \\
\hline Site & Age & Evidence & Source \\
\hline $\begin{array}{l}\text { Madjedbebe, } \\
\text { NT }\end{array}$ & $\begin{array}{l}\text { OSL: } 71.0 \pm 7.3 \text { to } 80.2 \pm 9.0 \mathrm{ka} \\
\text { (Phase } 1)^{*}\end{array}$ & Ground red ochre & $\begin{array}{l}\text { Clarkson et al., } \\
2015,2017\end{array}$ \\
\hline $\begin{array}{l}\text { Madjedbebe, } \\
\text { NT }\end{array}$ & $\begin{array}{l}\text { OSL: } 52.7 \pm 4.3 \text { to } 65.0 \pm 5.7 \mathrm{ka} \\
\text { (Phase } 2)^{*}\end{array}$ & $\begin{array}{l}\text { Ground red and yellow ochre, some wrapped in } \\
\text { cut sheet mica }\end{array}$ & $\begin{array}{l}\text { Clarkson et al., } \\
2015,2017\end{array}$ \\
\hline $\begin{array}{l}\text { Madjedbebe, } \\
\text { NT }\end{array}$ & $\begin{array}{l}\text { OSL: } 52.7 \pm 4.3 \text { to } 65.0 \pm 5.7 \mathrm{ka} \\
\text { (Phase 2) }\end{array}$ & $\begin{array}{l}\text { Grinding stones with ochre residue and use- } \\
\text { wear }\end{array}$ & $\begin{array}{l}\text { Clarkson et al., } \\
2015,2017\end{array}$ \\
\hline $\begin{array}{l}\text { Nauwalabila } 1, \\
\text { NT }\end{array}$ & $\begin{array}{l}\text { OSL: } 53.4 \pm 5.4 \mathrm{ka}\left(\mathrm{Ox}_{\mathrm{od}} \mathrm{K} 168\right) \\
\text { to } \\
\text { modern }\end{array}$ & $\begin{array}{l}\text { Red ochre and hematite - many pieces with } \\
\text { use/grinding facets in a wide variety of colours } \\
\text { found from this age and younger to the top of } \\
\text { the deposit }\end{array}$ & $\begin{array}{l}\text { Jones and } \\
\text { Johnson, 1985; } \\
\text { Roberts et al., } \\
\text { 1993, } 1994\end{array}$ \\
\hline $\begin{array}{l}\text { Madjedbebe, } \\
\text { NT }\end{array}$ & $\begin{array}{l}\text { OSL: } 28.1 \pm 2.8 \text { to } 51.6 \pm 4.2 \mathrm{ka} \\
\text { (Phase } 3 \text { ) }\end{array}$ & Ground red and yellow ochre & $\begin{array}{l}\text { Clarkson et al., } \\
\text { 2015, 2017; } \\
\text { Cox, } 2013\end{array}$ \\
\hline $\begin{array}{l}\text { Madjedbebe, } \\
\text { NT }\end{array}$ & $\begin{array}{l}\text { OSL: } 28.1 \pm 2.8 \text { to } 51.6 \pm 4.2 \mathrm{ka} \\
\text { (Phase 3) }\end{array}$ & $\begin{array}{l}\text { Painted sandstone slab coated in reflective } \\
\text { pigment (ochre mixed with ground mica) }\end{array}$ & $\begin{array}{l}\text { Clarkson et al., } \\
2017\end{array}$ \\
\hline $\begin{array}{l}\text { Nawarla } \\
\text { Gabarnmang }\end{array}$ & $\begin{array}{l}\text { AMS: } 39,488 \pm 809 \text { BP (Wk- } \\
34956) \\
2 \sigma: 42,212-44,809 \text { cal. BP } \\
\text { to } \\
\text { AMS: } 44,094 \pm 1882 \text { BP (Wk- } \\
34542 \text { ) } \\
2 \sigma:>45,087 \text { cal. BP-beyond } \\
\text { calibration curve }\end{array}$ & $\begin{array}{l}\text { Red ochre crayon with facetted edges and use- } \\
\text { striations. The older date is beyond the } \\
\text { available calibration curve. David et al. } \\
\text { (2019:74) assign a modelled age range of } \\
34,610-52,160 \text { cal BP for the artefact. }\end{array}$ & $\begin{array}{l}\text { David et al. } \\
2019\end{array}$ \\
\hline Warratyi, SA & $\begin{array}{l}\text { AMS: } 20,570 \pm 70 \mathrm{BP} \text { (Wk- } \\
37314) \\
2 \sigma: 24,406-25,042 \text { cal. BP } \\
\text { to } \\
\text { AMS: } 44,100 \pm 2200 \mathrm{BP}(\mathrm{Wk}- \\
39526) \\
2 \sigma:>44,741 \text { cal. BP - beyond } \\
\text { calibration curve }\end{array}$ & $\begin{array}{l}14 \text { pieces of red ochre reported but only one } \\
\text { sample recorded as worked, although the } \\
\text { provenance of this sample is not stated }\end{array}$ & $\begin{array}{l}\text { Hamm et al., } \\
2016\end{array}$ \\
\hline
\end{tabular}




\begin{tabular}{|c|c|c|c|}
\hline Warreen, TAS & 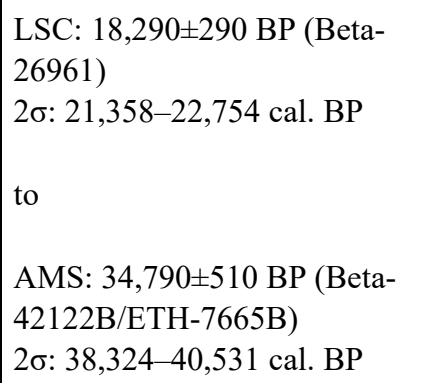 & $\begin{array}{l}\text { Ochre with facets and stained pieces of } \\
\text { limestone throughout deposit }\end{array}$ & $\begin{array}{l}\text { Allen, 1996; } \\
\text { Allen et al., } \\
\text { 1989; McGowan } \\
\text { et al., } 1993\end{array}$ \\
\hline Warratyi, SA & $\begin{array}{l}\text { AMS: } 29,240 \pm 280 \mathrm{BP}(\mathrm{Wk}- \\
41180) \\
2 \sigma: 32,729-33,941 \mathrm{cal} . \mathrm{BP} \\
\text { to } \\
\text { AMS: }>34,680 \pm 320 \mathrm{BP}(\mathrm{Wk}- \\
37317) \\
2 \sigma: 38,508-39,904 \text { cal. BP }\end{array}$ & $\begin{array}{l}\text { White spheroids (2-30 mm diameter) identified } \\
\text { as gypsum. Transported to site and assumed to } \\
\text { be used as a pigment for body paint }\end{array}$ & $\begin{array}{l}\text { Hamm et al., } \\
2016\end{array}$ \\
\hline Puritjarra, NT & $\begin{array}{l}\text { LSC: } \leq 32,400+500 \text { BP (ANU- } \\
7823) \\
2 \sigma: 35,643-36,950 \text { cal. BP }\end{array}$ & Ochre with facetted surfaces & $\begin{array}{l}\text { Rosenfeld, 1999; } \\
\text { Smith et al. } 1998\end{array}$ \\
\hline $\begin{array}{l}\text { Sandy Creek } \\
1, \text { QLD }\end{array}$ & $\begin{array}{l}\text { LSC: } 31,900+700 /-600 \mathrm{BP} \\
\text { (SUA-2870) } \\
2 \sigma: 34,609-37,784 \text { cal. BP }\end{array}$ & $\begin{array}{l}\text { Two pieces of striated red pigments; ochre } \\
\text { common throughout site }\end{array}$ & $\begin{array}{l}\text { Cole et al., 1995; } \\
\text { Morwood et } \\
\text { al.,1995; } \\
\text { Morwood and } \\
\text { Trezise, } 1989\end{array}$ \\
\hline $\begin{array}{l}\text { Fern Cave, } \\
\text { QLD }\end{array}$ & $\begin{array}{l}\text { AMS: } 30,300 \pm 800 \mathrm{BP}(\mathrm{OZD}- \\
205) \\
2 \sigma: 32,805-36,159 \mathrm{cal} . \mathrm{BP} \\
\text { to } \\
\text { modern }\end{array}$ & $\begin{array}{l}\text { Ochre with striations, possibly from } 3 \text { different } \\
\text { sources }\end{array}$ & $\begin{array}{l}\text { David, 1991; } \\
\text { David et al., } \\
1999\end{array}$ \\
\hline $\begin{array}{l}\text { Widgingarri } 1 \text {, } \\
\text { WA }\end{array}$ & $\begin{array}{l}\text { AMS: } 28,060 \pm 600 \mathrm{BP}(\mathrm{R}- \\
11795) \\
2 \sigma: 30,720-33,196 \mathrm{cal} . \mathrm{BP} \\
\text { to } \\
\text { modern }\end{array}$ & $\begin{array}{l}\text { Ochre in all levels - some with grinding facets } \\
\text { - red, white, yellow, orange. Faceted iron ore } \\
\text { pieces were also found, but their distribution is } \\
\text { not reported. }\end{array}$ & O’Connor, 1999 \\
\hline $\begin{array}{l}\text { Madjedbebe, } \\
\text { NT }\end{array}$ & $\begin{array}{l}\text { OSL: } 26.7 \pm 2.8-13.2 \pm 1.3 \mathrm{ka} \\
\text { (Phase } 4)\end{array}$ & Ground red and yellow ochre & $\begin{array}{l}\text { Clarkson et al., } \\
\text { 2015, } 2017\end{array}$ \\
\hline $\begin{array}{l}\text { Early Man, } \\
\text { QLD }\end{array}$ & $\begin{array}{l}\text { LSC: } 18,200 \pm 450 \mathrm{BP}(\mathrm{ANU}- \\
1565) \\
2 \sigma: 20,883-23,083 \mathrm{cal} . \mathrm{BP}\end{array}$ & Pigment - possibly some with striations & $\begin{array}{l}\text { David, 1991; } \\
\text { Rosenfeld et al., } \\
1981\end{array}$ \\
\hline
\end{tabular}




\begin{tabular}{|c|c|c|c|}
\hline $\begin{array}{l}\text { Nonda Rock, } \\
\text { QLD }\end{array}$ & $\begin{array}{l}\text { AMS: } 10,170 \pm 60 \mathrm{BP}(\mathrm{OZD} \\
874), \\
2 \sigma: 11,501-12,105 \mathrm{cal} . \mathrm{BP} \\
\text { to } \\
\text { AMS: } 11,300 \pm 60 \mathrm{BP}(\mathrm{OZD} \\
875) \\
2 \sigma: 13,064-13,275 \mathrm{cal} . \mathrm{BP}\end{array}$ & $\begin{array}{l}\text { Two pieces of ochre with striations and } \\
\text { beveling. A further } 18 \text { pieces of pigment do not } \\
\text { show evidence of use-wear. }\end{array}$ & $\begin{array}{l}\text { David et al., } \\
2007\end{array}$ \\
\hline $\begin{array}{l}\text { Magnificent } \\
\text { Gallery, QLD }\end{array}$ & $\begin{array}{l}\text { LSC: } 10,250 \pm 90 \text { BP (SUA- } \\
2876) \\
2 \sigma: 11,621-12,400 \text { cal. BP }\end{array}$ & $\begin{array}{l}3 \text { pieces of pigment }(6.8 \mathrm{~g}) \text { in spits } 34-38 \text { plus } \\
\text { continues to Holocene }-1 \text { piece striated red } \\
\text { pigment }\end{array}$ & $\begin{array}{l}\text { Morwood and } \\
\text { Jung, } 1995\end{array}$ \\
\hline \multicolumn{4}{|c|}{ Ochre-Stained Artefacts } \\
\hline Site & Age (cal. BP) & Evidence & Source \\
\hline Warratyi, SA & $\begin{array}{l}\text { AMS: }>25,900 \pm 180 \mathrm{BP}(\mathrm{Wk}- \\
40914) \\
2 \sigma: 29,553-30,631 \mathrm{cal} . \mathrm{BP} \\
\text { to } \\
\text { AMS: } 44,100 \pm 2200 \mathrm{BP}(\mathrm{Wk}- \\
39526) \\
2 \sigma:>44,741 \text { cal. BP - beyond } \\
\text { calibration curve }\end{array}$ & $\begin{array}{l}\text { Red ochre residues on } 2 \text { stone artefacts } \\
\text { (Artefact } \# 10 \text { and } \# 20 \text { ). Artefact } \# 20 \text { exhibited } \\
\text { ochre regularly inset from the left lateral } \\
\text { margin by c. } 1 \mathrm{~mm} \text { and concentrated adjacent to } \\
2 \text { large bending flake scars }\end{array}$ & $\begin{array}{l}\text { Hamm et al., } \\
2016\end{array}$ \\
\hline $\begin{array}{l}\text { Carpenter's } \\
\text { Gap 1, WA }\end{array}$ & $\begin{array}{l}\text { AMS: } 33,600 \pm 500 \mathrm{BP} \\
\text { (ANUA-7626) } \\
2 \sigma: 36,465-39,030 \mathrm{cal} . \mathrm{BP} \\
\text { to } \\
\text { AMS: } 42,800 \pm 1850 \mathrm{BP}(\mathrm{OZD}- \\
\text { 161) } \\
2 \sigma: 43,877-49,919 \mathrm{cal} . \mathrm{BP}\end{array}$ & $\begin{array}{l}\text { Limestone roof fall slab ( } 205 \mathrm{~mm} \times 75 \mathrm{~mm} \times \\
28 \mathrm{~mm} \text { ) with red painted pigment on surface. } \\
\text { Dates bracket age of slab. }\end{array}$ & $\begin{array}{l}\text { O'Connor and } \\
\text { Fankhauser, } \\
2001\end{array}$ \\
\hline $\begin{array}{l}\text { Carpenter's } \\
\text { Gap 1, WA }\end{array}$ & $\begin{array}{l}\text { AMS: }>40,100 \pm 1220 \text { BP } \\
(\text { ANU-ABOx340) } \\
2 \sigma:>42,104-46,294 \text { cal. BP }\end{array}$ & $\begin{array}{l}\text { Worked kangaroo fibulae ('nose-bone') with } \\
\text { red ochre stains }\end{array}$ & $\begin{array}{l}\text { Langley et al., } \\
2016 \mathrm{a}\end{array}$ \\
\hline $\begin{array}{l}\text { Madjedbebe, } \\
\text { NT }\end{array}$ & $\begin{array}{l}\text { OSL: } 26.7 \pm 2.8-13.2 \pm 1.3 \mathrm{ka} \\
\text { (Phase } 4 \text { ) }\end{array}$ & Ochre stained grindstone & $\begin{array}{l}\text { Clarkson et al., } \\
2015,2017\end{array}$ \\
\hline $\begin{array}{l}\text { Mannalargenn } \\
\text { a, TAS }\end{array}$ & $\begin{array}{l}\text { LSC:>10,430 } \pm 90 \text { BP (ANU- } \\
9001) \\
2 \sigma:>12,005-12,580 \mathrm{cal} . \mathrm{BP} \\
\text { to } \\
\text { AMS: } 23,015 \pm 210 \mathrm{BP}(\mathrm{AA}- \\
13040) \\
2 \sigma: 26,879-27,704 \mathrm{cal} . \mathrm{BP}\end{array}$ & $\begin{array}{l}7 \text { quartz artefacts, none retouched or with use- } \\
\text { wear, all had reddish stains consistent with } \\
\text { ochre staining }\end{array}$ & Sim, 1998 \\
\hline
\end{tabular}




\begin{tabular}{|c|c|c|c|}
\hline $\begin{array}{l}\text { Cave Bay } \\
\text { Cave, TAS }\end{array}$ & $\begin{array}{l}\text { LSC: } 22,750 \pm 420 \text { BP (ANU- } \\
1498) \\
2 \sigma: 26,127-27,686 \text { cal. BP }\end{array}$ & 3 pieces of quartz with ochre adhering & Bowdler, 1984 \\
\hline $\begin{array}{l}\text { Mannalargenn } \\
\text { a, TAS }\end{array}$ & $\begin{array}{l}\text { LSC: }<18,400 \pm 200 \text { BP (SUA- } \\
2897) \\
2 \sigma:<21,981-22,419 \text { cal. BP }\end{array}$ & $\begin{array}{l}\text { Red colouring tentatively identified as ochre on } \\
2 \text { artefacts, a shell flaked piece and shell round- } \\
\text { edge scraper }\end{array}$ & Brown, 1993 \\
\hline Kafiavana, NG & $\begin{array}{l}\text { LSC: } \geq 10,730 \pm 370 \text { BP (ANU- } \\
41 b) \\
2 \sigma: 12,129-13,040 \text { cal. BP }\end{array}$ & 4 stones with adhering ochre & White, 1972 \\
\hline \multicolumn{4}{|l|}{ Ochred Burials } \\
\hline Site & Age & Evidence & Source \\
\hline $\begin{array}{l}\text { Lake Mungo, } \\
\text { NSW }\end{array}$ & $\begin{array}{l}\text { OSL: } 42.5 \pm 2.4 \mathrm{ka}(\mathrm{M} 1 \mathrm{R} \\
0.37 \mathrm{~m} / \mathrm{a}) \\
\text { to } \\
\text { OSL: } 46.6 \pm 2.3 \mathrm{ka}(\mathrm{M} 1 \mathrm{R} 0.0 \mathrm{~m})\end{array}$ & LM1 — cremation with ochre associated & $\begin{array}{l}\text { Bowler et al., } \\
\text { 1970; Bowler et } \\
\text { al., } 2003\end{array}$ \\
\hline $\begin{array}{l}\text { Lake Mungo, } \\
\text { NSW }\end{array}$ & $\begin{array}{l}\text { OSL: } 38.2 \pm 1.3 \mathrm{ka}(\mathrm{M} 3 \mathrm{~T} 61.2 \\
\mathrm{m}) \\
\text { to } \\
\text { OSL: } 42.2 \pm 2.5 \mathrm{ka}(\mathrm{M} 3 \mathrm{~T} 58.0 \\
\text { m) }\end{array}$ & LM3 - extended burial covered with ochre & $\begin{array}{l}\text { Bowler et al., } \\
\text { 2003; Thorne et } \\
\text { al., } 1999\end{array}$ \\
\hline $\begin{array}{l}\text { Kow Swamp, } \\
\text { VIC }\end{array}$ & $\begin{array}{l}\text { OSL: } 14.4 \pm 0.8 \mathrm{ka}(\mathrm{RP} 1) \\
\text { to } \\
\text { OSL: } 26 \pm 2 \mathrm{ka}(\mathrm{RP} 4)\end{array}$ & $\begin{array}{l}\text { Cemetery with }>40 \text { individuals buried with } \\
\text { grave goods including mussel shells, lithics, } \\
\text { marsupial teeth and ochre, kangaroo incisor } \\
\text { headband; infants and juveniles; at least } 1 \text { is } \\
\text { cremated }\end{array}$ & $\begin{array}{l}\text { Bowdler, 1993; } \\
\text { Pardoe, 1993a; } \\
\text { Stone and } \\
\text { Cupper, } 2003\end{array}$ \\
\hline $\begin{array}{l}\text { Cliff Cave, } \\
\text { TAS }\end{array}$ & $\begin{array}{l}\text { LSC: } 14,270 \pm 640 \text { BP (ANU- } \\
7039) \\
2 \sigma: 15,665-18,931 \text { cal. BP }\end{array}$ & $\begin{array}{l}\text { Red ochre found on cranium of buried } \\
\text { individual. Small pieces of red ochre were also } \\
\text { found on the cranium and femur suggesting the } \\
\text { body had ochre rubbed into it or that ochre was } \\
\text { placed with the bones }\end{array}$ & $\begin{array}{l}\text { Sim and Thorne, } \\
1990\end{array}$ \\
\hline
\end{tabular}




\subsection{Ornamentation}

Another recent discovery, the >42,104-46,294 cal BP 'nose-bone' from Carpenter's Gap 1 mentioned above, was made on kangaroo fibulae. This $12.9 \mathrm{~cm}$ long implement exhibits microscopic traces for shaping at the distal and proximal extremities in the form of grinding and scraping. Traces of a red colourant was also observed at both extremities. Comparison of the types, densities, and distribution of these manufacturing traces, use-wear, and residues with ethnographically collected items with known functions found that those recorded as 'nose-bones' were the closest match (Langley et al., 2016a). This artefact constitutes the oldest example of a personal ornament thus far found in a Sahul, Sunda, or Wallacean context, and is a type of facial ornamentation that has continued to be made and used to the present-day. As far as we are aware, this artefact also constitutes the oldest example of an ornament made on bone.

Recent direct dating of the 10 marine Dentalium sp. shell beads found at Riwi — and which were for a long time held to be the oldest bead evidence in Sahul (Balme, 2000) - has found that they are much younger than originally thought, redated from c.39,174 cal. BP (based on their stratigraphic context) to around 78808298 cal. BP (Balme et al., 2018). This reduction in age, now places them comfortably with other directly dated Dentalium beads from the nearby sites of Windjana Gorge 1 (examples dated to c.8,570 cal. BP) and Carpenter's Gap 3 (c.7,365 cal. BP) (Balme et al., 2018), though Boodie Cave on Barrow Island, has produced examples directly dated to 12,000 BP (Veth et al., 2017). Twenty-two shell beads made on Conus sp. from Mandu Mandu Creek Rockshelter dated to 31,592-40,745 cal. BP have now taken the place of the oldest beads (a small piece of perforated material that is threaded with others to make a necklace (etc.) or sewn onto fabric) in Sahul (Balme and Morse, 2006; Morse, 1993). In all instances, the marine shells displayed evidence for modification and use wear, and in the case of the younger Riwi beads, evidence for ochre and fibre fragments were also observed (Balme et al., 2018).

Continuing with marine-sourced raw materials, in the very north of Sahul a number of shell fragments and shark teeth originating from varying species are suggested to have been used as personal ornaments. These include a drilled tiger shark (Galeocerdo cuvier) tooth with a c. $2 \mathrm{~mm}$ perforation with evidence for bifacial drilling dated to $\leq 41,829-43,457$ cal. BP, along with a drilled Turbo sp. shell dated to 33,949-37,777 cal. BP, were found in Buang Merabak, New Britain (Leavesley, 2007; Leavesley and Allen, 1998). Similarly, at Kafiavana in Papua New Guinea three perforated Money cowry (Monetaria moneta) shells dating to 12,12913,040 cal. BP were recovered (White, 1972). On mainland Australia, fragments of marine shell far from the coast have commonly been suggested to have some symbolic value, probably as ornamentation (Smith and Veth, 2004). Examples include those recovered from Allen's Cave dated to around 14,000-13,000 BP (Cane, 2001).

At Devil's Lair in the southwest, three beads made from macropod fibulae dating to 13,576-23,103 and $13,576-13,931 \mathrm{cal}$. BP and a marl fragment with a $6.5 \mathrm{~mm}$ perforation dating to $13,462-23,493 \mathrm{cal}$. BP are reported (Dortch, 1979a, 1979b, 1980). The bone beads are unique to Devil's Lair, osseous beads or pendants of any type next appearing only in the early-to-mid Holocene (as at Roonka, Lake Nitchie, Bunyan, Kow Swamp; see Macintosh et al., 1970, Thorne and Macumber, 1972, Pretty, 1977, Feary, 1996). 
Table 2: Current evidence for the Pleistocene ornaments in Sahul.

\begin{tabular}{|c|c|c|c|}
\hline \multicolumn{4}{|c|}{ Osseous Ornaments } \\
\hline Site & Age & Evidence & Source \\
\hline $\begin{array}{l}\text { Carpenter's Gap } \\
1, \text { WA }\end{array}$ & $\begin{array}{l}>\text { AMS: } 40,100 \pm 1220 \mathrm{BP} \\
(\mathrm{ANU}-\mathrm{ABO} 340) \\
2 \sigma: 42,104-46,294 \mathrm{cal} . \\
\text { BP }\end{array}$ & $\begin{array}{l}\text { Worked kangaroo fibulae ('nose-bone') with red ochre } \\
\text { stains }\end{array}$ & $\begin{array}{l}\text { Langley et al., } \\
2016 \mathrm{a}\end{array}$ \\
\hline $\begin{array}{l}\text { Buang Merabak, } \\
\text { NG }\end{array}$ & $\begin{array}{l}\text { AMS: } \leq 39,090 \pm 550 \mathrm{BP} \\
\text { (ANUA-15808) } \\
2 \sigma: 41,829-43,457 \mathrm{cal} . \\
\text { BP }\end{array}$ & $\begin{array}{l}1 \text { perforated ( } 2 \mathrm{~mm} \text { diameter hole) (bifacially drilled) } \\
\text { tiger shark (Galeocerdo cuvier) tooth }\end{array}$ & $\begin{array}{l}\text { Leavesley, 2007; } \\
\text { Leavesley et al., } \\
2002\end{array}$ \\
\hline Juukan 2 & $\begin{array}{l}\text { AMS: } 24,410 \pm 110 \mathrm{BP} \\
\text { (Beta-383965) } \\
2 \sigma: 28,095-28,707 \mathrm{cal} \\
\text { BP. }\end{array}$ & $\begin{array}{l}\text { Sharp unipoint bone point made on kangaroo fibula } \\
\text { with horizontal striations, scratching and possible } \\
\text { pigment }\end{array}$ & Reynen, 2018 \\
\hline $\begin{array}{l}\text { Devil's Lair, } \\
\text { WA }\end{array}$ & 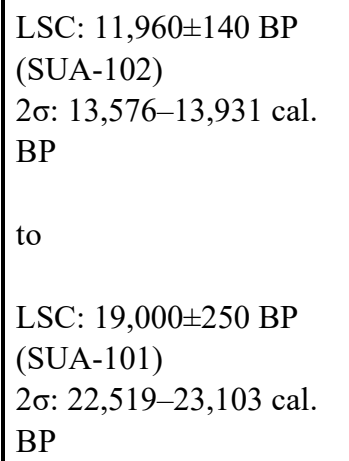 & $\begin{array}{l}2 \text { beads (B3654; B1898) and } 1 \text { ornament (A22028) } \\
\text { made on segments of macropod bone }\end{array}$ & $\begin{array}{l}\text { Dortch, 1979a, } \\
1979 b\end{array}$ \\
\hline $\begin{array}{l}\text { Kow Swamp, } \\
\text { VIC }\end{array}$ & $\begin{array}{l}\text { OSL: } 14.4 \pm 0.8 \mathrm{ka}(\mathrm{RP} 1) \\
\text { to } \\
\text { OSL: } 26 \pm 2 \mathrm{ka}(\mathrm{RP} 4)\end{array}$ & $\begin{array}{l}\text { Headband of kangaroo incisors with traces of resin } \\
\text { found in an ochred burial (Burial 16) }\end{array}$ & $\begin{array}{l}\text { Stone and } \\
\text { Cupper, } 2003\end{array}$ \\
\hline $\begin{array}{l}\text { Devil's Lair, } \\
\text { WA }\end{array}$ & $\begin{array}{l}\text { LSC: c. } 11,960 \pm 140 \mathrm{BP} \\
\text { (SUA-102) } \\
2 \sigma: 13,576-13,931 \mathrm{cal} . \\
\text { BP }\end{array}$ & 1 tube bead (B1556) made on macropod long bone & $\begin{array}{l}\text { Dortch, 1979a, } \\
1979 b\end{array}$ \\
\hline \multicolumn{4}{|c|}{ Marine Shell Ornaments } \\
\hline $\begin{array}{l}\text { Buang Merabak, } \\
\text { NG }\end{array}$ & $\begin{array}{l}\text { LSC: }<31,990 \pm 830 \mathrm{BP} \\
(\mathrm{ANU}-6614) \\
2 \sigma: 33,949-37,777 \mathrm{cal} . \\
\text { BP }\end{array}$ & 1 perforated (drilled) Turbo sp. shell & $\begin{array}{l}\text { Leavesley and } \\
\text { Allen, } 1998\end{array}$ \\
\hline
\end{tabular}




\begin{tabular}{|c|c|c|c|}
\hline $\begin{array}{l}\text { Mandu Mandu, } \\
\text { WA }\end{array}$ & 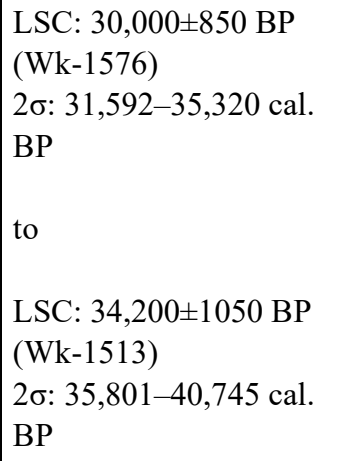 & $\begin{array}{l}22 \text { Conus sp. shell beads (whole and fragments) with } \\
\text { perforations and use-wear }\end{array}$ & $\begin{array}{l}\text { Morse, 1993; } \\
\text { Balme and } \\
\text { Morse, } 2006\end{array}$ \\
\hline $\begin{array}{l}\text { Allen's Cave, } \\
\text { SA }\end{array}$ & c. $14,000-$ c. $13,000 \mathrm{BP}$ & 1 Haliotis laevigata fragment - possibly an ornament & Cane, 2001 \\
\hline Kafiavana, NG & $\begin{array}{l}\text { LSC: } \geq 10,730 \pm 370 \mathrm{BP} \\
(\mathrm{ANU}-41 \mathrm{~b}) \\
2 \sigma: 12,129-13,040 \mathrm{cal} . \\
\mathrm{BP}\end{array}$ & 3 perforated money cowry (Monetaria moneta) shells & White, 1972 \\
\hline $\begin{array}{l}\text { Boodie Cave, } \\
\text { WA }\end{array}$ & 12,000 cal. BP & $\begin{array}{l}22 \text { fragments of Dentalium sp. shell beads with edge } \\
\text { notching and wear }\end{array}$ & Veth et al., 2017 \\
\hline $\begin{array}{l}\text { Liang Nabulei } \\
\text { Lisa, Aru }\end{array}$ & $\begin{array}{l}\text { AMS: } \geq 9630 \pm 60 \mathrm{BP} \\
\text { (OZD-697) } \\
2 \sigma: 10,767-11,186 \mathrm{cal} . \\
\mathrm{BP}\end{array}$ & $\begin{array}{l}\text { Terebra subulata shell with drilled perforation. May be } \\
\text { associated with the secondary burial of a child. }\end{array}$ & $\begin{array}{l}\text { O'Connor et al., } \\
2005 \mathrm{a}\end{array}$ \\
\hline \multicolumn{4}{|c|}{ Stone Ornaments } \\
\hline $\begin{array}{l}\text { Devil's Lair, } \\
\text { WA }\end{array}$ & 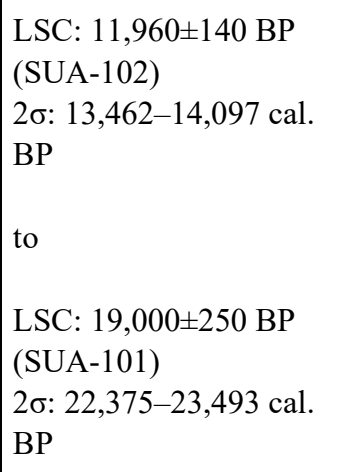 & Marl with single perforation - possibly an ornament & Dortch, 1980 \\
\hline \multicolumn{4}{|c|}{ Body Modification } \\
\hline $\begin{array}{l}\text { Kow Swamp, } \\
\text { VIC }\end{array}$ & $\begin{array}{l}\text { OSL: } 14.4 \pm 0.8 \mathrm{ka}(\mathrm{RP} 1) \\
\text { to } \\
\text { OSL: } 26 \pm 2 \mathrm{ka}(\mathrm{RP} 4)\end{array}$ & Individual (KS7) with intentional cranial deformation & $\begin{array}{l}\text { Antón and } \\
\text { Weinstein, 1999; } \\
\text { Stone and } \\
\text { Cupper, } 2003\end{array}$ \\
\hline $\begin{array}{l}\text { Coobool Creek, } \\
\text { NSW }\end{array}$ & $\begin{array}{l}\mathrm{U}: 14,300 \pm 1000 \mathrm{BP} \\
(\mathrm{LLO}-416)\end{array}$ & $\begin{array}{l}\text { Individual (Coobool Creek 65) with intentional cranial } \\
\text { deformation }\end{array}$ & Brown, 1989 \\
\hline $\begin{array}{l}\text { Kow Swamp, } \\
\text { VIC }\end{array}$ & $\begin{array}{l}\text { LSC: } 13,000 \pm 280 \text { (ANU- } \\
1236) \\
2 \sigma: 14,389-16,310 \mathrm{cal} . \\
\text { BP }\end{array}$ & Individual (KS5) with intentional cranial deformation & $\begin{array}{l}\text { Antón and } \\
\text { Weinstein, } 1999\end{array}$ \\
\hline
\end{tabular}




\begin{tabular}{|c|c|c|c|}
\hline Nacurrie, NSW & $\begin{array}{l}\text { LSC: } 11,440 \pm 160 \mathrm{BP} \\
\text { (not available) } \\
2 \sigma: 12,926-13,569 \mathrm{cal} . \\
\text { BP }\end{array}$ & Individual (N1) with intentional cranial deformation & $\begin{array}{l}\text { Brown, 1989; } \\
\text { Pardoe, 1993a }\end{array}$ \\
\hline $\begin{array}{l}\text { Kow Swamp, } \\
\text { VIC }\end{array}$ & $\begin{array}{l}\text { LSC: } 10,070 \pm 250 \mathrm{BP} \\
\text { (ANU-403b) } \\
2 \sigma: 10,791-12,544 \mathrm{cal} . \\
\text { BP }\end{array}$ & Individual (KS1) with intentional cranial deformation & $\begin{array}{l}\text { Antón and } \\
\text { Weinstein, } 1999\end{array}$ \\
\hline
\end{tabular}

In Sahul, decoration of the body was not restricted to the beading, painting, or piercing of the skin, but also the intentional deformation of the cranium. Three cases of this type of body modification were found at Kow Swamp, Victoria: KS5 dating to 14,389-16,310 cal. BP, KS1 dating to 10,791-12,544 cal. BP and KS7 dated only to the Pleistocene (Antón and Weinstein, 1999), but are likely to date to between $14.4 \pm 0.8 \mathrm{ka}$ and $26 \pm 2 \mathrm{ka}$ (Stone and Cupper, 2003). Another found at Coobool Creek 65 is associated with a general site Useries date of 14,300 \pm 1000 BP (LLO-416) (Brown, 1989). Finally, Nacurrie 1 is dated to 12,926-13,569 cal. BP (Brown, 1989, Pardoe, 1993a). Antón and Weinstein (1999) concluded that the Kow Swamp, Coobool Creek, and Nacurrie crania were all intentionally deformed by methods such as head binding or the placing of the mother's hands on an infant's skull over a period of time, and were not the result of taphonomic processes. All are found in the southeast of Sahul indicating that this behaviour may be a regionally and temporally confined cultural practice, though caution must be used in making this claim as skeletal remains are not found in great number outside of this region.

\subsection{Rock Art}

The extensive displays of pictographs and petroglyphs found throughout one of the areas where people are thought to have first entered Sahul (Arnhem Land in the Northern Territory and the Kimberley, WA) remain poorly dated despite extensive efforts to determine its age (Chippindale and Taçon, 1998, Aubert, 2012, David et al., 2013a, Mulvaney, 2013). The oldest direct evidence for rock art in Sahul remains the piece of painted roof fall recovered from Carpenter's Gap 1 in the Kimberley securely dated to between 36,465 and 49,919 cal. BP (O'Connor and Fankhauser, 2001), though new work at Madjedbebe (also in Arnhem Land) produced an ochre-covered slab dating somewhere between 51.6 \pm 4.2 and $28.1 \pm 2.8 \mathrm{ka}$, this artefact being the oldest known use of reflective paint comprised of red ochre mixed with ground reflective mica (Clarkson et al., 2017). A fragment of roof fall found at Nawarla Gabarnmang dated to 26,760-27,667 cal. BP is argued to represent the oldest piece of figurative art in Sahul (David et al., 2013b), However, we would argue that depictions of animal tracks should also be considered figurative art as they are an accurate and recognisable representation of the natural world. 
Table 3: Current evidence for Pleistocene rock art in Sahul. Note that ages presented in tables are those derived from the original publications. *See text for discussion of issues with ages from particular sites.

\begin{tabular}{|c|c|c|c|}
\hline \multicolumn{4}{|c|}{ Rock Art - Evidence Found in Archaeological Deposit } \\
\hline Site & Age & Evidence & Source \\
\hline $\begin{array}{l}\text { Carpenter's Gap } \\
1 \text {, WA }\end{array}$ & 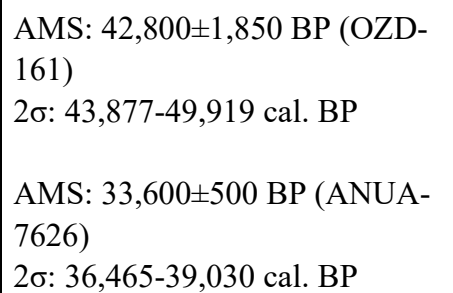 & $\begin{array}{l}\text { Limestone roof fall slab }(205 \mathrm{~mm} \times 75 \mathrm{~mm} \times \\
28 \mathrm{~mm} \text { ) with red painted pigment on surface. } \\
\text { Dates bracket age of slab. }\end{array}$ & $\begin{array}{l}\text { O'Connor and } \\
\text { Fankhauser, } \\
2001\end{array}$ \\
\hline Madjedbebe, NT & $\begin{array}{l}\text { OSL: } 51.6 \pm 4.2-28.1 \pm 2.8 \mathrm{ka} \\
\text { (Phase 3) }\end{array}$ & Conjoining ochre-covered slab & $\begin{array}{l}\text { Clarkson et al., } \\
2017\end{array}$ \\
\hline $\begin{array}{l}\text { Sandy Creek 2, } \\
\text { QLD }\end{array}$ & $\begin{array}{l}\text { AMS: } 24,600 \pm 220 \text { BP (NZA- } \\
2559) \\
2 \sigma: 28,111-29,155 \text { cal. BP }\end{array}$ & Pigment art on rockshelter wall below deposit & Cole et al., 1995 \\
\hline $\begin{array}{l}\text { Nawarla } \\
\text { Gabarnmang, } \\
\text { NT }\end{array}$ & $\begin{array}{l}\text { AMS: } 22,965 \pm 218 \text { BP (Wk- } \\
32840) \\
2 \sigma: 26,760-27,667 \text { cal BP }\end{array}$ & $\begin{array}{l}\text { Painted roof fall found in deposit. Set of lines } \\
\text { crossed by infilled curvilinear line. }\end{array}$ & $\begin{array}{l}\text { David et al., } \\
\text { 2013a }\end{array}$ \\
\hline $\begin{array}{l}\text { New Guinea II, } \\
\text { VIC }\end{array}$ & $\begin{array}{l}\text { LSC: } 4660 \pm 110 \text { BP (SUA-2217) } \\
2 \sigma: 4975-5588 \text { cal. BP } \\
\text { to } \\
\text { LSC: } 21,000+900 /-800 \text { (SUA- } \\
2222 \text { ) } \\
2 \sigma: 23,447-27,211 \text { cal. BP }\end{array}$ & $\begin{array}{l}\text { Figure flutings occurring in panels with multi- } \\
\text { directional marks and superimposed marks. } \\
\text { Dates for archaeological deposit at site. }\end{array}$ & Ossa et al., 1995 \\
\hline Madjedbebe, NT & $\begin{array}{l}\text { OSL: } 26.7 \pm 2.8-13.2 \pm 1.3 \mathrm{ka} \\
\text { (Phase 4) }\end{array}$ & $\begin{array}{l}\text { Mortar with possible outline motif, and } \\
\text { charcoal lines and dots on sandstone piece }\end{array}$ & $\begin{array}{l}\text { Clarkson et al., } \\
2017\end{array}$ \\
\hline Koonalda, SA & 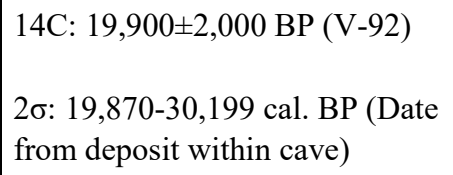 & $\begin{array}{l}74 \text { diagonal incised lines below } 37 \text { finger } \\
\text { flutings on limestone wall }\end{array}$ & $\begin{array}{l}\text { Bednarik, 1986; } \\
\text { Morwood, 1978, } \\
\text { 1982; Wright, } \\
1971\end{array}$ \\
\hline Koonalda, SA & $\begin{array}{l}\text { AMS: } 18,123 \pm 59 \text { BP }(\mathrm{Wk}- \\
35957) \\
2 \sigma: 21,675-22,162 \text { cal. BP }\end{array}$ & $\begin{array}{l}\text { Finger-fluted incised limestone slab found in } \\
\text { deposit. This is a minimum age (see Wright, } \\
\text { 1971) }\end{array}$ & Walshe 2017 \\
\hline $\begin{array}{l}\text { Early Man, } \\
\text { QLD }\end{array}$ & $\begin{array}{l}\text { LSC: } 13,200 \pm 170 \text { BP (ANU- } \\
1441) \\
2 \sigma: 15,605-16,118 \text { cal. BP }\end{array}$ & $\begin{array}{l}\text { Geometric and track motif petroglyphs on } \\
\text { shelter wall covered by archaeological deposit }\end{array}$ & $\begin{array}{l}\text { Rosenfeld et al. } \\
1981 ; \text { Rosenfeld, } \\
1993\end{array}$ \\
\hline $\begin{array}{l}\text { Sandy Creek 1, } \\
\text { QLD }\end{array}$ & $\begin{array}{l}\text { 14C: } 12,620 \pm 370 \text { BP (Beta- } \\
51089) \\
2 \sigma: 13,775-16,026 \text { cal. BP }\end{array}$ & Sandstone wall fall fragment with pecked motif & $\begin{array}{l}\text { Morwood et al., } \\
1995\end{array}$ \\
\hline
\end{tabular}




\begin{tabular}{|c|c|c|c|}
\hline \multicolumn{4}{|c|}{ Rock Art - Directly Dated } \\
\hline Karolta $1, \mathrm{SA}^{*}$ & $\mathrm{C}: \mathrm{R}: 44,760 \pm 3390 \mathrm{BP}$ & Pecked circle & Watchman, 1992 \\
\hline Karolta $1, \mathrm{SA}^{*}$ & $\mathrm{C}: \mathrm{R}: 44,590 \pm 1530 \mathrm{BP}$ & Pecked macropod tracks & Watchman, 1992 \\
\hline $\begin{array}{l}\text { Panaramittee } \\
\text { North, SA* }\end{array}$ & $\begin{array}{l}\text { AMS: } \geq 43,140 \pm 3000 \mathrm{BP}(\mathrm{AA}- \\
6898) \\
2 \sigma: 43,380-\text { beyond calibration } \\
\text { curve }\end{array}$ & Pounded and pecked curved line (PN6) & $\begin{array}{l}\text { Nobbs and Dorn, } \\
1993\end{array}$ \\
\hline $\begin{array}{l}\text { Wharton Hill, } \\
\text { SA* }\end{array}$ & $\begin{array}{l}\text { AMS: } \geq 35,530 \pm 650 \text { BP (NZA- } \\
2361) \\
2 \sigma: 38,773-41,450 \mathrm{cal} . \mathrm{BP}\end{array}$ & Pounded and pecked oval (WH5) & $\begin{array}{l}\text { Dorn and Nobbs, } \\
\text { 1992; Nobbs and } \\
\text { Dorn, } 1993\end{array}$ \\
\hline Karolta $1, \mathrm{SA}^{*}$ & $\begin{array}{l}\text { AMS: } \geq 31,230 \pm 920 \text { BP (NZA- } \\
1370) \\
2 \sigma: 33,683-37,807 \text { cal. BP }\end{array}$ & $\begin{array}{l}\text { Pounded and pecked curved line superimposed } \\
\text { by motif (K26) }\end{array}$ & $\begin{array}{l}\text { Dorn and Nobbs, } \\
\text { 1992; Nobbs and } \\
\text { Dorn, } 1993\end{array}$ \\
\hline Karolta $1, \mathrm{SA}^{*}$ & $\begin{array}{l}\text { AMS: } \geq 30,230 \pm 770 \text { BP (NZA- } \\
1378) \\
2 \sigma: 32,794-36,018 \mathrm{cal} . \mathrm{BP}\end{array}$ & Pounded and pecked curved line (K23) & $\begin{array}{l}\text { Dorn and Nobbs, } \\
\text { 1992; Nobbs and } \\
\text { Dorn, } 1993\end{array}$ \\
\hline Gnatalia Creek & $\begin{array}{l}\text { AMS: } 29,795 \pm 420 \text { BP (AA- } \\
5851) \\
2 \sigma: 33,066-34,675 \text { cal BP }\end{array}$ & $\begin{array}{l}\text { Red and black large curvilinear non-figurative } \\
\text { drawing. }\end{array}$ & $\begin{array}{l}\text { McDonald et al., } \\
1990\end{array}$ \\
\hline $\begin{array}{l}\text { Walkunder Arch } \\
\text { Cave, QLD }\end{array}$ & $\begin{array}{l}\text { AMS: } 28,100 \pm 400 \text { BP (OZA- } \\
391) \\
2 \sigma: 31,184-33,052 \text { cal. BP }\end{array}$ & Red pigment on rock wall & $\begin{array}{l}\text { Campbell et al., } \\
1996\end{array}$ \\
\hline Malangine, SA & $\mathrm{U}:<28,000 \pm 2000 \mathrm{BP}$ & Possible finger flutings & Bednarik, 1999 \\
\hline $\begin{array}{l}\text { Walkunder Arch } \\
\text { Cave, QLD }\end{array}$ & $\begin{array}{l}\text { AMS: } 25,800 \pm 280(\text { OZA-392) } \\
2 \sigma: 29,300-30,702 \text { cal. BP } \\
\text { to } \\
\text { AMS: } 22,800 \pm 210 \mathrm{BP}(\mathrm{OZA}- \\
393) \\
2 \sigma: 26,545-27,486 \mathrm{cal} . \mathrm{BP}\end{array}$ & Yellow goethite paint on rock wall & $\begin{array}{l}\text { Campbell et al., } \\
1996\end{array}$ \\
\hline Karolta $1, \mathrm{SA}^{*}$ & $\begin{array}{l}\text { AMS: } \geq 22,480 \pm 340 \text { BP (NZA- } \\
1366) \\
2 \sigma: 26,044-27,400 \text { cal. BP }\end{array}$ & Pounded and pecked bird track (K21) & $\begin{array}{l}\text { Dorn and Nobbs, } \\
\text { 1992; Dorn et } \\
\text { al., 1992; Nobbs } \\
\text { and Dorn } 1993\end{array}$ \\
\hline Karolta $1, \mathrm{SA}^{*}$ & $\begin{array}{l}\text { AMS: } \geq 21,195 \pm 220 \mathrm{BP}(\mathrm{AA}- \\
6905) \\
2 \sigma: 24,974-25,927 \mathrm{cal} . \mathrm{BP}\end{array}$ & Pounded and pecked track (K32) & $\begin{array}{l}\text { Nobbs and Dorn, } \\
1993\end{array}$ \\
\hline Karolta $1, \mathrm{SA}^{*}$ & $\begin{array}{l}\text { AMS: } \geq 20,105 \pm 185 \mathrm{BP}(\mathrm{AA}- \\
6548) \\
2 \sigma: 23,636-24,581 \mathrm{cal} . \mathrm{BP}\end{array}$ & Pounded and pecked foot print (K30) & $\begin{array}{l}\text { Nobbs and Dorn, } \\
1993\end{array}$ \\
\hline
\end{tabular}




\begin{tabular}{|c|c|c|c|}
\hline $\begin{array}{l}\text { Wharton Hill, } \\
\text { SA* }\end{array}$ & $\begin{array}{l}\text { AMS: } \geq 18,485 \pm 165 \text { BP (AA- } \\
6918) \\
2 \sigma: 21,879-22,643 \text { cal. BP }\end{array}$ & Pounded and pecked footprint (WH2) & $\begin{array}{l}\text { Nobbs and Dorn, } \\
1993\end{array}$ \\
\hline $\begin{array}{l}\text { Laurie Creek, } \\
\text { NT* }^{*}\end{array}$ & $\begin{array}{l}\text { AMS: } 20,320+3100 /-2300 \\
\text { (RIDDL-1270) } \\
2 \sigma: 18,837-37,679 \text { cal. BP }\end{array}$ & Painted hand stencil & $\begin{array}{l}\text { Gillespie, 1997; } \\
\text { Loy et al., } 1990\end{array}$ \\
\hline $\begin{array}{l}\text { Gum Tree } \\
\text { Valley, WA }\end{array}$ & $\begin{array}{l}\text { LSC: } 18,510 \pm 260 \text { BP (LY-3609) } \\
2 \sigma: 21,530-22,223 \text { cal. BP }\end{array}$ & $\begin{array}{l}\text { Syrinx aruanus shell found in a fissure among } \\
\text { deeply patinated engravings of ghost-like } \\
\text { figures, kangaroos, geometrics. Note that the } \\
\text { association of the dated shell with the } \\
\text { engravings is unclear. }\end{array}$ & $\begin{array}{l}\text { Lorblanchet, } \\
\text { 1992; Rosenfeld, } \\
\text { 1993 }\end{array}$ \\
\hline $\begin{array}{l}\text { Walkunder Arch } \\
\text { Cave, QLD }\end{array}$ & $\begin{array}{l}\text { AMS: } 16,100 \pm 130 \text { BP (OZA- } \\
395) \\
2 \sigma: 18,746-20,114 \text { cal. BP }\end{array}$ & Red pigment on rock wall & $\begin{array}{l}\text { Campbell et al., } \\
1996\end{array}$ \\
\hline $\begin{array}{l}\text { Wharton Hill, } \\
\text { SA* }\end{array}$ & $\begin{array}{l}\text { AMS: } \geq 14,910 \pm 180 \text { BP (NZA- } \\
1367) \\
2 \sigma: 17,659-18,526 \text { cal. BP }\end{array}$ & $\begin{array}{l}\text { Pounded and pecked possible Drominorthid } \\
\text { track (WH1) }\end{array}$ & $\begin{array}{l}\text { Dorn and Nobbs, } \\
\text { 1992; Nobbs and } \\
\text { Dorn, } 1993\end{array}$ \\
\hline $\begin{array}{l}\text { Yunta Springs, } \\
\text { SA* }\end{array}$ & $\begin{array}{l}\text { AMS: } \geq 13,950 \pm 110 \text { BP (AA- } \\
6914) \\
2 \sigma: 16,447-17,225 \text { cal. BP }\end{array}$ & $\begin{array}{l}\text { Pounded and pecked complex curvilinear motif } \\
\text { (YS1) }\end{array}$ & $\begin{array}{l}\text { Nobbs and Dorn, } \\
1993\end{array}$ \\
\hline Puritjarra, NT & $\begin{array}{l}\text { LSC: } \leq 13,570 \pm 100 \text { BP (ANU- } \\
7460) \\
2 \sigma: 16,131-16,455 \text { cal. BP }\end{array}$ & $\begin{array}{l}\text { Pecked and weathered circles and incised lines } \\
\text { on boulders embedded in shelter floor }\end{array}$ & $\begin{array}{l}\text { Rosenfeld, 1999; } \\
\text { Rosenfeld and } \\
\text { Smith, 2002; } \\
\text { Smith, 2006 }\end{array}$ \\
\hline Karolta $1, \mathrm{SA}^{*}$ & $\begin{array}{l}\text { AMS: } \geq 12,970 \pm 150 \text { BP (NZA- } \\
1414) \\
2 \sigma: 15,031-15,966 \text { cal. BP }\end{array}$ & Pounded and pecked abstract motif (K24) & $\begin{array}{l}\text { Dorn and Nobbs, } \\
\text { 1992; Dorn et } \\
\text { al., 1992; Nobbs } \\
\text { and Dorn, } 1993\end{array}$ \\
\hline Karolta $1, \mathrm{SA}^{*}$ & $\begin{array}{l}\text { AMS: } \geq 12,650 \pm 150 \text { BP (NZA- } \\
1369) \\
2 \sigma: 14,190-15,380 \text { cal. BP }\end{array}$ & Pounded and pecked curved line (K15) & $\begin{array}{l}\text { Dorn and Nobbs, } \\
\text { 1992; Nobbs and } \\
\text { Dorn, } 1993\end{array}$ \\
\hline $\begin{array}{l}\text { Keyhole Cavern, } \\
\text { TAS }\end{array}$ & $>\mathrm{c} .13,000 \mathrm{BP}$ & 3 red hand stencils, ochre smears & $\begin{array}{l}\text { McGowan et al., } \\
\text { 1993; Morwood } \\
2002\end{array}$ \\
\hline $\begin{array}{l}\text { Ballawinne, } \\
\text { TAS }\end{array}$ & $>\mathrm{c} .13,000 \mathrm{BP}$ & 23 red hand stencils, ochre smears and patches & $\begin{array}{l}\text { Harris et al., } \\
\text { 1988; McGowan } \\
\text { et al., 1993; } \\
\text { Morwood } 2002\end{array}$ \\
\hline $\begin{array}{l}\text { Magnificent } \\
\text { Gallery, QLD }\end{array}$ & $\begin{array}{l}\text { 14C: } 10,250 \pm 90 \text { (SUA-2876) } \\
2 \sigma: 11,621-12,400 \text { cal. BP }\end{array}$ & $\begin{array}{l}\text { Early figurative paintings - human figures, } \\
\text { crosses, hand stencils }\end{array}$ & Morwood, 1989 \\
\hline $\begin{array}{l}\text { Eight Mile } \\
\text { Creek, SA }\end{array}$ & $\begin{array}{l}\text { AMS: } 10,250 \pm 170 \text { (Beta- } 13803) \\
2 \sigma: 11,415-12,382 \text { cal. BP }\end{array}$ & Pecking motifs & $\begin{array}{l}\text { Clegg, 1987; } \\
\text { Dragovich, } \\
\text { 1986; Rosenfeld, } \\
1993\end{array}$ \\
\hline
\end{tabular}




\begin{tabular}{|l|l|l|l|}
\hline & $\begin{array}{l}\text { AMS: } 9240 \pm 820 \text { BP (RIDDL- } \\
1269) \\
2 \sigma: 8552-12,753 \text { cal. BP }\end{array}$ & Wargata Mina, \\
TAS* & to & 23 hand stencils, ochre smears and patches & $\begin{array}{l}\text { Jones et al., } \\
\text { 1988; Loy et al., } \\
\text { 1990; McGowan } \\
\text { et al., 1993 }\end{array}$ \\
& $\begin{array}{l}\text { AMS: } 10,730 \pm 810 \text { BP (RIDDL- } \\
1268) \\
2 \sigma: 10,285-14,859 \text { cal. BP }\end{array}$ & & \\
\hline Karolta 1, SA* & $\begin{array}{l}\text { AMS: } \geq 9,980 \pm 85 \text { BP (AA-6910) } \\
2 \sigma: 11,206-11,749 \text { cal. BP }\end{array}$ & Pounded and pecked foot print (K29) & $\begin{array}{l}\text { Nobbs and Dorn, } \\
1993\end{array}$ \\
\hline Karolta 1, SA* & $\begin{array}{l}\text { AMS: } \geq 9,125 \pm 100 \text { BP (AA- } \\
6916) \\
2 \sigma: 9,914-10,517 \text { cal. BP }\end{array}$ & Pounded and pecked hand print (K28a) & $\begin{array}{l}\text { Nobbs and Dorn, } \\
1993\end{array}$ \\
\hline
\end{tabular}

The chronology of early pecked engravings is very uncertain, with initial dating attempts using cation-ration and radiocarbon approaches subject to heavy critique (see Beck et al., 1998; Watchman, 1992). Until further research is undertaken, we recommend disregarding the cation-ratio and radiocarbon dates presented Nobbs and Dorn (1993; see also Dorn and Nobbs, 1992; Dorn et al. 1992). Some Panaramittee images, originally believed to represent the earliest art of Australia, are now thought to be Holocene in age (Smith, 2013; Smith et al., 2009). Indeed, 'Panaramittee' may be more of an archaeological construct rather than a cultural reality (Rosenfeld, 1991). Other early art styles are argued to include pecked 'archaic faces' (Mulvaney, 2013) along with painted panels representing both individuals and groups dancing or fighting while wearing complex headdresses and holding items such as barbed spears and baskets, along with large naturalist depictions of animals (Flood, 1997; Taçon and Chippindale, 1994; Walshe, 1994; Mulvaney, 2013).

The oldest directly dated rock art is at Gnatalia Creek where a large red and black curvilinear non-figurative drawing was dated to 33,066-34,675 cal. BP (AA-5851), although another sample from the same motif returned an age of 6735-7156 cal. BP, indicating the possible presence of contaminants (McDonald et al., 1990; Rosenfeld, 1993). Claims for directly dated blood proteins in art at Laurie Creek and Wargata Mina reported by Loy et al. (1990) have been disputed (Gillespie, 1997; Nelson, 1993).

The fragmentary nature of the earliest rock art evidence in Sahul makes identifying the nature of the earliest images challenging, although we were able to record 39 instances. Most of these examples are associated with direct dates, though many are contested and must be treated with caution (Table 3). 


\subsection{Portable Art}

The two 'engraved' limestone plaques dated to $23,470-25,710$ cal. BP and 13,576-14,067 cal. BP respectively recovered from Devil's Lair (Dortch, 1976), remain the only examples of incised 'notational' pieces in stone (Table 4). One displays a trapezoidal shape while is not clear in its design. These plaques remain ambiguous in terms of their anthropogenic origins, and may yet prove to have a natural origin (see Bednarik, 1998 for discussion). Two other instances of what would traditionally be termed 'notational pieces' include a macropod femur with incised grooves dated to 17,905-25,725 cal. BP from Cave Bay Cave, King Island (Bowdler, 1984) and a Diprotodon optatum incisor with 28 incised grooves found at Spring Creek, Victoria dated to 32,602 cal. BP (Vanderwal and Fullagar, 1989). This last item, the Diprotodon incisor, has recently been re-examined and found to be the product of natural processes (Langley and Rybachuk, in prep.).

Table 4: Current evidence for the Pleistocene examples of portable art in Sahul.

\begin{tabular}{|c|c|c|c|}
\hline \multicolumn{4}{|l|}{ Portable Art } \\
\hline Site & Age & Evidence & Source \\
\hline $\begin{array}{l}\text { Cave Bay Cave, } \\
\text { TAS }\end{array}$ & $\begin{array}{l}\text { LSC: } 15,400 \pm 330 \text { BP (ANU- } \\
\text { 1613) } \\
2 \sigma: 17,902-19,416 \text { cal. BP } \\
\text { to } \\
\text { LSC: } 20,850 \pm 290 \text { BP (ANU- } \\
1612 \text { ) } \\
2 \sigma: 24,345-25,725 \text { cal. BP }\end{array}$ & $\begin{array}{l}\text { Macropod femur with groups of grooves and } \\
\text { scratches }\end{array}$ & Bowdler, 1984 \\
\hline $\begin{array}{l}\text { Devil's Lair, } \\
\text { WA }\end{array}$ & $\begin{array}{l}\text { LSC: } 20,400 \pm 1000 \text { BP (SUA- } \\
\text { 32) } \\
\text { 2ఠ: } 23,470-25,710 \text { cal. BP }\end{array}$ & $\begin{array}{l}\text { Limestone plaque (B3652) engraved with } \\
\text { incisions and geometric shapes }\end{array}$ & Dortch, 1976 \\
\hline $\begin{array}{l}\text { Devil's Lair, } \\
\text { WA }\end{array}$ & $\begin{array}{l}\text { LSC: } 11,960 \pm 140 \text { BP (SUA- } \\
\text { 102) } \\
2 \sigma: 13,576-13,931 \text { cal. BP } \\
\text { to } \\
\text { LSC: } 12,050 \pm 140 \text { BP (SUA- } \\
\text { 103) } \\
2 \sigma: 13,718-14,067 \text { cal. BP }\end{array}$ & $\begin{array}{l}\text { Limestone plaque (B3651) engraved with a } \\
\text { trapezoidal shape and incisions }\end{array}$ & Dortch, 1976 \\
\hline
\end{tabular}




\subsection{Burial}

Sixteen instances of Pleistocene burials (including burials of multiple individuals) are documented (Table 5). The majority of these occur in the Willandra Lakes system, New South Wales and include the oldest known cremation in the world (LM1 dated to 42.5 $\pm 2.4-46.6 \pm 2.3 \mathrm{ka}$ ) (Bowler et al., 2003), and LM3 a ritual extended burial (inferred from the use of red pigment over the body and clasped hands) dating to either c.40,000 years or 62,000 years based on U-series and ESR methods (Bowler et al., 2003; Oyston 1996; Thorne et al., 1999; Gillespie and Roberts 2000). Apart from these examples, alongside another from Lake Urana dated to c.25-30 ka, all remaining burials date to the final 5,000 years of the Pleistocene. Three of these later instances appear to be cemeteries with multiple burials (Coobool Creek: c.14,300 1000 BP, Kow Swamp: c.26,0000-14,000, and Tuckers Creek: c.14,000-15,000 cal. BP) (Bowler, 1983; Clark and Hope, 1985; Meehan, 1984; Pardoe, 1993b). Burials display a range of practices including extended (LM3), cremated (LM1), and kneeling (Lake Tandou) positions, and a number include grave goods such as mussel shell, ochre, lithics (Kow Swamp, Lake Mungo, Tuckers Creek), and burial cairns (Cliff Cave).

Table 5: Current evidence for Pleistocene burials in Sahul.

\begin{tabular}{|c|c|c|c|}
\hline \multicolumn{4}{|l|}{ Burials } \\
\hline Site & Age & Evidence & Source \\
\hline $\begin{array}{l}\text { Lake Mungo, } \\
\text { NSW }\end{array}$ & $\begin{array}{l}\text { OSL: } 42.5 \pm 2.4 \mathrm{ka}(\mathrm{M} 1 \mathrm{R} \\
0.37 \mathrm{~m} / \mathrm{a}) \\
\text { to } \\
\text { OSL: } 46.6 \pm 2.3 \mathrm{ka}(\mathrm{M} 1 \mathrm{R} \\
0.0 \mathrm{~m})\end{array}$ & LM1 - cremation with associated ochre & $\begin{array}{l}\text { Bowler et al., } \\
\text { 1970; Bowler et } \\
\text { al., } 2003\end{array}$ \\
\hline $\begin{array}{l}\text { Lake Mungo, } \\
\text { NSW* }^{*}\end{array}$ & $\begin{array}{l}\text { OSL: } 38.2 \pm 1.3 \mathrm{ka}(\mathrm{M} 3 \mathrm{~T} 61.2 \\
\mathrm{m}) \\
\text { to } \\
\text { OSL: } 42.2 \pm 2.5 \mathrm{ka}(\mathrm{M} 3 \mathrm{~T} 58.0 \\
\mathrm{m})\end{array}$ & LM3 - extended burial covered with ochre & $\begin{array}{l}\text { Bowler et al., } \\
\text { 2003; Thorne et } \\
\text { al., } 1999\end{array}$ \\
\hline $\begin{array}{l}\text { Lake Urana, } \\
\text { NSW }\end{array}$ & $\begin{array}{l}\text { OSL: } 25.6 \pm 7.3 \mathrm{ka}(\mathrm{W} 829) \\
\text { to } \\
\text { OSL: } 32.4 \pm 8 \mathrm{ka}(\mathrm{W} 830)\end{array}$ & Possible burial of an adult female & Page et al., 1994 \\
\hline $\begin{array}{l}\text { Liang Lemdubu, } \\
\text { NG }\end{array}$ & ESR: $18,800 \pm 2,300$ & $\begin{array}{l}\text { Burial of an adult female partially sealed by a } \\
\text { flat stone }\end{array}$ & $\begin{array}{l}\text { Bulbeck, 2005; } \\
\text { O’Connor et al., } \\
2005 b\end{array}$ \\
\hline $\begin{array}{l}\text { Lake Tandou, } \\
\text { NSW }\end{array}$ & $\begin{array}{l}\text { LSC: } 15,210 \pm 160 \mathrm{BP}(\mathrm{SUA}- \\
1805) \\
2 \sigma: 18,028-18,761 \mathrm{cal} . \mathrm{BP}\end{array}$ & Kneeling burial of a male & $\begin{array}{l}\text { Freedman and } \\
\text { Lofgren, } 1983\end{array}$ \\
\hline Cliff Cave, TAS & $\begin{array}{l}\text { LSC: } 14,270 \pm 640 \text { BP (ANU- } \\
7039) \\
2 \sigma: 15,665-18,931 \text { cal. BP }\end{array}$ & $\begin{array}{l}\text { Burial cairn containing skeletal remains, } \\
\text { including red ochre on the cranium and femur }\end{array}$ & $\begin{array}{l}\text { Sim and Thorne, } \\
1990\end{array}$ \\
\hline
\end{tabular}




\begin{tabular}{|c|c|c|c|}
\hline $\begin{array}{l}\text { Lake Benanee, } \\
\text { NSW }\end{array}$ & $\begin{array}{l}\text { LSC: } 13,200 \pm 130 \text { BP (ANU- } \\
4315) \\
2 \sigma: 15,336-16,166 \text { cal. BP }\end{array}$ & 2 burials under shell midden & $\begin{array}{l}\text { Clark and Hope, } \\
1985\end{array}$ \\
\hline $\begin{array}{l}\text { Tuckers Creek, } \\
\text { NSW }\end{array}$ & $\begin{array}{l}\text { LSC: } 12,600 \pm 130 \text { BP (ANU- } \\
4317) \\
2 \sigma: 14,210-15,246 \text { cal. BP }\end{array}$ & $>17$ burials found in crouched positions & $\begin{array}{l}\text { Clark and Hope, } \\
1985\end{array}$ \\
\hline $\begin{array}{l}\text { Lake Tandou, } \\
\text { NSW }\end{array}$ & $\begin{array}{l}\text { LSC: } 12,530+1630 /-1350 \mathrm{BP} \\
\text { (ANU-705) } \\
2 \sigma: 11,274-19,615 \text { cal. BP }\end{array}$ & Cremation & Allen, 1972 \\
\hline $\begin{array}{l}\text { Tandou Creek, } \\
\text { NSW }\end{array}$ & $\begin{array}{l}\text { LSC: } 12,350 \pm 170 \mathrm{BP}(\mathrm{ANU}- \\
702) \\
2 \sigma: 13,812-15,048 \mathrm{cal} . \mathrm{BP}\end{array}$ & Single burial & Allen, 1972 \\
\hline Talgai, QLD & $\begin{array}{l}\text { LSC: } 12400 \pm 300 \mathrm{BP} \\
(\mathrm{R} 2526 / 29) \\
2 \sigma: 13,588-15,445 \mathrm{cal} . \mathrm{BP}\end{array}$ & 15-16-year-old boy & $\begin{array}{l}\text { Brown, 1987; } \\
\text { Pardoe, 1993a }\end{array}$ \\
\hline Nacurrie, NSW & $\begin{array}{l}\text { LSC: } 11,440 \pm 160 \mathrm{BP} \text { (not } \\
\text { available) } \\
2 \sigma: 12,926-13,569 \mathrm{cal} . \mathrm{BP}\end{array}$ & Burial of a male & Pardoe, 1993a \\
\hline $\begin{array}{l}\text { Kow Swamp, } \\
\text { VIC }\end{array}$ & $\begin{array}{l}\text { OSL: } 14.4 \pm 0.8 \mathrm{ka}(\mathrm{RP} 1) \\
\text { to } \\
\text { OSL: } 26 \pm 2 \mathrm{ka}(\mathrm{RP} 4)\end{array}$ & $\begin{array}{l}\text { Burial ground with }>40 \text { individuals, some } \\
\text { buried with grave goods including a girl or } \\
\text { young woman wearing a kangaroo incisor } \\
\text { headband }\end{array}$ & $\begin{array}{l}\text { Pardoe, 1993a; } \\
\text { Stone and } \\
\text { Cupper, } 2003\end{array}$ \\
\hline $\begin{array}{l}\text { Keilor, Dry } \\
\text { Creek, VIC }\end{array}$ & $\begin{array}{l}\text { LSC: } 12,000 \pm 100 \mathrm{BP}(\mathrm{NZ}- \\
1327) \\
2 \sigma: 13,561-14,068 \mathrm{cal} . \mathrm{BP}\end{array}$ & Adult male & $\begin{array}{l}\text { Brown, 1987; } \\
\text { Pardoe, 1993a }\end{array}$ \\
\hline $\begin{array}{l}\text { Snaggy Bend, } \\
\text { NSW }\end{array}$ & $\begin{array}{l}\text { LSC: } 10,000 \pm 120 \text { BP (ANU- } \\
3794) \\
2 \sigma: 11,187-11,947 \text { cal. BP }\end{array}$ & Burial 79 & $\begin{array}{l}\text { Clark and Hope, } \\
1985\end{array}$ \\
\hline $\begin{array}{l}\text { Coobool Creek, } \\
\text { NSW }\end{array}$ & $\begin{array}{l}\mathrm{U}: 14,300 \pm 1000 \text { BP (LLO- } \\
416)\end{array}$ & Burial ground with 126 individuals & $\begin{array}{l}\text { Brown, 1987; } \\
\text { Pardoe, 1993a }\end{array}$ \\
\hline
\end{tabular}




\section{Current Evidence: Sunda and Wallacea}

Tabling the current evidence for pre-Neolithic symbolic cultural behaviours in the Sunda and Wallacea area is hindered by a lack of rigorous dating, with the exception of excavation programs undertaken in the past 10-15 years (e.g., O'Connor, 2007; O’Connor et al., 2010; van den Bergh et al., 2016). In many cases, items which we would consider to be of 'symbolic' value are simply noted as being recovered from terminal Pleistocene-early Holocene contexts such as the Hoabinhian. Defining the Hoabinhian is difficult as its chronological and geographical distribution is yet to be clearly defined. Most simply, it can be described as a late Pleistocene-early Holocene industry of Southeast Asia which is characterised by unifacial flaked tools made primarily on water rounded pebbles, core tools ('sumatraliths'), and grinding stones (Chitkament et al. 2016; Ji et al. 2016; Marwick, 2018). In other cases, clear description of pieces lack the level of detail required to situate them among their contemporaries. These trends are highlighted in the following overview.

\subsection{Colourants}

Recent excavations in Sunda and Wallacea have resulted in a significant increase in the number of 'symbolic' items reported in the archaeological literature (see O'Connor and Langley, 2018). In terms of colourants, evidence can again be grouped into finds of utilised pigments, pigment-stained artefacts, and ochre stained burials. The earliest instances of colourants in this region are found on two examples of worked and ochre stained Nautilus pompilius fragments recovered from contexts dated to between $38,432-$ 42,117 cal. BP at Jerimalai in Timor-Leste (Langley et al., 2016b). Similar finds of ochre stained Nautilus fragments and fully formed beads are found in younger contexts (>15,900, 9,500, and 5,000 cal. BP) at Jerimalai. Also found were a number of Oliva spp. shell beads present from around 16,000 cal. BP until the recent past. These bare traces of red colourant consistent with incidental transfer of pigment to the beads's surfaces (Table 6; Langley and O'Connor, 2016). This type of marine shell bead was also found in significant numbers throughout the deposits of the nearby sites of Matju Kuru 1 and Matju Kuru 2, though only small numbers were recovered from the Pleistocene levels (Table 6).

The oldest known examples of utilised mineral pigments in Sunda and Wallacea come from the site of Jerimalai, with examples found from the earliest levels of the site, dating to 38,432-42,117 cal. BP, through the recent past. While dark red shades dominate the assemblage, several black and yellow pieces are also present (Langley and O'Connor, 2019). Dating to between c.21,000 and 26,000 cal. BP, is a collection of red ochres recovered from the Sulawesi site of Leang Bulu Bettue. Here, red and mulberry coloured ochre fragments were found to have use wear consistent with scraping to produce powder, with some also showing traces of having been rubbed on animal hide or human skin. Ochre stained lithics were also identified, as was a possible pigment blow pipe made on the long bone of a bear cuscus (Brumm et al., 2017). Significantly, the earliest indications of pigment use at Leang Bulu Bettue - two ochre-stained chert tools from Layer $4 \mathrm{f}$ - are consistent in age with the oldest known parietal art motif in Maros-Pangkep (Leang Timpuseng, c.39,900 cal. BP), while the ochre nodules from Layer 4a (dated to c.21,000-26,000 cal. BP) fit well with the latest dated hand stencil in this Maros-Pankep karsts (Aubert et al., 2014; Brumm et al., 2017). Similarly aged (associated with an age of 25,896-26,520 cal. BP) examples of utilised ochre have also been found at nearby Leang Burung 2 (Glover, 1981), while a chert flake with ochre residues is known from Leang Sakapao 1 (dated to $\sim 28,605-36,405$ cal. BP) (see Bulbeck et al., 2004 for a discussion of issues with the chronology arising from freshwater shell carbon reservoir issues). 
In the Niah Caves of Borneo, a fragment of hard-shelled turtle plastron displays a pigmented section with a straight and clearly defined perimeter suggesting intentional execution. This artefact comes from layers dated to 39,880-41,601 cal. BP (Reynolds et al., 2013). At the cave site of Xom Trai, located in the Lac Son District of Hoa Bihn Province of Vietnam, ochre and kaolinite stones exhibiting evidence for grinding or scraping date to between 22,000 and 19,000 cal. BP (Nguyen, 2000 cited in Rabett and Piper, 2012). Also in Vietnam, thousands of red ochre fragments are found in the Hoabinhian levels of Du Sang Save, located on the west margin of the Khang Valley (Nguyen, 2015).

Table 6: Current evidence for the Pleistocene examples of ochre use in Sunda and Wallacea.

\begin{tabular}{|c|c|c|c|}
\hline \multicolumn{4}{|l|}{ Utilised Ochre } \\
\hline Site & Age & Evidence & Source \\
\hline $\begin{array}{l}\text { Jerimalai, } \\
\text { Timor-Leste }\end{array}$ & $\begin{array}{l}\text { AMS: } 35,387 \pm 534 \text { BP (Wk- } \\
\text { 19232) } \\
2 \sigma: 38,432-40,749 \text { cal. BP } \\
\text { to } \\
\text { AMS: } 37,267 \pm 453 \text { BP (Wk- } \\
17833) \\
2 \sigma: 40,486-42,117 \text { cal. BP }\end{array}$ & 3 pieces of utilised ochre & $\begin{array}{l}\text { Langley and } \\
\text { O'Connor, } 2019\end{array}$ \\
\hline $\begin{array}{l}\text { Matja Kuru 2, } \\
\text { Timor-Leste }\end{array}$ & $\begin{array}{l}\text { AMS: } 31,060 \pm 130 \text { BP (NZA- } \\
\text { 16177) } \\
2 \sigma: 34,190-34,849 \text { cal. BP } \\
\text { to } \\
\text { AMS: } 32,200 \pm 300 \text { BP (OZF- } \\
785) \\
2 \sigma: 34,960-36,260 \text { cal. BP }\end{array}$ & 6 pieces of utilised ochre & $\begin{array}{l}\text { Langley and } \\
\text { O'Connor, } 2019\end{array}$ \\
\hline $\begin{array}{l}\text { Lene Hara, } \\
\text { Timor-Leste }\end{array}$ & $\begin{array}{l}\text { AMS: } \geq 30110 \pm 320 \text { BP (ANU- } \\
11398) \\
2 \sigma: 33,115-34,415 \text { cal. BP } \\
\text { to } \\
\text { AMS: } 30,970 \pm 460 \text { BP (ANU- } \\
11420) \\
33,751-35,415 \text { cal. BP }\end{array}$ & 3 pieces of utilised ochre & $\begin{array}{l}\text { Langley and } \\
\text { O'Connor, } 2019\end{array}$ \\
\hline $\begin{array}{l}\text { Leang Bulu } \\
\text { Bettue, } \\
\text { Sulawesi }\end{array}$ & $\begin{array}{l}\text { AMS: } 18,126 \pm 51 \mathrm{BP}(\mathrm{Wk}- \\
37742) \\
2 \sigma: 21,792-22,196 \mathrm{cal} . \mathrm{BP} \\
\text { to } \\
\mathrm{U}: 25.9 \pm 0.7 \mathrm{ka}(605-1900)\end{array}$ & $\begin{array}{l}4 \text { nodules of utilised ochre exhibiting } \\
\text { traces of grinding and scraping }\end{array}$ & Brumm et al., 2017 \\
\hline
\end{tabular}




\begin{tabular}{|c|c|c|c|}
\hline $\begin{array}{l}\text { Leang Burung } \\
2 \text {, Sulawesi }\end{array}$ & $\begin{array}{l}\text { LSC: } 23,300 \pm 140 \mathrm{BP}(\mathrm{GrN}- \\
8293) \\
2 \sigma: 25,896-26,520 \mathrm{cal} . \mathrm{BP}\end{array}$ & $\begin{array}{l}\text { Two pieces of abraded ochreous } \\
\text { ironstone, one of which has cross-cutting } \\
\text { parallel grooves. Note that a hard water } \\
\text { correction of } 1350 \text { years was subtracted } \\
\text { from the conventional radiocarbon age } \\
\text { before calibration with IntCal13. }\end{array}$ & Glover, 1981 \\
\hline $\begin{array}{l}\text { Jerimalai, } \\
\text { Timor-Leste }\end{array}$ & $\begin{array}{l}\text { AMS: }>19,952 \pm 235 \mathrm{BP}(\mathrm{Wk}- \\
17830) \\
2 \sigma: 22,870-24,058 \mathrm{cal} . \mathrm{BP}\end{array}$ & 1 piece of utilised ochre & $\begin{array}{l}\text { Langley and } \\
\text { O’Connor, } 2019\end{array}$ \\
\hline $\begin{array}{l}\text { Lene Hara, } \\
\text { Timor-Leste }\end{array}$ & $\begin{array}{l}\text { AMS: } 18,740 \pm 400 \text { BP (ANU- } \\
12138) \\
2 \sigma: 21,052-23,043 \text { cal. BP }\end{array}$ & 1 piece of utilised ochre & $\begin{array}{l}\text { Langley and } \\
\text { O’Connor, } 2019\end{array}$ \\
\hline $\begin{array}{l}\text { Xom Trai, } \\
\text { Vietnam }\end{array}$ & $\begin{array}{l}16,130 \pm 90 \mathrm{BP}(\mathrm{B} \ln -3042) \\
\text { to } \\
18,420 \pm 150(\mathrm{~B} \ln -3472)\end{array}$ & $\begin{array}{l}\text { Ochre and kaolinite nodules exhibiting } \\
\text { traces of grinding, scraping. }\end{array}$ & $\begin{array}{l}\text { Ngyuen, 2015; Rabett } \\
\text { and Piper, } 2012\end{array}$ \\
\hline $\begin{array}{l}\text { Jerimalai, } \\
\text { Timor-Leste }\end{array}$ & $\begin{array}{l}\text { AMS: } 13,901 \pm 45 \text { BP }(\mathrm{Wk}- \\
30502) \\
2 \sigma: 15,875-16,425 \text { cal. BP }\end{array}$ & 1 piece of utilised ochre & $\begin{array}{l}\text { Langley and } \\
\text { O’Connor, } 2019\end{array}$ \\
\hline $\begin{array}{l}\text { Matja Kuru 2, } \\
\text { Timor-Leste }\end{array}$ & $\begin{array}{l}\text { AMS: } 9650 \pm 55 \text { BP (NZA-16137) } \\
2 \sigma: 10,226-10,656 \mathrm{cal} . \mathrm{BP} \\
\text { to } \\
\text { AMS: } 11,173 \pm 55 \mathrm{BP}(\mathrm{NZA}- \\
\text { 16138) } \\
2 \sigma: 12,457-12,794 \mathrm{cal} . \mathrm{BP}\end{array}$ & 18 pieces of utilised ochre & $\begin{array}{l}\text { Langley and } \\
\text { O'Connor, } 2019\end{array}$ \\
\hline $\begin{array}{l}\text { Lene Hara, } \\
\text { Timor-Leste }\end{array}$ & $\begin{array}{l}\text { AMS: } 9741 \pm 60 \text { BP (NZA-17000) } \\
2 \sigma: 10,275-10,800 \text { cal. BP } \\
\text { to } \\
\text { AMS: } 10,050 \pm 80 \text { BP (ANU- } \\
12040 \\
2 \sigma: 10,680-11,185 \mathrm{cal} . \mathrm{BP}\end{array}$ & 2 pieces of utilised ochre & $\begin{array}{l}\text { Langley and } \\
\text { O'Connor, } 2019\end{array}$ \\
\hline $\begin{array}{l}\text { Du Sang, } \\
\text { Vietnam }\end{array}$ & Hoabinhian & Red ochre fragments & Ngyuen, 2015 \\
\hline \multicolumn{4}{|c|}{ Ochre-Stained Artefacts } \\
\hline $\begin{array}{l}\text { Jerimalai, } \\
\text { Timor-Leste }\end{array}$ & $\begin{array}{l}\text { AMS: } 35,387 \pm 534 \text { BP (Wk- } \\
\text { 19232) } \\
2 \sigma: 38,432-40,749 \text { cal. BP } \\
\text { to } \\
\text { AMS: } 37,267 \pm 453 \mathrm{BP}(\mathrm{Wk}- \\
17833) \\
2 \sigma: 40,486-42,117 \mathrm{cal} . \mathrm{BP}\end{array}$ & $\begin{array}{l}\text { Two worked and ochre stained pieces of } \\
\text { Nautilus pompilius - likely ornamental. }\end{array}$ & Langley et al., 2016b \\
\hline
\end{tabular}




\begin{tabular}{|c|c|c|c|}
\hline $\begin{array}{l}\text { Niah Caves, } \\
\text { Sarawak }\end{array}$ & $\begin{array}{l}\text { AMS: } 35,890 \pm 250 \mathrm{BP}(\mathrm{OxA}- \\
15163) \\
2 \sigma: 39,931-41,162 \mathrm{cal} . \mathrm{BP} \\
\text { to } \\
\text { AMS: } 36,470 \pm 250 \mathrm{BP}(\mathrm{OxA}- \\
15164) \\
2 \sigma: 40,526-41,635 \mathrm{cal} . \mathrm{BP}\end{array}$ & $\begin{array}{l}\text { Fragment of hard-shelled turtle plastron } \\
\text { displaying a pigmented section with a } \\
\text { straight and clearly defined perimeter }\end{array}$ & Reynolds et al., 2013 \\
\hline $\begin{array}{l}\text { Niah Caves, } \\
\text { Sarawak }\end{array}$ & $\begin{array}{l}\text { AMS: }>36,470 \pm 250 \mathrm{BP}(\mathrm{OxA}- \\
15164) \\
2 \sigma: 40,526-41,635 \mathrm{cal} . \mathrm{BP}\end{array}$ & $\begin{array}{l}3 \text { human cranial fragments with red } \\
\text { pigment staining on inner surface, } \\
\text { probably a tree resin }\end{array}$ & $\begin{array}{l}\text { Pyatt et al. 2010; } \\
\text { Reynolds et al., } 2013\end{array}$ \\
\hline $\begin{array}{l}\text { Leang Sakapao } \\
1, \text { Sulawesi* }\end{array}$ & $\begin{array}{l}\text { LSC: } 25,120 \pm 260 \mathrm{BP}(\mathrm{Wk}- \\
4261) \\
2 \sigma: 28,605-29,830 \mathrm{cal} . \mathrm{BP} \\
\text { to } \\
\text { LSC: } 31,280 \pm 570 \mathrm{BP}(\mathrm{Wk}-3821) \\
2 \sigma: 34,157-36,405 \mathrm{cal} . \mathrm{BP}\end{array}$ & $\begin{array}{l}\text { Chert flake (LSK-3-208) covered with } \\
\text { orange ochre residues }\end{array}$ & Bulbeck et al., 2004 \\
\hline $\begin{array}{l}\text { Leang Bulu } \\
\text { Bettue, } \\
\text { Sulawesi }\end{array}$ & $\begin{array}{l}\text { AMS: } 18,126 \pm 51 \mathrm{BP}(\mathrm{Wk}- \\
37742) \\
2 \sigma: 21,792-22,196 \mathrm{cal} . \mathrm{BP} \\
\text { to } \\
\mathrm{U:} 39.8 \pm 0.2 \mathrm{ka}(3612)\end{array}$ & 30 chert artefacts with ochre residues & Brumm et al., 2017 \\
\hline $\begin{array}{l}\text { Leang Bulu } \\
\text { Bettue, } \\
\text { Sulawesi }\end{array}$ & $\begin{array}{l}\mathrm{U}: 24.6 \pm 0.2 \mathrm{ka}(605-0274 \\
\text { to } \\
\mathrm{U}: 25.9 \pm 0.7 \mathrm{ka}(605-1900)\end{array}$ & $\begin{array}{l}\text { Perforated Ailurops ursinus phalange } \\
\text { long bone with red and black pigments } \\
\text { inside and on one end (possible blow- } \\
\text { pipe). }\end{array}$ & Brumm et al., 2017 \\
\hline $\begin{array}{l}\text { Jerimalai, } \\
\text { Timor-Leste }\end{array}$ & $\begin{array}{l}\text { AMS: c. } 10,110 \pm 79 \text { BP }(\mathrm{Wk}- \\
\text { 18156) } \\
2 \sigma: 10,726-11,229 \mathrm{cal} \mathrm{BP} \\
\text { to } \\
\text { AMS: c. } 13,658 \pm 91 \mathrm{BP}(\mathrm{Wk}- \\
19227) \\
2 \sigma: 15,413-16,175 \mathrm{cal} . \mathrm{BP}\end{array}$ & $\begin{array}{l}8 \text { Oliva sp. shell beads with red ochre } \\
\text { staining }\end{array}$ & $\begin{array}{l}\text { Langley and } \\
\text { O'Connor, 2016b }\end{array}$ \\
\hline $\begin{array}{l}\text { Jerimalai, } \\
\text { Timor-Leste }\end{array}$ & $\begin{array}{l}\text { AMS: }>13,778 \pm 43 \text { BP }(\mathrm{Wk} \\
30504) \\
2 \sigma: 15,746-16,244 \mathrm{cal} . \mathrm{BP}\end{array}$ & $\begin{array}{l}\text { Shaped and ochre stained Nautilus } \\
\text { pompilius shell piece }\end{array}$ & Langley et al., 2016b \\
\hline $\begin{array}{l}\text { Matja Kuru 1, } \\
\text { Timor-Leste }\end{array}$ & $\begin{array}{l}\text { AMS: c. } 9940 \pm 60 \mathrm{BP}(\mathrm{OZF}-784) \\
2 \sigma: 10,584-11,081 \mathrm{cal} . \mathrm{BP}\end{array}$ & $\begin{array}{l}4 \text { Oliva sp. shell beads with ochre } \\
\text { staining }\end{array}$ & $\begin{array}{l}\text { Langley and } \\
\text { O'Connor, } 2016\end{array}$ \\
\hline $\begin{array}{l}\text { Matja Kuru 2, } \\
\text { Timor-Leste }\end{array}$ & $\begin{array}{l}\text { AMS: c. } 9260 \pm 50 \text { BP (OZG-898) } \\
2 \sigma: 9690-10,190 \text { cal. BP }\end{array}$ & $\begin{array}{l}2 \text { Oliva sp. Shell beads with ochre } \\
\text { staining }\end{array}$ & $\begin{array}{l}\text { Langley and } \\
\text { O’Connor, } 2016\end{array}$ \\
\hline
\end{tabular}




\begin{tabular}{|l|l|l|l|}
\hline $\begin{array}{l}\text { Xom Trai, } \\
\text { Vietnam }\end{array}$ & to & $\begin{array}{l}\text { Three basalt blocks with incised linear } \\
\text { decoration and stained with red ochre, } \\
\text { plus two river cobbles with incised } \\
\text { decoration also stained with red ochre. }\end{array}$ & $\begin{array}{l}\text { Rabett and Piper, } \\
2012 ; \text { Nguyen, 2015 }\end{array}$ \\
\hline
\end{tabular}

\subsection{Ornamentation}

While there have been significant finds of perforated animal teeth and shells in north and central China (i.e., at Zhoukoudian and Xiaogushan) (Norton and Jin, 2009), similar items are yet to be reported in the southern region of this vast country. Perforated teeth very similar to those found in Palaeolithic contexts in Eurasia have been found in the Hoabinhian levels of Xom Trai, Vietnam, which produced a $3.2 \mathrm{~cm}$-long mammalian tooth with a small, bifacially carved perforation through its root. This artefact dates to between 22,000 and 19,000 cal. BP (Nguyen, 2000 cited in Rabett and Piper, 2012). At nearby Du Sang, the Hoabinhian levels also produced a perforated mammalian tooth, this one $5.5 \mathrm{~cm}$ long (Nguyen, 2015).

Ornamentation made on hard animal materials have also been recovered from Leang Bulu Bettue, Sulawesi, where two conjoining artefacts dated to around 26,000 cal. BP demonstrated the manufacture of disk-shaped beads in Babirusa incisor. Also found here, was a perforated bear cuscus phalange exhibiting evidence for having been worn as a pendant (Brumm et al., 2017). Items which were clearly pieces of adornment are lacking from Thai pre-Neolithic contexts, though Pookajorn (1996) has reported that both Cypraea sp. and Perna sp. marine shells were recovered from the first cultural level (Hoabinhian) of Sakai Cave located in the Palian district of Trang Province. Dated to 9321-11,211 cal. BP. Although these shells have not been anthropogenically altered, they have been transported far from the sea, leading Pookajorn (1996:207) to argues that they "probably had great value for the inhabitants" and that these "rare type of shells are ornamental items at this site."

Excavations in Timor-Leste are also revealing assemblages of prehistoric ornaments, this time in a range of marine shell species (Nassarius spp., Oliva spp., and Nautilus pompilus) (Langley and O'Connor, 2015, 2016; Langley et al., 2016b). These finds, mentioned above, include fractured pieces of worked and ochre stained Nautilus shell, whole Oliva shell beads, and later (mid-Holocene) Nassarius shell appliqués. Similar finds are reported for a terminal Pleistocene context in Liang Nabulei Lisa (O'Connor et al., 2005a), and several other undated Hoabinhian and Sampung contexts in Indonesia and Timor-Leste (e.g., van Heekeren, 1972; O’Connor, 2010). 
Table 7: Pleistocene examples of ornamentation in Sunda and Wallacea.

\begin{tabular}{|c|c|c|c|}
\hline \multicolumn{4}{|c|}{ Osseous Ornaments } \\
\hline Site & Age & Evidence & Source \\
\hline $\begin{array}{l}\text { Leang Bulu } \\
\text { Bettue, Sulawesi }\end{array}$ & $\begin{array}{l}\text { AMS: } 22,265 \pm 121 \mathrm{BP}(\mathrm{Wk}- \\
42068) \\
2 \sigma: 26,145-26,959 \mathrm{cal} . \mathrm{BP}\end{array}$ & $\begin{array}{l}\text { Two refitting Babyrousa sp. lower incisor } \\
\text { fragments attesting to disc bead manufacture }\end{array}$ & $\begin{array}{l}\text { Brumm et al., } \\
2017\end{array}$ \\
\hline $\begin{array}{l}\text { Leang Bulu } \\
\text { Bettue, Sulawesi }\end{array}$ & $\begin{array}{l}\mathrm{U}: 24.6 \pm 0.2 \mathrm{ka}(605-0274 \\
\text { to } \\
\mathrm{U}: 25.9 \pm 0.7 \mathrm{ka}(605-1900)\end{array}$ & $\begin{array}{l}\text { Perforated Ailurops ursinus phalange long } \\
\text { bone with red and black pigments inside and } \\
\text { on one end (possible blow-pipe). }\end{array}$ & $\begin{array}{l}\text { Brumm et al., } \\
2017\end{array}$ \\
\hline $\begin{array}{l}\text { Xom Trai, } \\
\text { Vietnam }\end{array}$ & $\begin{array}{l}16,130 \pm 90 \mathrm{BP}(\mathrm{B} \ln -3042) \\
\text { to } \\
18,420 \pm 150(\mathrm{~B} \ln -3472)\end{array}$ & $3.2 \mathrm{~cm}$-long mammalian tooth, perforated & $\begin{array}{l}\text { Rabett and Piper, } \\
\text { 2012; Nguyen, } \\
2015\end{array}$ \\
\hline $\begin{array}{l}\text { Du Sang, } \\
\text { Vietnam }\end{array}$ & Hoabinhian (Pleistocene layers) & $5.5 \mathrm{~cm}$-long mammalian tooth, perforated & Nguyen, 2015 \\
\hline $\begin{array}{l}\text { Gua Lawa, } \\
\text { Indonesia }\end{array}$ & Hoabinhian & $\begin{array}{l}\text { Two bored canine teeth, two perforated oval } \\
\text { plates of mother-of-pearl and four fragments of } \\
\text { an 'amulet' of bone incised with a geometric } \\
\text { pattern. Possibly associated with a child and/or } \\
\text { adult burial. }\end{array}$ & $\begin{array}{l}\text { van Heekeren, } \\
1972\end{array}$ \\
\hline \multicolumn{4}{|c|}{ Marine Shell Ornaments } \\
\hline $\begin{array}{l}\text { Jerimalai, } \\
\text { Timor-Leste }\end{array}$ & $\begin{array}{l}\text { AMS: } 35,387 \pm 534 \text { BP (Wk- } \\
\text { 19232) } \\
2 \sigma: 38,432-40,749 \text { cal. BP } \\
\text { to } \\
\text { AMS: } 37,267 \pm 453 \mathrm{BP}(\mathrm{Wk}- \\
17833) \\
2 \sigma: 40,486-42,117 \mathrm{cal} . \mathrm{BP}\end{array}$ & $\begin{array}{l}\text { Two worked and ochre stained pieces of } \\
\text { Nautilus pompilius - likely ornamental. }\end{array}$ & $\begin{array}{l}\text { Langley et al., } \\
2016 \text { b }\end{array}$ \\
\hline $\begin{array}{l}\text { Jerimalai, } \\
\text { Timor-Leste }\end{array}$ & $\begin{array}{l}\text { AMS: } 33,294 \pm 380 \text { BP (ANU- } \\
48106) \\
2 \sigma: 36,042-38,208 \text { cal. BP }\end{array}$ & Oliva sp. shell bead & $\begin{array}{l}\text { Langley and } \\
\text { O’Connor, } 2016\end{array}$ \\
\hline $\begin{array}{l}\text { Jerimalai, } \\
\text { Timor-Leste }\end{array}$ & $\begin{array}{l}\text { AMS: c. } 10,110 \pm 79 \mathrm{BP}(\mathrm{Wk}- \\
\text { 18156) } \\
2 \sigma: 10,726-11,229 \mathrm{cal} \mathrm{BP} \\
\text { to } \\
\text { AMS: c. } 13,658 \pm 91 \mathrm{BP}(\mathrm{Wk}- \\
\text { 19227) }\end{array}$ & $\begin{array}{l}14 \text { Oliva sp. shell beads }-8 \text { with red ochre } \\
\text { staining }\end{array}$ & $\begin{array}{l}\text { Langley and } \\
\text { O’Connor, } 2016\end{array}$ \\
\hline
\end{tabular}




\begin{tabular}{|c|c|c|c|}
\hline & $2 \sigma: 15,413-16,175$ cal. BP & & \\
\hline $\begin{array}{l}\text { Jerimalai, } \\
\text { Timor-Leste }\end{array}$ & $\begin{array}{l}\text { AMS: }>13,778 \pm 43 \text { BP }(\mathrm{Wk} \\
30504) \\
2 \sigma: 15,746-16,244 \text { cal. BP }\end{array}$ & $\begin{array}{l}\text { Shaped and ochre stained Nautilus pompilius } \\
\text { shell piece }\end{array}$ & $\begin{array}{l}\text { Langley et al., } \\
2016 \text { b }\end{array}$ \\
\hline $\begin{array}{l}\text { Matja Kuru 1, } \\
\text { Timor-Leste }\end{array}$ & $\begin{array}{l}\text { AMS: c. } 9940 \pm 60 \text { BP }(\mathrm{OZF}-784) \\
2 \sigma: 10,584-11,081 \mathrm{cal} . \mathrm{BP}\end{array}$ & 7 Oliva sp. shell beads, 4 with ochre staining & $\begin{array}{l}\text { Langley and } \\
\text { O’Connor, } 2016\end{array}$ \\
\hline $\begin{array}{l}\text { Matja Kuru 2, } \\
\text { Timor-Leste }\end{array}$ & $\begin{array}{l}\text { AMS: c. } 9260 \pm 50 \text { BP (OZG-898) } \\
2 \sigma: 9690-10,190 \text { cal. BP }\end{array}$ & 4 Oliva sp. Shell beads, 2 with ochre staining & $\begin{array}{l}\text { Langley and } \\
\text { O’Connor, } 2016\end{array}$ \\
\hline $\begin{array}{l}\text { Saikai Cave, } \\
\text { Thailand }\end{array}$ & $\begin{array}{l}\text { LSC: } 9020 \pm 360 \text { BP (OAEP- } \\
\text { 1371) } \\
2 \sigma: 9321-11,211 \text { cal. BP } \\
\text { to } \\
\text { LSC: } 9280 \pm 180 \text { BP (OAEP- } \\
1371) \\
2 \sigma: 9964-11,153 \mathrm{cal} . \mathrm{BP}\end{array}$ & $\begin{array}{l}\text { Possible ornamental shells (Cypraea sp. and } \\
\text { Perna sp.) }\end{array}$ & Pookajorn, 1996 \\
\hline $\begin{array}{l}\text { Saentis midden } \\
\text { site, Sumatra }\end{array}$ & Hoabinhian & Melo melo shell with pierced hole in columella & $\begin{array}{l}\text { van Heekeren, } \\
1972\end{array}$ \\
\hline $\begin{array}{l}\text { Gua Lawa, } \\
\text { Indonesia }\end{array}$ & Hoabinhian & $\begin{array}{l}\text { Child burial with 'necklace of perforated } \\
\text { shells' of Natica sp. and one Nerita } \\
\text { chamaeleon }\end{array}$ & $\begin{array}{l}\text { van Heekeren, } \\
1972\end{array}$ \\
\hline $\begin{array}{l}\text { Betpuruh Cave, } \\
\text { Indonesia }\end{array}$ & Hoabinhian & $\begin{array}{l}\text { Small disc bead ('small ring with a hole in the } \\
\text { centre made of mother-of-pearl') - possibly } \\
\text { Nautilus sp. }\end{array}$ & $\begin{array}{l}\text { van Heekeren, } \\
\text { 1972; O’Connor, } \\
2010\end{array}$ \\
\hline
\end{tabular}




\subsection{Rock Art}

Indonesia is now the home to the earliest known figurative depictions and hand stencils in the world. This image is a painted depiction of a large ungulate (possibly with a spear shaft protruding from its flank) that has a minimum age of 40,000 at Lubang Jeriji Saléh, Borneo (Aubert et al, 2018). This same site produced similarly early examples of hand stencils (Table 8). At the nearby Maros kasts of Sulawesi, a similarly extensive dating program resulted in the identification of a hand stencil with a minimum age of $36.9 \mathrm{ka}$. A figurative painting of a babirusa at the same site was found to be made at least $44 \mathrm{ka}$ (Aubert et al., 2014). Red pigmented paintings of Pleistocene antiquity have also been identified in Timor-Leste (Aubert et al., 2007).

Table 8: Current evidence for the Pleistocene examples of rock art in Sunda and Wallacea.

\begin{tabular}{|c|c|c|c|}
\hline \multicolumn{4}{|c|}{ Rock Art — Directly Dated } \\
\hline Site & Age & Evidence & Source \\
\hline $\begin{array}{l}\text { Lubang Jeriji } \\
\text { Saléh, Borneo }\end{array}$ & $\begin{array}{l}\mathrm{U}:>26.09 \pm 2.50 \mathrm{ka}(\mathrm{LJS} 2.4) \\
\text { to } \\
\mathrm{U}: \leq 50.28 \pm 1.55 \mathrm{ka}(\mathrm{LJS} 2.5)\end{array}$ & Reddish-orange hand stencil & $\begin{array}{l}\text { Aubert et al., } \\
2018\end{array}$ \\
\hline $\begin{array}{l}\text { Liang Téwét, } \\
\text { Borneo }\end{array}$ & $\mathrm{U}: \leq 103.3 \mathrm{ka}(\mathrm{LT} 1)$ & Reddish-orange hand stencil & $\begin{array}{l}\text { Aubert et al., } \\
2018\end{array}$ \\
\hline $\begin{array}{l}\text { Liang Karim, } \\
\text { Borneo }\end{array}$ & $\mathrm{U}: \leq 82.6 \mathrm{ka}(\mathrm{LK} 1)$ & $\begin{array}{l}\text { Reddish-orange animal painting, possibly a } \\
\text { tapir }\end{array}$ & $\begin{array}{l}\text { Aubert et al., } \\
2018\end{array}$ \\
\hline $\begin{array}{l}\text { Lubang Jeriji } \\
\text { Saléh, Borneo }\end{array}$ & $>39.93 \pm 0.57 \mathrm{ka}(\mathrm{LJS} 1 \mathrm{~A} .3)$ & $\begin{array}{l}\text { Large reddish-orange animal figure, possibly a } \\
\text { Bornean banteng, possibly with a spear shaft } \\
\text { protruding from its flank }\end{array}$ & $\begin{array}{l}\text { Aubert et al., } \\
2018\end{array}$ \\
\hline $\begin{array}{l}\text { Lubang Jeriji } \\
\text { Saléh, Borneo }\end{array}$ & $\begin{array}{l}>38.80 \pm 1.60 \mathrm{ka}(\text { LJS5.4) and } \\
>40.30 \pm 3.10 \mathrm{ka}(\text { LJS6.3) }\end{array}$ & Two reddish-orange hand stencils & $\begin{array}{l}\text { Aubert et al., } \\
2018\end{array}$ \\
\hline $\begin{array}{l}\text { Lubang Jeriji } \\
\text { Saléh, Borneo }\end{array}$ & $\begin{array}{l}\mathrm{U}: \geq 15.64 \pm 0.10 \mathrm{ka}(\mathrm{LJS} 3.7) \\
\text { and } \\
\mathrm{U}: \geq 16.29 \pm 0.09 \mathrm{ka}(\mathrm{LJS} 4.6)\end{array}$ & Hand stencils & $\begin{array}{l}\text { Aubert et al., } \\
2018\end{array}$ \\
\hline $\begin{array}{l}\text { Leang } \\
\text { Timpuseng, } \\
\text { Sulawesi }\end{array}$ & $\begin{array}{l}\mathrm{U}:>36.9+1.60 /-1.50 \mathrm{ka}(\mathrm{LT} 1.2) \\
\text { to } \\
\mathrm{U}: 40.70+0.87 /-0.84 \mathrm{ka}(\mathrm{LT} 2.3)\end{array}$ & Hand stencil & $\begin{array}{l}\text { Aubert et al., } \\
2014\end{array}$ \\
\hline
\end{tabular}




\begin{tabular}{|c|c|c|c|}
\hline $\begin{array}{l}\text { Leang } \\
\text { Timpuseng, } \\
\text { Sulawesi }\end{array}$ & $\begin{array}{l}\mathrm{U}:>36.9+1.60 /-1.50 \mathrm{ka}(\mathrm{LT} 1.2) \\
\text { to } \\
\mathrm{U}: 40.70+0.87 /-0.84 \mathrm{ka}(\mathrm{LT} 2.3)\end{array}$ & $\begin{array}{l}\text { Large naturalistic animal depiction, possibly a } \\
\text { babirusa }\end{array}$ & $\begin{array}{l}\text { Aubert et al., } \\
2014\end{array}$ \\
\hline $\begin{array}{l}\text { Leang Jarie, } \\
\text { Sulawesi }\end{array}$ & $\mathrm{U}:>34.98 \pm 0.41 \mathrm{ka}(\mathrm{LJ} 2)$ & Dark red hand stencil & $\begin{array}{l}\text { Aubert et al., } \\
2014\end{array}$ \\
\hline $\begin{array}{l}\text { Leang } \\
\text { Barugayya 2, } \\
\text { Sulawesi }\end{array}$ & $\begin{array}{l}\mathrm{U}:>44.00 \pm+9.10 /-8.30 \mathrm{ka} \\
\text { (LB4.2) }\end{array}$ & $\begin{array}{l}\text { Large red painting of an indeterminate animal } \\
\text { (probably a pig, a babirusa or Sus celebensis) }\end{array}$ & $\begin{array}{l}\text { Aubert et al., } \\
2014\end{array}$ \\
\hline $\begin{array}{l}\text { Leang } \\
\text { Sampeang, } \\
\text { Sulawesi }\end{array}$ & $\mathrm{U}:>32.60 \pm 0.76 \mathrm{ka}(\mathrm{LS} 1.2)$ & Red hand stencil & $\begin{array}{l}\text { Aubert et al. } \\
2014\end{array}$ \\
\hline $\begin{array}{l}\text { Lene Hara, } \\
\text { Timor-Leste }\end{array}$ & $\begin{array}{l}\mathrm{U}: 24.00 \pm 1.50 \mathrm{ka}(\# 2) \\
\text { to } \\
\mathrm{U}: 29.30 \pm 1.20 \mathrm{ka}(\# 3)\end{array}$ & Red pigmented lamination & $\begin{array}{l}\text { Aubert et al. } \\
2007\end{array}$ \\
\hline $\begin{array}{l}\text { Gua Jing, } \\
\text { Sulawesi }\end{array}$ & $\begin{array}{l}\mathrm{U}:>24.00 \pm 1.10(\mathrm{GJ} 2.2) \\
\text { to } \\
\mathrm{U}: 30.90+1.70 /-1.80 \mathrm{ka}(\mathrm{GJ} 1.3)\end{array}$ & Two hand stencils & $\begin{array}{l}\text { Aubert et al., } \\
2014\end{array}$ \\
\hline $\begin{array}{l}\text { Leang } \\
\text { Barugayya 1, } \\
\text { Sulawesi }\end{array}$ & $\begin{array}{l}\mathrm{U}:>19.70 \pm 1.00(\mathrm{LB} 2.3) \\
\text { to } \\
\mathrm{U:} 29.10 \pm+3.20 /-3.10 \mathrm{ka} \\
\text { (LB1.2) }\end{array}$ & $\begin{array}{l}\text { Two dark mulberry-coloured (almost black) } \\
\text { hand stencils }\end{array}$ & $\begin{array}{l}\text { Aubert et al., } \\
2014\end{array}$ \\
\hline $\begin{array}{l}\text { Liang Banteng, } \\
\text { Borneo }\end{array}$ & $\begin{array}{l}\mathrm{U}: \geq 17.57 \pm 0.10 \mathrm{ka}(\mathrm{LBT} 2.3) \\
\text { and } \\
\mathrm{U}: \geq 19.80 \pm 0.12 \mathrm{ka}(\mathrm{LBT} 1.3)\end{array}$ & $\begin{array}{l}\text { Two mulberry-coloured hand stencils with } \\
\text { internal decorations and tree-like motifs }\end{array}$ & $\begin{array}{l}\text { Aubert et al., } \\
2018\end{array}$ \\
\hline $\begin{array}{l}\text { Leang Lompoa, } \\
\text { Sulawesi }\end{array}$ & $\begin{array}{l}\mathrm{U}:>17.77 \pm 0.42 \text { (LL3.2) } \\
\text { to } \\
\mathrm{U}: 29.30+1.20 /-1.10 \mathrm{ka} \text { (LL2.2) }\end{array}$ & Hand stencils & $\begin{array}{l}\text { Aubert et al., } \\
2014\end{array}$ \\
\hline $\begin{array}{l}\text { Liang Sara, } \\
\text { Borneo }\end{array}$ & $\mathrm{U}:>14.93 \pm 0.36 \mathrm{ka}(\mathrm{LSR} 2.3)$ & Mulberry-coloured hand stencil & $\begin{array}{l}\text { Aubert et al., } \\
2018\end{array}$ \\
\hline $\begin{array}{l}\text { Liang Sara, } \\
\text { Borneo }\end{array}$ & $\mathrm{U}:>13.8 \pm 0.27 \mathrm{ka}(\mathrm{LSR} 1.3)$ & $\begin{array}{l}\text { Mulberry-coloured Datu Saman human figure } \\
\text { wearing large ornate headdress }\end{array}$ & $\begin{array}{l}\text { Aubert et al., } \\
2018\end{array}$ \\
\hline
\end{tabular}




\subsection{Portable Art}

The earliest symbolic evidence for the whole region takes the form of a Pseudodon valve engraved with a geometric zig-zag pattern by Homo erectus some 430,000 years ago (Joordens et al., 2015). Following a long temporal gap, it is not until after modern humans had reached the area that the next examples of portable art are found. First is the red marked turtle plastron (39,880-41,601 cal. BP) from Niah Caves, Borneo (described above). This artefact is the only pre-Neolithic example of 'portable art' reported from Malaysia, Brunei, or the Philippines.

Similarly, there are few cases for portable art on the Southeast Asian mainland, with those best reported being from Vietnam. Xom Trai Cave - mentioned above for colourant instances - also produced from its Hoabinhian levels incised stones: five stones display deliberate and apparently decorative linear incisions on their surfaces (Nguyen, 2015). Three are flat basalt blocks which are partially stained by red ochre, with the first example having 45 short straight parallel incisions across its surface. On this artefact, some of the lines are arranged in a grid like fashion, while others are concentrated in groups. The second stone also exhibits a cross-hatched pattern on one surface, while the third features elongated parallel lines incised down the longitudinal axis of the stone. On this last stone, Nguyen (2015:137) has observed that the overall image "looks like the skeleton of a herring". Weathered red ochre still adheres to one surface of this last artefact, though not the upper surface, where traces of grinding are present. The next find is a tear-drop shaped river cobble, with incised parallel lines arranged in a herringbone pattern covering its surface. At one end of the stone, the surface is polished and stained with red ochre so that the decoration has been partially erased. Finally, an oval-shaped artefact stained with red ochre has its entire upper surface marked by a zig-zag pattern composed of numerous, short, parallel lines. The observed layers of use traces - incised decoration under ochre staining - suggests that a close relationship between the stones and ochre processing existed (Nguyen, 2015). Other incised stones and bone fragments have been reported for Hoabinhian contexts in Vietnam. Colani $(1929,1930)$ described several engravings on stalactites (as parietal art), stones, and pieces of worked bone (portable) from Dong-Noi Cave, My-Tê Cave in Thanh-Hoa province, Na-Ca Cave in Thainguyen province, Len-Dat Cave, and Lam-Gan Cave in Hoa Binh province, which she suggests may depict humans, animals, grasses, and plants.

Most recently, five incised stones were reported for Leang Bulu Bettue, Sulawesi - in this case, on the cortex of tool stone (Brumm et al., 2017). Dated to contexts between 30,000 and 22,000 cal. BP, the engravings are geometric in style, including crosses and oblique lines. 
Table 9: Pleistocene examples of portable art in Sunda and Wallacea.

\begin{tabular}{|c|c|c|c|}
\hline \multicolumn{4}{|l|}{ Portable Art } \\
\hline Site & Age & Evidence & Source \\
\hline Trinil, Java & $>426 \pm 89$ Myr (DUB1006-f(I)) & $\begin{array}{l}\text { Pseudodon vondembuschianus trinilensis valve } \\
\text { (DUB1006-fL) incised with a geometric } \\
\text { engraving }\end{array}$ & $\begin{array}{l}\text { Joordens et al., } \\
2015\end{array}$ \\
\hline $\begin{array}{l}\text { Niah Caves, } \\
\text { Sarawak }\end{array}$ & $\begin{array}{l}\text { AMS: } 35,890 \pm 250 \mathrm{BP}(\mathrm{OxA}- \\
15163) \\
2 \sigma: 39,931-41,162 \mathrm{cal} . \mathrm{BP} \\
\text { to } \\
\text { AMS: } 36,470 \pm 250 \mathrm{BP}(\mathrm{OxA}- \\
15164) \\
2 \sigma: 40,526-41,635 \mathrm{cal} . \mathrm{BP}\end{array}$ & $\begin{array}{l}\text { Fragment of hard-shelled turtle plastron } \\
\text { displaying a pigmented section with a straight } \\
\text { and clearly defined perimeter }\end{array}$ & $\begin{array}{l}\text { Reynolds et al., } \\
2013\end{array}$ \\
\hline $\begin{array}{l}\text { Leang Bulu } \\
\text { Bettue, Sulawesi }\end{array}$ & $\begin{array}{l}\text { AMS: } 18,126 \pm 51 \mathrm{BP}(\mathrm{Wk}- \\
37742) \\
2 \sigma: 21,792-22,196 \mathrm{cal} . \mathrm{BP} \\
\text { to } \\
\text { AMS: } 22,265 \pm 121 \mathrm{BP}(\mathrm{Wk}- \\
42068) \\
2 \sigma: 26,145-26,959 \text { cal. BP }\end{array}$ & $\begin{array}{l}\text { Five stone artefacts with geometric patterns } \\
\text { incised into their cortex }\end{array}$ & $\begin{array}{l}\text { Brumm et al., } \\
2017\end{array}$ \\
\hline $\begin{array}{l}\text { Xom Trai, } \\
\text { Vietnam }\end{array}$ & $\begin{array}{l}16,130 \pm 90 \mathrm{BP}(\mathrm{B} \ln -3042) \\
\text { to } \\
18,420 \pm 150(\mathrm{~B} \ln -3472)\end{array}$ & $\begin{array}{l}\text { Three basalt blocks with incised decoration and } \\
\text { stained with red ochre, plus two river cobbles } \\
\text { with incised decoration also stained with red } \\
\text { ochre }\end{array}$ & $\begin{array}{l}\text { Rabett and Piper, } \\
2012 ; \text { Ngyuen, } \\
2015\end{array}$ \\
\hline $\begin{array}{l}\text { Len-Dat Cave, } \\
\text { Vietnam }\end{array}$ & Hoabinhian & Incised schist - geometric motif & Colani, 1929 \\
\hline $\begin{array}{l}\text { Lam-Gan Cave, } \\
\text { Vietnam }\end{array}$ & Hoabinhian & $\begin{array}{l}\text { Incised bone awl with possible incised plant } \\
\text { motif }\end{array}$ & Colani, 1929 \\
\hline $\begin{array}{l}\text { Dong-Noi Cave, } \\
\text { Vietnam }\end{array}$ & Hoabinhian & Bone bearing engraving & Colani, 1929 \\
\hline $\begin{array}{l}\text { My-Tê Cave, } \\
\text { Vietnam }\end{array}$ & Hoabinhian & Bone bearing engraving & Colani, 1929 \\
\hline $\begin{array}{l}\text { Na-Ca Cave, } \\
\text { Vietnam }\end{array}$ & Hoabinhian & Bone bearing engraving & Colani, 1929 \\
\hline
\end{tabular}




\subsection{Burial}

Early modern human skeletal remains are rare across Southeast Asia before the Neolithic making the identification of the first arrival of our species into the area difficult. Indeed, the only burials clearly identified as having originated from the Pleistocene comes from Xom Trai and Du Sang Rock Shelter, both in Vietnam. At Xom Trai a robust male (08XT-M01) was discovered near the cave mouth, laid with the hip and knee joints flexed. Nguyen (2015) reports that an Antimelania shell sample from the same layer of this burial produced an uncalibrated age of 17,100 \pm 545 BP (HNK-530). At Du Sang, incomplete remains of an individual (04DS-M2) - perhaps indicating a secondary burial deposit — were found (Nguyen 2015). Other than these two instances, only isolated skeletal fragments are reported (e.g., Curnoe et al., 2018, Demeter et al., 2012; O'Connor and Langley, 2018; Westaway et al., 2018), including several (and which include grave goods) from early Holocene (e.g., O'Connor et al., 2005a; Lewis et al., 2008) and poorly dated Hoabinhian contexts (van Heekeren, 1972).

\section{Discussion: Comparisons and Implications}

Pleistocene Sahul, Sunda, and Wallacea have often been depicted as simple and unchanging societies, particularly in comparison to what are seen as the innovative and dynamic populations of Palaeolithic Eurasia and Middle Stone Age Africa. Indeed, O'Connell and Allen (2007:404) concluded that 'in short, it [Sahul's Pleistocene archaeological record] does not appear to be the product of modern human behaviour as such products are conventionally defined' calling into question why then current paradigms were problematic for defining 'cognitive modernity'. Explanations for why the Pleistocene economic and social technologies of Sahul and Sunda were 'crude and rather colourless' found support in the theorised loss of "several technological features (such as fishing, bone tools, and other cultural elements)" owing to "repeated, successive, and cumulative small-scale founder effects" (Mellars, 2006:799). These lost cultural traits (i.e., parietal art, portable art, bone technology, etc.) were then suggested to be essentially 're-invented' and reintroduced into the repertoire of Australian material culture over the succeeding tens of thousands of years (Franklin and Habgood, 2007; Habgood and Franklin, 2008).

Sahul continues to dominate our knowledge of these regions in terms of numbers of Pleistocene sites which have produced evidence of a symbolic nature ( $n=56$ versus $n=14$ in Wallacea and $n=17$ in Sunda). Having said this, recent research efforts in Wallacea by several archaeological teams have yielded significant new evidence for symbolic behavior on a scale somewhat richer than that of Sahul. Wallacea likewise appears also to outrank Sunda in terms of the richness of symbolic evidence (Aubert et al., 2018). Apart from the recent rock art findings, benefiting from new techniques and advances in the field of rock art dating - and which might be expected to follow in Australia over the coming years - Wallacea has been producing significant numbers (several hundred) of marine shell ornaments. The greatest quantity of shell beads found at a single site located in Sahul is 22 Conus sp. at Mandu Mandu in Western Australia, though most sites produced only one (see Table 2). This difference in artefact richness between Sahul and Wallacea may be explained by the disappearance of coastal sites on Sahul with the incursion of the coast after the LGM. The steep coast lines of Wallacea, on the other hand, have preserved the earliest coastal sites.

As it stands, the only category of symbolic evidence which continues to be entirely absent from the whole region is portable pieces of figurative art (Figure 2). Such items have played a large role in narrative building around Palaeolithic Eurasian communities and their social sophistication (e.g., Barton et al., 1994; Conard, 2003, 2009), and their absence in Australia and Southeast Asia has often been noted (see above). 


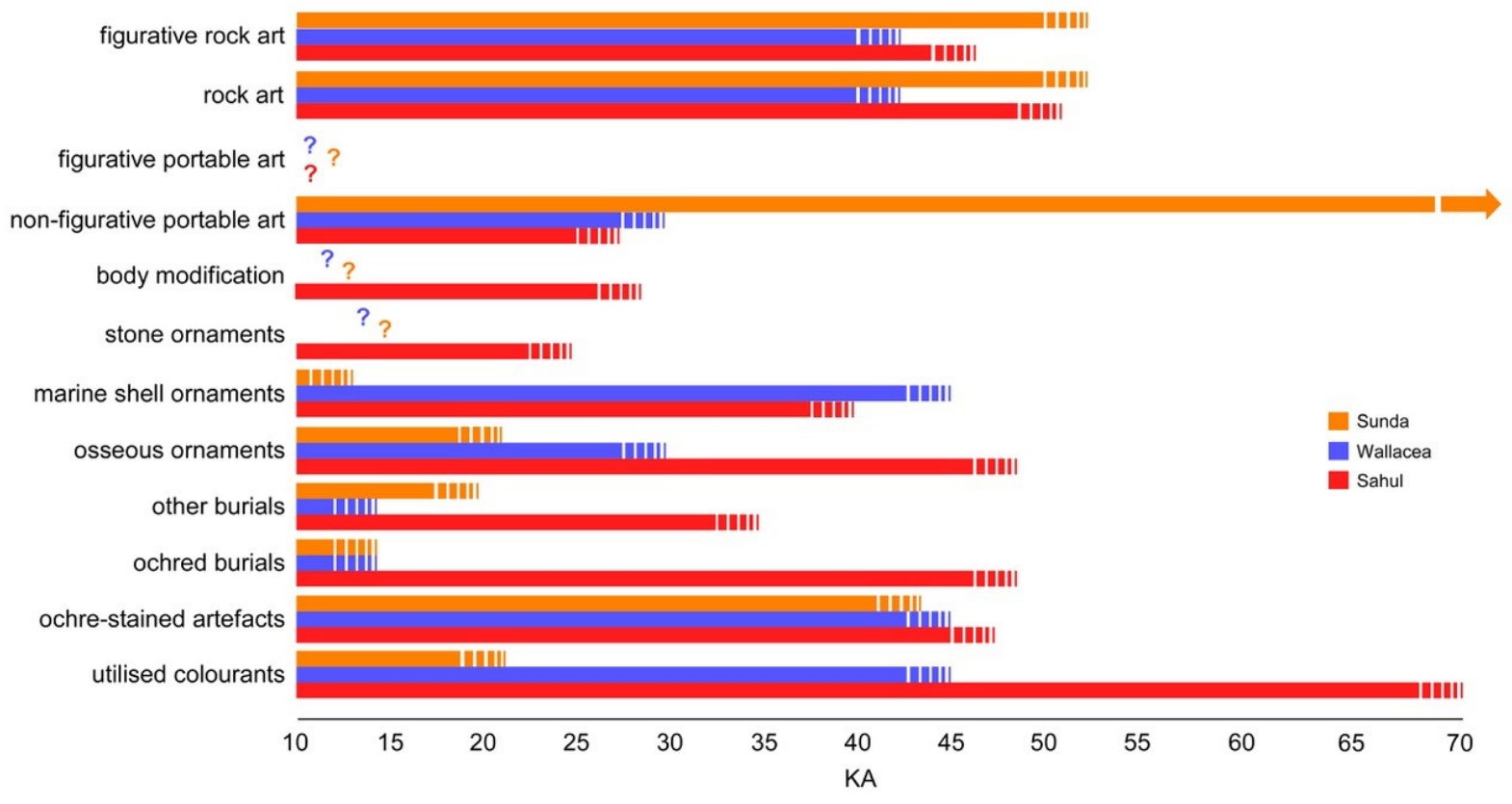

Figure 2: Temporal distribution of types of symbolic behaviour found in Sunda, Wallacea and Sahul.

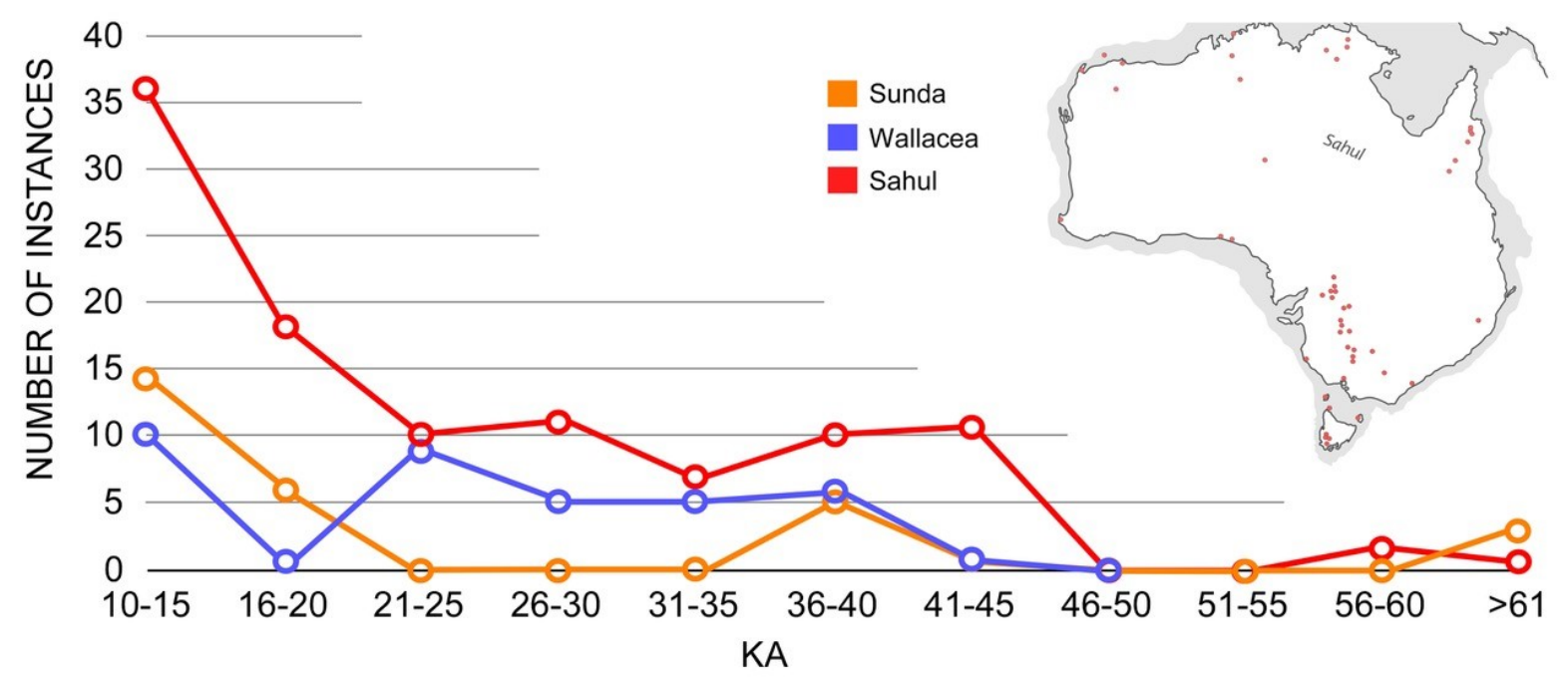

Figure 3: Temporal distribution of instances of symbolic behaviour in Sunda, Wallacea and Sahul. 
When the currently known instances of symbolic behaviours of Sunda and Sahul are plotted against each other (Figure 3), there appears to be some synchronicity between regions. Though this pattern may simply represent taphonomic processes, it may also reflect a real trend in the archaeological record, tracking increasing populations and a growing need for increased social signalling (see Langley et al., 2011; Williams, 2013; Williams et al., 2013; Bradshaw et al., 2019). Also of interest is that whereas some 10 years ago Sahul offered the earliest instances for symbolic behaviour in the region, Sunda and Wallacea have now surpassed Sahul with evidence reaching back tens to hundreds of thousands of years before examples from Sahul (Figure 2). Similarly, evidence for other types of complex behaviours have been drawn from the Sunda and Wallacean archaeological record which pre-date examples in Australia, for example fishhooks at c.23,000-16,000 cal. BP and pelagic fishing from 42,000 cal. BP (O’Connor et al., 2011). Again, this could simply be an artefact of taphonomy given the drowning of the broad coastal plain around the margin of Sahul and the general absence of limestone shelters (Williams et al., 2018).

While we have focused on symbolic evidence in this paper, our earlier review also included economic and technological behaviours. We note here that the inventory of technologies and economic behaviours has also increased in the last decade, though we have not included these in our tables above since they are far fewer in number. Most notable is the evidence from Madjedbebe for seed grinding, point production, diverse plant food collection, and the production of edge ground axes from the earliest phase of human occupation at c.65,000 years ago (Clarkson et al., 2017). These are now the oldest known examples of hafted edge ground axes in the world (followed by ages of 50,000 years ago from the Kimberley and 35,000 years ago from central Arnhem Land, Hiscock et al., 2016; Geneste et al., 2010), and the oldest known examples of seed grinding outside of Africa. Hafting of backed artefacts has been identified at Karnatukul (previously Serpent's Glen) at 43,330 cal. BP (McDonald et al., 2018), and many younger examples found at Warratyi at 38,725-40,946 cal. BP, Ngarrabullgin at >37,170-c.30,000 (Fullagar and David, 1997), OLH at 15,155 cal. BP (Slack et al., 2004), and Shaws Creek KII at 14,700 BP (Nanson et al., 1987). Stone points, presumably used as projectile tips and knives, were hitherto thought only to occur in the mid-Holocene in Australia but now appear to also occur between 65,000-53,000 years ago (Clarkson et al., 2017). Northern Australian sites have also yielded new insights into early coastal economies, with evidence of shellfish gathering taking place on the northwest Pilbara coast by 42,500 years ago (Veth et al., 2017). Increased sample size and reexamination of the oldest sites has thus greatly extended the antiquity of unique and complex technologies as well as built new understandings of early economic developments in the region. We foresee continued new insights emerging into the early technology and economy of the Sunda, Sahul, and Wallacean regions as the pace of archaeological research in these regions continues to intensify.

\section{Conclusion}

Long-held notions regarding the dominant role of Western Europe (in particular) in the evolution of human cultural innovation must be revised in light of new discoveries made in the Far East and Pacific. The abundant early traces of art, burial and personal ornamentation found across Sunda, Sahul, and Wallacea now places the archaeological record of early modern humans in these regions on an equal footing with those of Eurasia and Africa. Above all, the global archaeological record of early modern humans demonstrates beyond doubt that symbolic as well as complex economic and technological capabilities were shared by all modern humans wherever they lived and in whatever environments they inhabited. The inbuilt human drive to innovate, represent, harvest, and modify our environment is attested in all regions of the earth from the earliest inception of our species. In regions until now poorly explored, and through sustained and increasing effort, archaeology has at last begun to deliver on its promise to uncover the rich, diverse and innovative past shared by our own species worldwide. 
As we anticipated several years ago, increasing sample sizes have dramatically enlarged symbolic inventories in the region (Langley et al., 2011). In another ten years it will be interesting to see what - if any - differences in symbolic material culture are still missing from Sahul, Sunda, and Wallacea. As predicted, increased sample size, methodological improvements in recovery, dating and material identification, and above all sustained effort (Hiscock, 2017), are all fundamental in increasing the rate of discovery of rare sites and finds. While we might follow Hiscock (2017) in predicting that rates of discovery of new kinds of evidence will eventually flatten out, for the time being we predict that the rate of discovery will increase in these poorly known regions where the pace of archaeological research is intensifying.

\section{Acknowledgements}

Parts of this research was supported by the Australian Research Council DECRA grant DE170101076 awarded to MCL. CC is the recipient of an Australian Research Council Future Fellowship (project FT160100242). SU is the recipient of an Australian Research Council Future Fellowship (project FT120100656). This research was conducted by the Australian Research Council Centre of Excellence for Australian Biodiversity and Heritage (project number CE170100015). Thank-you to Richard Cosgrove, Kathleen Dabell, Bruno David, Kane Ditchfield, Charles Dortch, Fiona Hook, Zenobia Jacobs, Matthew Leavesley, Jo McDonald, Fiona Petchey, Philip Piper, Daniel Rosendahl, Michael Slack, Sarah Slater and Mike Smith for assistance and advice.

\section{References}

Allen, H., 1972. Where the Crow Flies Backwards: Man and Land in the Darling Basin. Unpublished PhD thesis, Australian National University.

Allen, J., 1996. Warreen cave, in: Allen, J., (Ed.), Report of the Southern Forests Archaeological Project: Site Descriptions, Stratigraphies and Chronologies. School of Archaeology, La Trobe University, Bundoora, pp. 135-167.

Allen, J., Marshall, B., Ranson, D., 1989. A note on excavations at the Maxwell River site, M86/2, southwest Tasmania. Aust. Archaeol. 29, 3-8.

Antón, S.C., Weinstein, K.J., 1999. Artificial cranial deformation and fossil Australians revisited. J. Hum. Evol. 36(2), 195-209. https://doi-org.elibrary.jcu.edu.au/10.1006/jhev.1998.0266

Aubert, M., 2012. A review of rock art dating in the Kimberley, Western Australia. J. Archaeol. Sci. 39(3), 573-577. https://doi.org/10.1016/j.jas.2011.11.009

Aubert, M., Brumm, A., Ramli, M., Sutikna, T., Saptomo, E.W., Hakim, B., Morwood, M.J., van den Bergh, G.D., Kinsley, L., Dosseto, A., 2014. Pleistocene cave art from Sulawesi, Indonesia. Nature 514, $223-227$. https://doi.org/10.1038/nature13422

Aubert, M., O’Connor, S., McCulloch, M., Mortimer, G., Watchman, A., Richer-LaFlèche, M., 2007. Uranium-series dating rock art in East Timor. J. Archaeol. Sci. 34(6), 991-996.

https://doi.org/10.1016/j.jas.2006.09.017 
Aubert, M., Setiawan, P., Oktaviana, A.A., Brumm, A., Sulistyarto, P.H., Saptomo, E.W., Istiawan, B., Ma'rifat, T.A., Wahyuono, V.N., Atmoko, F.T., Zhao, J-x., Huntley, J., Taçon, P.S.C., Howard, D.L., Brand, H.E.A., 2018. Palaeolithic cave art in Borneo. Nature 564, 254-257. https://doi.org/10.1038/s41586-0180679-9

Balme, J., 2000. Excavations revealing 40,000 years of occupation at Mimbi Caves, south central Kimberley, Western Australia. Aust. Archaeol. 51, 1-5. https://doi.org/10.1080/03122417.2000.11681674

Balme, J., Davidson, I., McDonald, J., Stern, N., Veth, P., 2009. Symbolic behaviour and the peopling of the southern arc route to Australia. Quat. Int. 202(1-2), 59-68. https://doi.org/10.1016/j.quaint.2008.10.002

Balme, J., Morse, K. 2006. Shell beads and social behaviour in Pleistocene Australia. Antiquity 80, 799-811. https://doi.org/10.1017/S0003598X00094436

Balme, J., O'Connor, S., Langley, M.C., 2018. Marine shell ornaments in northwestern Australian archaeological sites: different meanings over time and space, in: Langley, M.C., Litster, M., Wright, D., May, S.K., (Eds), The Archaeology of Portable Art: Southeast Asian, Pacific, and Australian Perspectives. Routledge, Oxon, pp. 258-273.

Barth, F. (Ed.), 1969. Ethnic Groups and Boundaries: The Social Organization of Culture Difference. Allen \& Unwin, London.

Barton, C.M., Clark, G.A., Cohen, A.E., 1994. Art as information: explaining Upper Palaeolithic art in Western Europe. World Archaeol. 26(2), 185-207. https://doi.org/10.1080/00438243.1994.9980272

Beck, W., Donahue, D.J., Jull, A.J.T., Burr, G., Broecker, W.S., Bonani, G., Hajdas, I., Malotki, E., 1998. Ambiguities in direct dating of rock surfaces using radiocarbon measurements. Science 280 (5372), 2132 2139. https://doi.org/10.1126/science.280.5372.2132

Bednarik, R.G., 1986. Cave use by Australian Pleistocene man. Proc. Univ. Bristol Spelaeol. Soc. 17(3), 227-245.

Bednarik, R.G., 1994. The Pleistocene art of Asia. J. World Prehist. 8(4), 351-375.

https://doi.org/10.1007/BF02221090

Bednarik, R.G., 1998. Microscopic analysis of 'engraved plaques' and other objects from Devil's Lair. J. Royal Soc. West. Aust. 81, 165-175.

Bednarik, R.G., 1999. The speleothem medium of finger flutings and its isotopic geochemistry. The Artefact 22, 49-64.

Bird, M.I. Beaman, R.J., Condie, S.A., Cooper, A., Ulm, S., Veth, P., 2018 Palaeogeography and voyage modeling indicates early human colonization of Australia was likely from Timor-Roti. Quat. Sci. Rev. 191, 431-439. https://doi.org/10.1016/j.quascirev.2018.04.027

Bird, M.I., Condie, S.A., O’Connor, S., O’Grady, D., Reepmeyer, C., Ulm, S., Zega, M., Saltré, F., Bradshaw, C.J.A., 2019. Early human settlement of Sahul was not an accident. Scientific Reports 9, 8220. https://doi.org/10.1038/s41598-019-42946-9

Bowdler, S., 1984. Hunter Hill, Hunter Island: Archaeological Investigations of a Prehistoric Tasmanian Site. Terra Australis 8. Department of Prehistory, Research School of Pacific Studies, Australian National University, Canberra. 
Bowdler, S., 1993. Sunda and Sahul: A 30 kyr culture area?, in: Smith, M.A., Spriggs, M., Fankhauser, B., (Eds), Sahul in Review: Pleistocene Archaeology in Australia, New Guinea and Island Melanesia.

Occasional Papers in Prehistory 24. Department of Prehistory, Research School of Pacific Studies, Australian National University, Canberra, pp. 60-70.

Bowler, J.M., 1983. Lunettes as indices of hydrologic change: a review of Australian evidence. Proc. R. Soc. Vic. 95, 147-168.

Bowler, J.M., 1998. Willandra Lakes revisited: environmental framework for human occupation. Archaeol. in Oceania 33(3), 120-155. https://doi.org/10.1002/j.1834-4453.1998.tb00414.x

Bowler, J.M., Johnston, H., Olley, J.M., Prescott, J.R., Roberts, R.G., Shawcross, W., Spooner, N.A., 2003. New ages for human occupation and climatic change at Lake Mungo, Australia. Nature 421, 837-840. https://doi.org/10.1038/nature01383

Bowler, J.M., Jones, R., Allen, H., Thorne, A.G., 1970. Pleistocene human remains from Australia: a living site and human cremation from Lake Mungo, western New South Wales. World Archaeol. 2(1), 39-60. https://doi.org/10.1080/00438243.1970.9979463

Bowler, J.M., Thorne, A.G., Polach, H.A., 1972. Pleistocene man in Australia: age and significance of the Mungo skeleton. Nature 240, 48-50. https://doi.org/10.1038/240048a0

Bradshaw, C.J.A., Ulm, S., Williams, A.N., Bird, M.I., Roberts, R.G., Jacobs, Z., Laviano, F., Weyrich, L.S., Friedrich, T., Norman, K., Saltré, F., 2019 Minimum founding populations for the first peopling of Sahul. Nature Ecology \& Evolution 3(7), 1057-1063. https://doi.org/10.1038/s41559-019-0902-6

Brain, R., 1979. The Decorated Body. Hutchinson, London.

Bronk Ramsey C., 2009. Bayesian analysis of radiocarbon dates. Radiocarbon 51(1), 337-360. https://doi.org/10.1017/S0033822200033865

Brown, P., 1987. Pleistocene homogeneity and Holocene size reduction: the Australian human skeletal evidence. Archaeol. in Oceania 22(2), 41-67. https://doi.org/10.1002/j.1834-4453.1987.tb00166.x

Brown, P., 1989. Coobool Creek: A Morphological and Metrical Analysis of the Crania, Mandibles and Dentitions of a Prehistoric Australian Human Population. Terra Australis 13. Department of Prehistory, Research School of Pacific Studies, Australian National University, Canberra.

Brown, S., 1993. Mannalargenna Cave: A Pleistocene site in Bass Strait, in: Smith, M.A., Spriggs, M., Fankhauser, B., (Eds), Sahul in Review: Pleistocene Archaeology in Australia, New Guinea and Island Melanesia. Occasional Papers in Prehistory 24. Department of Prehistory, Research School of Pacific Studies, Australian National University, Canberra, pp. pp. 258-274.

Brumm, A., Langley, M.C., Moore, M.W., Hakim, B., Ramli, M., Sumantri, I., Burhan, B., Saiful, A.M., Siagian, L., Suryatman, Sardi, R., Jusdi, A., Abdullah, Mubarak, A.P., Hasliana, Hasrianti, Oktaviana, A.A., Adhityatama, S., van den Bergh, G.D., Aubert, M., Zhao, J.-x., Huntley, J., Li, B., Roberts, R.G., Saptomo, E.W., Perston, Y., Grün, R., 2017. Early human symbolic behavior in the late Pleistocene of Wallacea. Proc. Nat. Acad. Sci. 114(16), 4105-4110. https://doi.org/10.1073/pnas.1619013114

Brumm, A., Moore, M.W., 2005. Symbolic revolutions and the Australian archaeological record. Camb. Archaeol. J. 15(2), 157-175. https://doi.org/10.1017/S0959774305000089 
Bulbeck, D., 2005. The Last Glacial Maximum human burial from Liang Lemdubu in northern Sahulland, in: O'Connor, S., Spriggs, M., Veth, P., (Eds), The Archaeology of the Aru Islands, Eastern Indonesia. Terra Australis 22. ANU E Press, Canberra, pp. 255-294.

Bulbeck, D., Sumantri, I., Hiscock, P., 2004. Leang Sakapao 1, a second dated Pleistocene site from South Sulawesi, Indonesia. Mod. Quat. Res. Southeast Asia 18, 111-128.

Campbell, J., Cole, N., Hatte, E., Tuniz, C., Watchman, A., 1996. Dating of rock surface accretions with Aboriginal paintings and engravings in North Queensland, in: Ulm, S., Lilley, I., Ross, A., (Eds), Australian Archaeology '95: Proceedings of the 1995 Australian Archaeological Association Annual Conference. Tempus 6. Anthropology Museum, Department of Anthropology and Sociology, University of Queensland, St Lucia, QLD, pp. 231-239.

Cane, S., 2001. The great flood: Eustatic change and cultural change in Australia during the late Pleistocene and Holocene, in: Anderson, A., Lilley, I., O'Connor, S., (Eds), Histories of Old Ages: Essays in Honour of Rhys Jones. Pandanus Books, Canberra, pp. 141-166.

Carr, C., 1995. Building a unified middle-range theory of artifact design: historical perspectives and tactics, in: Carr, C., Neitzel, J.E., (Eds.), Style, Society, and Person: Archaeological and Ethnological Perspectives. Springer, Boston, pp. 151-170. https://doi.org/10.1007/978-1-4899-1097-4_6

Chase, P.G., Dibble, H.L., 1987. Middle Paleolithic symbolism: a review of current evidence and interpretations. J. Anthropol. Archaeol. 6(3), 263-296. https://doi.org/10.1016/0278-4165(87)90003-1

Chippindale, C., Taçon, P.S.C., 1998. The Archaeology of Rock-Art. Cambridge University Press, Cambridge.

Chitkament, T., Gaillard, C., Shoocongdej, R., 2016. Tham Lod rockshelter (Pang Mapha district, northwestern Thailand): evolution of the lithic assemblages during the late Pleistocene. Quat. Int. 416, 151-161. https://doi.org/10.1016/j.quaint.2015.10.058

Clark, P., Hope, J., 1985. Aboriginal burials and shell middens at Snaggy Bend and other sites on the central Murray River. Aust. Archaeol. 20, 68-89.

Clarkson, C., Jacobs, Z., Marwick, B., Fullagar, R., Walllis, L., Smith, M., Roberts, R.G., Hayes, E., Lowe, K., Carah, X., Florin, S.A., McNeil, J., Cox, D., Arnold, L.J., Hua, Q., Huntley, J., Brand, H.E.A., Manne, T., Fairbairn, A., Shulmeister, J., Lyle, L., Salinas, M., Page, M., Connell, K., Park, G., Norman, K., Murphy, T., Pardoe, C. 2017. Human occupation of northern Australia by 65,000 years ago. Nature 547, 306-310. https://doi.org/10.1038/nature22968

Clarkson, C., Roberts, R.G., Jacobs, Z., Marwick, B., Fullagar, R., Arnold, L.J., Hua, Q., 2018. Reply to comments on Clarkson et al. (2017) 'human occupation of northern Australia by 65,000 years ago'. Aust. Archaeol. 84(1), 84-89. https://doi.org/10.1080/03122417.2018.1462884

Clarkson, C., Smith, M., Marwick, B., Fullagar, R., Wallis, L.A., Faulkner, P., Manne, T., Hayes, E., Roberts, R.G., Jacobs, Z., Carah, X., Lowe, K.M., Matthews, J., Florin, S.A., 2015. The archaeology, chronology and stratigraphy of Madjedbebe (Malakunanja II): a site in northern Australia with early occupation. J. Hum. Evol. 83, 46-64. https://doi.org/10.1016/j.jhevol.2015.03.014

Clegg, J., 1987. Style and tradition at Sturt's Meadows. World Archaeol. 19(2), 236-255.

https://doi.org/10.1080/00438243.1987.9980037 
Colani, M., 1929. Gravures primitives sur pierre et sur os (stations haobinhiennes et bacsoniennes). Bull. l'Ecole Française d'Extrême-Orient 29, 273-287.

Colani, M., 1930. The Megaliths of Upper Laos.

Cole, N.A., Watchman, A., Morwood, M.J., 1995. Chronology of Laura rock art, in: Morwood, M.J., Hobbs, D.R., (Eds.), Quinkan Prehistory: The Archaeology of Aboriginal Art in S.E. Cape York Peninsula, Australia. Tempus 3. Anthropology Museum, University of Queensland, St Lucia, pp. 147-160.

Conard, N.J., 2003. Palaeolithic ivory sculptures from southwestern Germany and the origins of figurative art. Nature 426, 830-832. https://doi.org/10.1038/nature02186

Conard, N.J., 2008. A critical view of the evidence for a southern African origin of behavioural modernity. South African Archaeol. Soc. Goodwin Ser. 10, 175-179.

Conard, N.J., 2009. A female figurine from the basal Aurignacian of Hohle Fels Cave in southwestern Germany. Nature 459, 248-252. https://doi.org/10.1038/nature07995

Conkey, M.W., 1980. The identification of prehistoric hunter-gatherer aggregation sites: the case of Altamira. Curr. Anthropol. 21(5), 609-630. https://doi.org/10.1086/202540

Cordwell, J.M., Schwarz, R.A., 1979. The Fabrics of Culture: The Anthropology of Clothing and Adornment. Mouton, Paris.

Cosgrove, R., Pike-Tay, A., 2004. The middle Palaeolithic and late Pleistocene Tasmania hunting behaviour: a reconsideration of the attributes of modern human behaviour. Int. J. Osteoarchaeol. 14(3-4), 321-332. https://doi.org/10.1002/oa.764

Cox, D., 2013. Haematite 'Crayons' at Madjedbebe: Drawing Conclusions of Cognitive Modernity. Unpublished BA (Honours) thesis, University of Queensland, Brisbane.

Curnoe, D., Datan, I., Zhao, J.-x., Ung, C.L.M., Aubert, M., Sauffi, M.S., Mei, G.H., Mendoza, R., Taçon, P.S.C., 2018. Rare late Pleistocene-early Holocene human mandibles from the Niah Caves (Sarawak, Borneo). PLOS ONE 13(6), e0196633. https://doi.org/10.1371/journal.pone.0196633

David, B., 1991. Fern Cave, rock art and social formations: rock art regionalisation and demographic models in southeastern Cape York Peninsula. Archaeol. in Ocean. 26(2), 41-57. https://doi.org/10.1002/j.18344453.1991.tb00263.x

David, B., Armitage, R.A., Hyman, M., Rowe, M.W., Lawson, E., 1999 How old is north Queensland's rock-art? A review of the evidence, with new AMS determinations. Archaeol. in Oceania 34(3), 103-120. https://doi.org/10.1002/j.1834-4453.1999.tb00441.x

David, B., Barker, B., Petchey, F., Delannoy, J.-J., Geneste, J.-M., Rowe, C., Eccleston, M., Lamb, L., Whear, R., 2013b. A 28,000 year old excavated painted rock from Nawarla Gabarnmang, northern Australia. J. Archaeol. Sci. 40(5), 2493-2501. https://doi.org/10.1016/j.jas.2012.08.015

David, B., Delannoy, J-J., Mialanes, J., Clarkson, C., Petchey, F., Geneste, J-M., Manne, T., Bird, M.I., Barker, B., Richards, T., Chalmin, E., Castets, G., 2019. 45,610-52,160 years of site and landscape occupation at Nawarla Gabarnmang, Arnhem Land plateau (northern Australia). Quat. Sci. Rev. 215, 64-85. https://doi.org/10.1016/j.quascirev.2019.04.027 
David, B., Geneste, J.-M., Petchey, F., Delannoy, J.-J., Barker, B., Eccleston, M., 2013a. How old are Australia's pictographs? A review of rock art dating. J. Archaeol. Sci. 40(1), 3-10.

https://doi.org/10.1016/j.jas.2012.08.019

David, B., Roberts, R.G., Magee, J., Mialanes, J., Turney, C., Bird, M., White, C., Fifield, L.K., Tibby, J., 2007. Sediment mixing at Nonda Rock: investigations of stratigraphic integrity at an early archaeological site in northern Australia and implications for the human colonisation of the continent. J. Quat. Sci. 22(5), 449479. https://doi.org/10.1002/jqs.1136

Davidson, I., 2003. 'Part and parcel' - blade industries and modern human behaviour. Aust. Archaeol. 57, 54-63. https://doi.org/10.1080/03122417.2003.11681762

Davidson, I., 2007. Tasmanian Aborigines and the origins of language, in: Mulvaney, J., Tyndale-Biscoe, H., (Eds), Rediscovering Recherche Bay. Academy of the Social Sciences in Australia, Canberra, pp.69-85.

Davidson, I., Noble, W., 1992. Why the first colonisation of the Australian region is the earliest evidence of modern human behaviour. Archaeol. in Oceania 27(3), 135-142. https://doi.org/10.1002/j.1834-

4453.1992.tb00297.x

Dennell, R., Porr, M., (Eds.), Southern Asia, Australia and the Search for Human Origins. Cambridge University Press, Cambridge.

Demeter, F., Shackelford, L.L., Bacon, A.-M., Duringer, P., Westaway, K., Sayavongkhamdy, T., Braga, J., Sichanthongtip, P., Khamdalavong, P., Ponche, J.-L., Wang, H., Lundstrom, C., Patole-Edoumba, E., Karpoff, A.-M. 2012. Anatomically modern human in Southeast Asia (Laos) by 46 ka. Proc. Nat. Acad. Sci. USA 109, 14375-14380. https://doi.org/10.1073/pnas.1208104109

d'Errico, F., 1998. Palaeolithic Origins of Artificial Memory Systems: an Evolutionary Perspective, in: Renfrew, C., Scarre, C., (Eds), Cognition and Material Culture: The Archaeology of Symbolic Storage. McDonald Institute for Archaeological Research, University of Cambridge, Cambridge, pp. 19-50.

d'Errico, F., 2003. The invisible frontier: a multiple species model for the origin of behavioral modernity. Evol. Anthropol. 12(4), 188-202. https://doi.org/10.1002/evan.10113

d'Errico, F., Henshilwood, C., Lawson, G., Vanhaeren, M., Tillier, A.-M., Soressi, M., Bresson, F., Maureille, B., Nowell, A., Lakarra, J., Backwell, L., Julien, M., 2003. Archaeological evidence for the emergence of language, symbolism, and music - an alternative multidisciplinary perspective. J. World Prehist. 17(1), 1-70. https://doi.org/10.1023/A:1023980201043

Donald, M., 1991. Origins of Modern Mind: Three Stages in the Evolution of Culture and Cognition. Harvard University Press, Cambridge.

Dorn, R.I., Clarkson, P.B., Nobbs, M.F., Loendort, L.L., Whitley, D.S., 1992. New approach to the radiocarbon dating of rock varnish, with examples from drylands. Ann. Assoc. Am. Geographers 82(1), 136 151. https://doi.org/10.1111/j.1467-8306.1992.tb01902.x

Dorn, R.I., Nobbs, M., 1992. Further support for the antiquity of South Australian rock engravings. Aust. Aboriginal Stud. 1, 56-60.

Dortch, C.E., 1976. Two engraved stone plaques of late Pleistocene age from Devil's Lair, Western Australia. Archaeol. Phys. Anthropol. in Oceania 11(1), 32-44. https://doi.org/10.1002/j.1834-

4453.1976.tb00233.x 
Dortch, C.E., 1979a. Australia's oldest known ornaments. Antiquity 53, 39-43.

https://doi.org/10.1017/S0003598X00042009

Dortch, C.E., 1979b. Devil's Lair, an example of prolonged cave use in south-western Australia. World Archaeology 10(3), 258-279. https://doi.org/10.1080/00438243.1979.9979736

Dortch, C.E., 1980. A possible pendant of marl from Devil's Lair, Western Australia. Rec. West. Aust. Mus. 8(3), 401-403.

Dortch, C.E., 1986. Correct provenance and radiocarbon age of an 'early phase' grindstone, Miriwun Rockshelter, Kimberley, northwest Australia. Aust. Archaeol. 23, 85-86.

Dragovich, D., 1986. Minimum age of some desert varnish near Broken Hill, New South Wales. Search 17, 149-151.

Dubin, L.S., 1987. The History of Beads: From 30,000 B.C. to the Present. H.N. Abrams, New York.

Faris, J.C., 1972. Nuba Personal Art. Duckworth, London.

Feary, S., 1996. An Aboriginal burial with grave goods near Cooma, New South Wales. Aust. Archaeol. 43, 40-42. https://doi.org/10.1080/03122417.1996.11681576

Flood, J., 1995. Archaeology of the Dreamtime: The Story of Prehistoric Australia and its People. Rev. ed. Angus \& Robertson, Pymble.

Flood, J., 1997. Rock Art of the Dreamtime: Images of Ancient Australia. Angus and Robertson, Pymble.

Franklin, N., Habgood, P., 2007. Modern human behaviour and Pleistocene Sahul in review. Aust. Archaeol. 65, 1-16. https://doi.org/10.1080/03122417.2007.11681854

Freedman, L., Lofgren, M., 1983. Human skeletal remains from Lake Tandou, New South Wales. Archaeol. in Oceania 18(2), 98-105. https://doi.org/10.1002/arco.1983.18.2.98

Fullagar, R., 2006. Starch grains, stone tools and modern hominin behaviour, in: Ulm, S., Lilley, I., (Eds), An Archaeological Life: Papers in Honour of Jay Hall. Aboriginal and Torres Strait Islander Studies Unit, University of Queensland, Brisbane, pp. 191-202.

Fullagar, R., David, B., 1997. Investigating changing attitudes towards an Australian Aboriginal Dreaming mountain over $>37,000$ years of occupation via residue and use wear analyses of stone artefacts. Camb. Archaeol. J. 7(1), 139-144. https://doi.org/10.1017/S0959774300001517

Gao, X., 2013. Paleolithic cultures in China. uniqueness and divergence. Curr. Anthropol. 54(S8), S358S370. https://doi.org/10.1086/673502

Geneste, J.-M., David, B., Plisson, H., Clarkson, C., Delannoy, J.-J., Petchey, F., Whear, R., 2010. Earliest

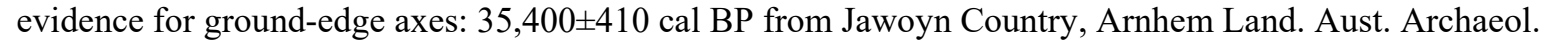
71, 66-69. https://doi.org/10.1080/03122417.2010.11689385

Gillespie, R., 1997. On human blood, rock art and calcium oxalate: further studies on organic carbon content and radiocarbon age of materials relating to Australian rock art. Antiquity 71, 430-437. https://doi.org/10.1017/S0003598X00085033

Gillespie, R., Roberts, R.G. 2000. On the reliability of age estimates for human remains at Lake Mungo. J. Hum. Evol. 38(5), 727-732. https://doi.org/10.1006/jhev.1999.0398 
Gilligan, I., 2007a. Clothing and modern human behaviour: prehistoric Tasmania as a case study. Archaeol. in Oceania 42(3), 102-111. https://doi.org/10.1002/j.1834-4453.2007.tb00023.x

Gilligan, I., 2007b. Resisting the cold in ice age Tasmania: thermal environment and settlement strategies. Antiquity 81, 555-568. https://doi.org/10.1017/S0003598X00095582

Gilligan, I., 2007c. Another Tasmanian Paradox: Clothing and Thermal Adaptations in Aboriginal Australia. BAR International Series S1710. Archaeopress, Oxford.

Glover, I.C., 1981. Leang Burung 2: an Upper Palaeolithic rock shelter in south Sulawesi, Indonesia. Mod. Quat. Res. Southeast Asia 6, 1-38.

Habgood, P.J., Franklin, N.R., 2008. The revolution that didn't arrive: a review of Pleistocene Sahul. J. Hum. Evol. 55(2), 187-222. https://doi.org/10.1016/j.jhevol.2007.11.006

Hamm, G., Mitchell, P., Arnold, L.J., Prideaux, G.J., Questiaux, D., Spooner, N.A., Levchenko, V.A., Foley, E.C., Worthy, T.H., Stephenson, B., Coulthard, V., Coulthard, C., Wilton, S., Johnston, D., 2016. Cultural innovation and megafauna interaction in the early settlement of arid Australia. Nature 539, 280-283. https://doi.org/10.1038/nature20125

Harris, S., Ranson, D., Brown, S., 1988. Maxwell River archaeological survey 1986. Aust. Archaeo. 27, 8997.

Harvati, K., Röding, C., Bosman, A.M., Karakostis, F.A., Grün, R., Stringer, C., Karkanas, P., Thompson, N.C., Koutoulidis, V., Moulopoulos, L.A. and Gorgoulis, V.G., 2019. Apidima Cave fossils provide earliest evidence of Homo sapiens in Eurasia. Nature, p.1.

Henshilwood, C.S., Marean, C.W., 2003. The origin of modern human behavior: critique of the models and their test implications. Curr. Anthropol. 44(5), 627-651. https://doi.org/10.1086/377665

Hershkovitz, I., Weber, G.W., Quam, R., Duval, M., Grün, R., Kinsley, L., Ayalon, A., Bar-Matthews, M., Valladas, H., Mercier, N. and Arsuaga, J.L., 2018. The earliest modern humans outside Africa. Science, 359(6374), pp.456-459.

Hiscock, P., 2017. Discovery curves, colonisation and Madjedbebe. Aust. Archaeol. 83(3), 168-171. https://doi.org/10.1080/03122417.2017.1408544

Hiscock, P., O'Connor, S., 2005. Arid paradises or dangerous landscapes: a review of explanations for Paleolithic assemblage change in arid Australia and Africa, in: Veth, P., Smith, M., Hiscock, P., (Eds), Desert Peoples: Archaeological Perspectives. Blackwell, Malden, pp. 58-77. https://doi.org/10.1002/9780470774632.ch4

Hiscock, P., O'Connor, S., 2006. An Australian perspective on modern behaviour and artefact assemblages. Before Farming 2006(2), 1-10. https://doi.org/10.3828/bfarm.2006.2.4

Hiscock, P., O’Connor, S., Balme, J., Maloney, T., 2016. World's earliest ground-edge axe production coincides with human colonisation of Australia. Aust. Archaeol. 82, 2-11.

https://doi.org/10.1080/03122417.2016.1164379

Hodder, I., 1979. Economic and social stress and material culture patterning. Am. Ant. 44(3), 446-454. https://doi.org/10.2307/279544

Hodder, I., 1991. The Meanings of Things. Material Culture and Symbolic Expressions. Harper Collins, London. 
Hogg, A.G., Hua, Q., Blackwell, P.G., Niu, M., Buck, C.E., Guilderson, T.P., Heaton, T.J., Palmer, J.G., Reimer, P.J., Reimer, R.W., Turney, C.S.M., Zimmerman, S.R.H., 2013. SHCal13 Southern Hemisphere calibration, 0-50,000 years cal BP. Radiocarbon 55(4), 1889-1903.

https://journals.uair.arizona.edu/index.php/radiocarbon/article/view/16783

Hovers, E., Ilani, S., Bar-Yosef, O., Vandermeersch, B., 2003. An early case of color symbolism: ochre use by modern humans in Qafzeh Cave. Curr. Anthropol. 44(4), 491-522. https://doi.org/10.1086/375869

Hua Q, Barbetti M, Levchenko V.A., D’Arrigo, R.D., Buckley, B.M., Smith, A.M., 2012. Monsoonal influence on Southern Hemisphere 14CO2. Geophysical Research Letters 39(19), L19806.

https://doi.org/10.1029/2012GL052971

Huang, W., Hou, Y., Gao, L., 2009. "Western elements" in the Chinese Paleolithic as viewed in a framework of early human cultural evolution. Acta Anthropol. Sinica 28(1), 16-25.

Hublin, J.-J., Ben-Ncer, A., Bailey, S.E., Freidline, S.E., Neubauer, S., Skinner, M.M., Bergmann, I., Le Cabec, A., Benazzi, S., Harvati, K., Gunz, P., 2017. New fossils from Jebel Irhoud, Morocco and the panAfrican origin of Homo sapiens. Nature 546, 289-292. https://doi.org/10.1038/nature22336

Hunt, C., Barker, G., 2014. Missing links, cultural modernity and the dead: anatomically Modern Humans in the Great Cave of Niah (Sarawak, Borneo), in: Dennell, R., Porr, M., (Eds.), Southern Asia, Australia, and the Search for Human Origins. Cambridge University Press, Cambridge, pp. 90-107.

https://doi.org/10.1017/CBO9781139084741.008

Ji, X., Kuman, K., Clarke, R.J., Forestier, H., Yinghua, L., Ma, J., Qui K., Li, H., Wu, Y., 2016. The oldest Hoabinhian technocomplex in Asia (43.5 ka) at Xiaodong rockshelter, Yunnan Province, southwest China. Quat. Int. 400, 166-174. https://doi.org/10.1016/j.quaint.2015.09.080

Jones, R., Cosgrove, R., Allen, J., Cane, S., Kiernan, K., Webb, S., Loy, T., West, D., Stadler, E., 1988. An archaeological reconnaissance of karst caves within the southern forests region of Tasmania, September 1987. Aust. Archaeol. 26, 1-23.

Jones, R., Johnson, I., 1985. Deaf Adder Gorge: Lindner Site, Nauwalabila I, in: Jones, R. (Ed.), Archaeological Research in Kakadu National Park. Special Publication 13, Australian National Parks and Wildlife Service, Canberra, pp. 165-227.

Joordens, J.C.A., d'Errico, F., Wesselingh, F.P., Munro, S., de Vos, J., Wallinga, J., Ankjærgaard, C., Reimann, T., Wijbrans, J.R., Kuiper, K.F., Mücher, H.J., Coqueugniot, H., Prié, V., Joosten, I., van Os, B., Schulp, A.S., Panuel, M., van der Haas, V., Lustenhouwer, W., Reijmer, J.J.G., Roebroeks, W. 2015. Homo erectus at Trinil on Java used shells for tool production and engraving. Nature 518, 228-231.

https://doi.org/10.1038/nature13962

Klein, R.G., Edgar, B., 2002. The Dawn of Human Culture. Wiley, New York.

Kuhn, S.L., Stiner, M.C., 2007a. Body ornamentation as information technology: towards an understanding of the significance of early beads, in: Mellars, P., Boyle, K., Bar-Yosef, O., Stringer, C., (Eds.), Rethinking the Human Revolution: New Behavioural and Biological Perspectives on the Origin and Dispersal of Modern Humans. McDonald Institute for Archaeological Research, University of Cambridge, Cambridge, pp. $45-54$.

Kuhn, S.L., Stiner, M.C., 2007b. Paleolithic ornaments: implications for cognition, demography and identity. Diogenes 54(2), 40-48. https://doi.org/10.1177\%2F0392192107076870 
Kuper, H., 1973. Costume and identity. Comp. Stud. Soc. Hist. 15(3), 348-367.

https://doi.org/10.1017/S0010417500007143

Langley, M.C., 2013. Storied landscapes make us (modern) human: landscape socialisation in the Palaeolithic and consequences for the archaeological record. J. Anthropol. Archaeol. 32(4), 614-629. https://doi.org/10.1016/j.jaa.2013.10.001

Langley, M.C., Clarkson, C., Ulm, S., 2011. From small holes to grand narratives: the impact of taphonomy and sample size on the modernity debate in Australia and New Guinea. J. Hum. Evol. 61(2), 197-208. https://doi.org/10.1016/j.jhevol.2011.03.002

Langley, M.C., O'Connor, S., 2015. 6,500-year-old Nassarius shell appliqués in Timor-Leste: technological and use wear analyses. J. Archaeol. Sci. 62, 175-192. https://doi.org/10.1016/j.jas.2015.06.012

Langley, M.C., O’Connor, S., 2016. An enduring shell artefact tradition from Timor-Leste: Oliva bead production from the Pleistocene to late Holocene at Jerimalai, Lene Hara, and Matja Kuru 1 and 2. PLOS ONE 11(8), e0161071. https://doi.org/10.1371/journal.pone.0161071

Langley, M.C., O’Connor, S., 2019. 40,000 years of ochre utilization in Timor-Leste: powders, prehensile traces, and body painting. Palaeoanthropol. 2019, 82-104. https://doi.org/10.4207/PA.2019.ART125

Langley, M.C., O’Connor, S., Aplin, K., 2016a. A >46,000-year-old kangaroo bone implement from Carpenter's Gap 1 (Kimberley, northwest Australia). Quat. Sci. Rev. 154, 199-213.

https://doi.org/10.1016/j.quascirev.2016.11.006

Langley, M.C., O’Connor, S., Piotto, E., 2016b. 42,000-year-old worked and pigment-stained Nautilus shell from Jerimalai (Timor-Leste): evidence for an early coastal adaptation in ISEA. J. Hum. Evol. 97, 1-16. https://doi.org/10.1016/j.jhevol.2016.04.005

Langley, M.C., Litster, M., Wright, D., May, S.K., (Eds), 2018. The Archaeology of Portable Art: Southeast Asia, Pacific, and Australian Perspectives. Routledge, Oxon.

Langley, M.C., Rybachuk, M. (in prep). Re-analysis of the 'engraved' Diprotodon tooth from Spring Creek (Victoria).

Leavesley, M.G., 2007. A shark-tooth ornament from Pleistocene Sahul. Antiquity 81, 308-315. https://doi.org/10.1017/S0003598X00095193

Leavesley, M., Allen, J., 1998. Dates, disturbance and artefact distributions: another analysis of Buang Merabak, a Pleistocene site on New Ireland, Papua New Guinea. Archaeol. in Oceania 33(2), 63-82. https://doi.org/10.1002/j.1834-4453.1998.tb00405.x

Leavesley, M.G., Bird, M.I., Fifield, L.K., Hausladen, P.A., Santos, G.M., di Tada, M.L., 2002. Buang Merabak: early evidence for human occupation in the Bismarck Archipelago, Papua New Guinea. Aust. Archaeol. 54, 55-57. https://doi.org/10.1080/03122417.2002.11682070

Lemonnier, P., 1992. Elements for an Anthropology of Technology. Anthropological Papers 88. Museum for Anthropology, University of Michigan, Ann Arbor.

Lewis, H., Paz, V., Lara, M., Barton, H., Piper, P., Ochoa, J., Vitales, T., Carlos, A.J., Higham T., Neri, L., Hernandez, V., Stevenson, J., Robles, E.C., Ragragio, A., Padilla, R., Solheim, W., Ronquillo, W., 2008. Terminal Pleistocene to mid-Holocene occupation and an early cremation burial at Ille Cave, Palawan, Philippines. Antiquity 82, 318-335. https://doi.org/10.1017/S0003598X00096836 
Lorblanchet, M., 1992. The rock engravings of Gum Tree Valley and Skew Valley, Dampier, Western Australia: Chronology and functions of the sites, in: McDonald, J., Hascovec, L.P., (Eds), State of the Art: Regional Rock Art Studies in Australia and Melanesia. Occasional AURA Publication 6. Australian Rock Art Research Association, Melbourne, pp. 39-59.

Loy, T.H., Jones, R., Nelson, D.E., Meehan, B., Vogel, J., Southon, J., Cosgrove, R., 1990. Accelerator radiocarbon dating of human blood proteins in pigments from late Pleistocene art sites in Australia. Antiquity 64, 110-116. https://doi.org/10.1017/S0003598X00077346

Macintosh, N.W.G., Smith, K.N., Bailey, A.B., 1970. Lake Nitchie skeleton-unique Aboriginal burial. Archaeol. Phys. Anthropol. in Oceania 5(2), 85-101.

Marean, C.W., Bar-Matthews, M., Bernatchez, J., Fisher, E., Goldberg, P., Herries, A.I.R., Jacobs, Z., Jerardino, A., Karkanas, P., Minichillo, T., Nilssen, P.J., Thompson, E., Watts, I., Williams, H.M., 2007. Early human use of marine resources and pigment in South Africa during the Middle Pleistocene. Nature 449, 905-908. https://doi.org/10.1038/nature06204

Marwick, B., 2018. The Hoabinhian of Southeast Asia and its relationship to regional Pleistocene lithic technologies, in: Robinson, E., Sellet, F., (Eds), Lithic Technological Organization and Paleoenvironmental Change: Global and Diachronic Perspectives. Studies in Human Ecology and Adaptation 9. Springer, Cham, pp. 63-78. https://doi.org/10.1007/978-3-319-64407-3_4

McBrearty, S., Brooks, A.S., 2000. The revolution that wasn't: a new interpretation of the origin of modern human behavior. J. Hum. Evol. 39(5), 453-563. https://doi.org/10.1006/jhev.2000.0435

McDonald, J., Officer, K., Jull, T., Donahue, D., Head, J., Ford, B., 1990. Investigating 14C AMS: dating prehistoric rock art in the Sydney Sandstone Basin, Australia. Rock Art Research 7(2), 83-92.

McDonald, J., Reynen, W., Petchey, F., Ditchfield, K., Byrne, C., Vannieuwenhuyse, D., Leopold, M., Veth, P., 2018. Karnatukul (Serpent's Glen): a new chronology for the oldest site in Australia's Western Desert. PLOS ONE 13(10), e0202511. https://doi.org/10.1371/journal.pone.0202511

McGowan, A., Shreeve, B., Brolsma, H., Hughes, C., 1993. Photogrammetric recording of Pleistocene cave paintings in southwest Tasmania, in: Smith, M.A., Spriggs, M., Fankhauser, B., (Eds), Sahul in Review: Pleistocene Archaeology in Australia, New Guinea and Island Melanesia. Occasional Papers in Prehistory 24. Department of Prehistory, Research School of Pacific Studies, Australian National University, Canberra, pp. 225-232.

Meehan, B., 1984. Aboriginal skeletal remains. Aust. Archaeol. 19, 122-147.

Mellars, P., 2006. Going east: new genetic and archaeological perspectives on the modern human colonization of Eurasia. Science 313, 796-800. https://doi.org/10.1126/science.1128402

Morse, K., 1993. Shell beads from Mandu Mandu Creek Rock-Shelter, Cape Range Peninsula, Western Australia, dated before 30,000 b.p. Antiquity 67, 877-883. https://doi.org/10.1017/S0003598X00063894

Morwood, M., 1978. Ken's Cave and the art of central-western Queensland. Aust. Archaeol. 8, 22-31.

Morwood, M.J., 1982. Early man in north Queensland. Aust. Archaeol. 15, 92-96.

Morwood, M., 1989. The archaeology of Aboriginal art in S.E. Cape York: a research proposal. Rock Art Res. 6(1), 71-72. 
Morwood, M.J., 2002. Visions from the Past: The Archaeology of Australian Aboriginal Art. Allen and Unwin, St Leonards.

Morwood, M.J., Hobbs, D.R., Price, D.M., 1995. Excavations at Sandy Creek 1 and 2, in: Morwood, M.J., Hobbs, D.R., (Eds), Quinkan Prehistory: The Archaeology of Aboriginal Art in S.E. Cape York Peninsula, Australia. Tempus 3. Anthropology Museum, University of Queensland, St Lucia, pp. 71-92.

Morwood, M.J., Jung, S., 1995. Excavations at Magnificent Gallery, in: Morwood, M.J., Hobbs, D.R., (Eds), Quinkan Prehistory: The Archaeology of Aboriginal Art in S.E. Cape York Peninsula, Australia. Tempus 3. Anthropology Museum, University of Queensland, St Lucia, pp. 93-100.

Morwood, M.J. and P.J. Trezise 1989 Edge-ground axes in Pleistocene Greater Australia: new evidence from S.E. Cape York Peninsula. Queensland Archaeological Research 6, 77-90.

https://doi.org/10.25120/qar.6.1989.138

Movius, H.L. Jr., 1944. Early Man and Pleistocene Stratigraphy in Southern and Eastern Asia. Papers of the Peabody Museum of American Archaeology and Ethnology 19(3). Peabody Museum, Cambridge.

Movius, H.L. Jr., 1948. The Lower Palaeolithic cultures of southern and eastern Asia. Trans. Am. Phil. Soc. 38(4), 329-420.

Mulvaney, K., 2013. Iconic imagery: Pleistocene rock art development across northern Australia. Quat. Int. 285, 99-110. https://doi.org/10.1016/j.quaint.2011.07.020

Nanson, G.C., Young, R.W., Stockon, E.D., 1987. Chronology and palaeoenvironments of the Cranebrook Terrace (near Sydney) containing artefacts more than 40,000 years. Archaeol. in Oceania 22(2), 72-78. https://doi.org/10.1002/j.1834-4453.1987.tb00168.x

Nelson, D.E., 1993. Second thoughts on a rock-art date. Antiquity 67, 893-895.

https://doi.org/10.1017/S0003598X00063924

Nobbs, M., Dorn, R., 1993. New surface exposure ages for petroglyphs from the Olary Province, South Australia. Archaeol. in Oceania 28(1), 18-39. https://doi.org/10.1002/j.1834-4453.1993.tb00311.x

Norton, C.J., Jin, J.J.H., 2009. The evolution of modern human behavior in East Asia: current perspectives. Evol. Anthropol. 18(6), 247-260. https://doi.org/10.1002/evan.20235

O'Connell, J.F., Allen, J., 2007. Pre-LGM Sahul (Pleistocene Australia-New Guinea) and the archaeology of early modern humans, in: Mellars, P., Boyle, K., Bar-Yosef, O., Stringer, C., (Eds.), Rethinking the Human Revolution: New Behavioural and Biological Perspectives on the Origin and Dispersal of Modern Humans. McDonald Institute for Archaeological Research, University of Cambridge, Cambridge, pp. 395-410.

O’Connell, J.F., Allen, J., Williams, M.A.J., Williams, A.N., Turney, C.S.M., Spooner, N.A., Kamminga, J., Brown, G., Cooper, A., 2018. When did Homo sapiens first reach Southeast Asia and Sahul? Proc. Nat. Acad. Sci. USA 115, 8482-8490. https://doi.org/10.1073/pnas.1808385115

O’Connor, S., 1999. 30,000 Years of Aboriginal Occupation: Kimberley, North West Australia. Terra Australis 14. Archaeology and Natural History Publications and Centre for Archaeological Research, Australian National University, Canberra.

O'Connor, S., 2007. New evidence from East Timor contributes to our understanding of earliest modern human colonisation east of the Sunda shelf. Antiquity 81, 523-535.

https://doi.org/10.1017/S0003598X00095569 
O'Connor, S., 2010. Continuity in shell artefact production in Holocene East Timor, in: Bellina, B., Bacus, E.A., Pryce, T.O., Christie, J.W., (Eds), 50 Years of Archaeology in Southeast Asia: Essays in Honour of Ian Glover. River Books, Bangkok, pp. 219-233.

O'Connor, S., Aplin, K., Pasveer, J., Hope, G., 2005a. Liang Nabulei Lisa: A late Pleistocene and Holocene sequence from the Aru Islands, in: O'Connor, S., Spriggs, M., Veth, P., (Eds), The Archaeology of the Aru Islands, Eastern Indonesia. Terra Australis 22. Canberra, ANU E Press, pp. 125-161.

O’Connor, S., Aplin, K., Szabó, K., Pasveer, J., Veth, P., Spriggs, M., 2005b. Liang Lemdubu: A Pleistocene cave site in the Aru Islands, in: O'Connor, S., Spriggs, M., Veth, P., (Eds), The Archaeology of the Aru Islands, Eastern Indonesia. Terra Australis 22. Canberra, ANU E Press, pp. 171-204.

O’Connor, S., Barham, A., Spriggs, M., Veth, P., Aplin, K., St Pierre, E., 2010. Cave archaeology and sampling issues in the tropics: a case study from Lene Hara Cave, a 42,000 year old occupation site in East Timor, Island Southeast Asia. Aust. Archaeol. 71, 29-40. https://doi.org/10.1080/03122417.2010.11689382

O'Connor, S., Ono, R., Clarkson, C., 2011. Pelagic fishing at 42,000 years before the present and the maritime skills of modern humans. Science 334, 1117-1121.

O'Connor, S., Fankhauser, B., 2001. Art at 40,000 BP? One step closer: an ochre covered rock from Carpenter's Gap Shelter 1, Kimberley region, Western Australia, in: Anderson, A., Lilley, I., O'Connor, S., (Eds), Histories of Old Ages: Essays in Honour of Rhys Jones. Pandanus Books, Canberra, pp.287-300.

O'Connor, S., Langley, M.C., 2018. The contribution of early Southeast Asian material to a global understanding of portable art, in: Langley, M.C., Litster, M., Wright, D., May, S.K., (Eds), The Archaeology of Portable Art: Southeast Asian, Pacific, and Australian Perspectives. Routledge, Oxon, pp. 13-24.

O'Hanlon, M., 1989. Reading the Skin: Adornment, Display, and Society among the Wahgi. Trustees of the British Museum, London.

Ossa, P., Marshall, B., Webb, C., 1995. New Guinea II Cave: A Pleistocene site on the Snowy River, Victoria. Archaeol. in Oceania 30(1), 22-35. https://doi.org/10.1002/j.1834-4453.1995.tb00325.x

Oyston, B. 1996. Thermoluminescence age determinations for the Mungo III human burial, Lake Mungo, southeastern Australia. Quat. Sci. Rev. 15(7), 739-749. https://doi.org/10.1016/0277-3791(96)00025-X

Page, K., Dare-Edwards, T., Thorne, A., Webb, S., Price, D., 1994. Pleistocene human occupation site at Lake Urana, New South Wales. Aust. Archaeol. 38, 38-44.

https://doi.org/10.1080/03122417.1994.11681518

Pardoe, C. 1993a. The Pleistocene is still with us: analytical constraints and possibilities for the study of ancient human remains in archaeology, in: Smith, M.A., Spriggs, M., Fankhauser, B., (Eds), Sahul in Review: Pleistocene Archaeology in Australia, New Guinea and Island Melanesia. Occasional Papers in Prehistory 24. Department of Prehistory, Research School of Pacific Studies, Australian National University, Canberra, pp. 81-94.

Pardoe, C., 1993b. Wamba yadu, a later Holocene cemetery of the central River Murray. Archaeol. in Oceania 28(2), 77-84. https://doi.org/10.1002/j.1834-4453.1993.tb00318.x

Pawlik, A., 2005. Detecting traits of modern behavior through microwear analysis: A case study from the Philippine Terminal Pleistocene, in: Kaifu, Y., Izuho, M., Goebel, T., Sato, H., Ono, A., (Eds), Emergence and Diversity of Modern Human Behavior in Paleolithic Asia. Texas A\&M University Press, College Station, pp. 182-198. 
Petchey, F., Ulm, S. 2012. Marine reservoir variation in the Bismarck region: an evaluation of spatial and temporal change in $\square \mathrm{R}$ and $\mathrm{R}$ over the last 3000 years. Radiocarbon 54(1), 45-58.

https://doi.org/10.2458/azu_js_rc.v54i1.13050

Pookajorn, S., 1996. Human activities and environmental changes during the late Pleistocene to middle Holocene in southern Thailand and Southeast Asia, in: Straus, L.G., Eriksen, B.V., Erlandson, J.M., Yesner, D.R., (Eds), Humans at the End of the Ice Age: The Archaeology of the Pleistocene-Holocene Transition. Plenum Press, London, pp. 201-213.

Pretty G.L., 1977. The cultural chronology of the Roonka Flat, in: Wright, R.V.S., (Ed.), Stone Tools as Cultural Markers: Change, Evolution and Complexity. Australian Institute of Aboriginal Studies, Canberra, pp. 288-331.

Pyatt, F.B., Barker, G.W., Rabett, R.J., Szabó, K., Wilson, B., 2010. Analytical examination of animal remains from Borneo: The painting of bone and shell. J. Archaeol. Sci. 37, 2102-2105. https://doi.org/ doi:10.1016/j.jas.2010.02.006

Rabett, R.J., Piper, P.J., 2012. The emergence of bone technologies at the end of the Pleistocene in southeast Asia: Regional and evolutionary implications. Camb. Archaeol. J. 22(1), 37-56. https://doi.org/ $10.1017 /$ S0959774312000030

Reimer, P.J., Bard, E., Bayliss, A., Beck, J.W., Blackwell, P.G., Bronk Ramsey, C., Buck, C.E., Cheng, H., Edwards, R.L., Friedrich, M., Grootes, P.M., Guilderson, T.P., Haflidason, H., Hajdas, I., Hatté, C., Heaton, T.J., Hoffmann, D.L., Hogg, A.G., Hughen, K.A., Kaiser, K.F., Kromer, B., Manning, S.W., Niu, M., Reimer, R.W., Richards, D.A., Scott, E.M., Southon, J.R., Staff, R.A., Turney, C.S.M., van der Plicht, J., 2013. INTCAL13 and MARINE13 radiocarbon age calibration curves 0-50,000 years cal BP. Radiocarbon 55(4), 1869-1887. https://doi.org/10.2458/azu_js_rc.55.16947

Reynen, W., 2018. Rockshelters and Human Mobility during the Last Glacial Maximum in the Pilbara Uplands, North-Western Australia. Unpublished PhD thesis, School of Social Sciences, University of Western Australia, Perth.

Reynolds, T., Barker, G., Barton, H., Cranbrook, G., Farr, L., Hunt, C., Kealhofer, L., Paz, V., Pike, A., Piper, P.J., Rabett, R.J., Rushworth, G., Stimpson, C., Szabó, K., 2013. The first modern humans at Niah, c.50,000-35,000 years ago, in: Barker, G. (Ed.), Rainforest Foraging and Farming in Island Southeast Asia. The Archaeology of the Niah Caves, Sarawak, Volume 1. McDonald Institute for Archaeological Research, Cambridge, pp. 135-172.

Roberts, R.G., Jones, R., Smith, M.A., 1993. Optical dating at Deaf Adder Gorge, Northern Territory, indicates human occupation between 53,000 and 60,000 years ago. Aust. Archaeol. 37, 58-59. https://doiorg.elibrary.jcu.edu.au/10.1080/03122417.1993.11681497

Roberts, R.G., Jones, R., Spooner, N.A., Head, M.J., Murray, A.S., Smith, M.A., 1994 The human colonisation of Australia: optical dates of 53,000 and 60,000 years bracket human arrival at Deaf Adder Gorge, Northern Territory. Quat. Sci. Rev. 13(5-7), 575-583. https://doi.org/10.1016/0277-3791(94)90080-9

Rosenfeld, A., 1991. Panaramittee: Dead or alive?, in: Bahn, P.G., Rosenfeld, A., (Eds), Rock Art and Prehistory. Oxbow, Oxford, pp. 136-144.

Rosenfeld, A., 1993. A review of the evidence for the emergence of rock art in Australia, in: Smith, M.A., Spriggs, M., Fankhauser, B., (Eds), Sahul in Review: Pleistocene Archaeology in Australia, New Guinea and Island Melanesia. Occasional Papers in Prehistory 24. Australian National University, Canberra, pp. 71-80. 
Rosenfeld, A., 1999. Rock art and rock markings. Aust. Archaeol. 49, 28-33.

https://doi.org/10.1080/03122417.1999.11681653

Rosenfeld, A., Horton, D., Winter, J., 1981. Early Man in North Queensland: Art and Archaeology in the Laura Area. Terra Australis 6. Department of Prehistory, Research School of Pacific Studies, Australian National University, Canberra.

Rosenfeld, A., Smith, M.A., 2002. Rock-art and the history of Puritjarra Rock Shelter, Cleland Hills, Central Australia. Proceedings of the Prehistoric Society 68, 103-124. https://doi.org/10.1017/S0079497X00001468

Schrire, C., 1982. The Alligator Rivers: Prehistory and Ecology in Western Arnhem Land. Terra Australis 7. Department of Prehistory, Research School of Pacific Studies, Australian National University, Canberra.

Sciama, L.D., Eicher, J.B. (Eds), 1998. Beads and Beadmakers: Gender, Material Culture and Meaning. Berg, Oxford.

Sim, R., 1998. The Archaeology of Isolation? Prehistoric Occupation in the Furneaux Group of Islands, Bass Strait, Tasmania. Unpublished PhD thesis, Department of Archaeology and Natural History, Australian National University, Canberra.

Sim, R., Thorne, A., 1990. Pleistocene human remains from King Island, southeastern Australia. Aust. Archaeol. 31, 44-51. https://doi.org/10.1080/03122417.1990.11681387

Simanjuntak, T., Sémah, F., Sémah, A.-M., 2015. Tracking evidence for modern human behavior in Paleolithic Indonesia, in: Kaifu, Y., Izuho, M., Goebel, T., Sato, H., Ono, A., (Eds), Emergence and Diversity of Modern Human Behavior in Paleolithic Asia. Texas A\&M University Press, College Station, pp. $158-170$.

Slack, M.J., Fullagar, R.L.K., Field, J.H., Border, A., 2004. New Pleistocene ages for backed artefact technology in Australia. Archaeol. in Oceania 39(3), 131-137. https://doi.org/10.1002/j.18344453.2004.tb00569.x

Smith, M.A., 2006. Characterizing late Pleistocene and Holocene stone artefact assemblages from Puritjarra Rock Shelter: a long sequence from the Australian desert. Rec. Aust. Mus. 58, 371-410.

https://doi.org/10.3853/j.0067-1975.58.2006.1470

Smith, M., 2013. The Archaeology of Australia’s Deserts. Cambridge University Press, Cambridge.

Smith, M.A., Fankhauser, B., Jercher, M., 1998. The changing provenance of red ochre at Puritjarra Rock Shelter, Central Australia: Late Pleistocene to present. Proceedings of the Prehistoric Society 64, 275-292. https://doi.org/10.1017/S0079497X00002243

Smith, M.A., Taçon, P.S.C., Curnoe. D., Thorne, A., 2007. Human dispersal into Australasia. Science 315, 597-598. https://doi.org/10.1126/science.315.5812.597b

Smith, M.A., Veth, P.M., 2004. Radiocarbon dates for baler shell in the Great Sandy Desert. Aust. Archaeol. 58, 37-38. https://doi.org/10.1080/03122417.2004.11681779

Smith, M.A., Watchman, A., Ross, J. 2009. Direct dating indicates a mid-Holocene age for archaic rock engravings in arid Central Australia. Geoarchaeology 24, 2, 191-203. https://doi.org/10.1002/gea.20262.

Stevenson, M.G., 1989. Sourdoughs and cheeckakos: the formation of identity-signaling social groups. J. Anthropol. Archaeol. 8(3), 270-312. https://doi.org/10.1016/0278-4165(89)90016-0 
Stone, T., Cupper, M.L., 2003. Last Glacial Maximum ages for robust humans at Kow Swamp, southern Australia. J. Hum. Evol. 45(2), 99-111. https://doi.org/10.1016/S0047-2484(03)00087-3

Strathern, A., Strathern, M., 1971. Self-decoration in Mount Hagen. Duckworth, London.

Szabó, K., Brumm, A., Bellwood, P., 2007. Shell artefact production at 32,000-28,000 BP in Island Southeast Asia. Thinking across media? Curr. Anthropol. 48(5), 701-723. https://doi.org/10.1086/520131

Taçon, P.S.C., 2008. Marks of possession: the archaeology of territory and cross- cultural encounter in Australia and South Africa, in: David, B., Thomas, J. (Eds.), Handbook of Landscape Archaeology. Left Coast Press, Walnut Creek, pp. 218-227. https://doi.org/10.4324/9781315427737.ch20

Taçon, P., Chippindale, C., 1994. Australia's ancient warriors: Changing depictions of fighting in the rock art of Arnhem Land, N.T. Camb. Archaeol. J. 4(2), 211-248. https://doi.org/10.1017/S0959774300001086

Thorne, A.G., Macumber, P.G., 1972. Discoveries of Late Pleistocene man at Kow Swamp, Australia. Nature 238, 316-319. https://doi.org/10.1038/238316a0

Thorne, A., Grün, R., Mortimer, G., Spooner, N.A., Simpson, J.J., McCulloch, M., Taylor, L., Curnoe, D., 1999. Australia's oldest human remains: age of the Lake Mungo 3 skeleton. J. Hum. Evol. 36(6), 591-612. https://doi-org.elibrary.jcu.edu.au/10.1006/jhev.1999.0305

Tostevin, G.B., 2007. Social intimacy, artefact visibility and acculturation models of Neanderthal-modern human interaction, in: Mellars, P., Boyle, K., Bar-Yosef, O., Stringer, C. (Eds.), Rethinking the Human Revolution: New Behavioural and Biological Perspectives on the Origin and Dispersal of Modern Humans. McDonald Institute for Archaeological Research, University of Cambridge, Cambridge, pp. 341-357.

Ulm, S., 2006. Australian marine reservoir effects: A guide to $\square$ R values. Aust. Archaeol. 63, 57-60. https://doi.org/10.1080/03122417.2006.11681838

van den Bergh, G.D., Li, B., Brumm, A., Grün, R., Yurnaldi, D., Moore, M.W., Kurniawan, I., Setiawan, R., Aziz, F., Roberts, R.G., Suyono, Storey, M., Setiabudi, E., Morwood, M.J., 2016. Earliest hominin occupation of Sulawesi, Indonesia. Nature 529, 208-211. https://doi.org/10.1038/nature16448

Vanderwal, R., Fullagar, R., 1989. Engraved Diprotodon tooth from the Spring Creek locality, Victoria. Archaeol. in Oceania 24(1), 13-16. https://doi.org/10.1002/j.1834-4453.1989.tb00201.x

van Heekeren, H.R., 1972. The Stone Age of Indonesia. 2nd ed. The Hague: Martinus Ninhoff.

Veth, P., Ward, I., Manne, T., Ulm, S., Ditchfield, K., Dortch, J., Hook, F., Petchey, F., Hogg, A., Questiaux, D., Demuro, M., Arnold, L., Spooner, N., Levchenko, V., Skippington, J., Byrne, C., Basgall, M., Zeanah, D., Belton, D., Helmholz, P., Bajkan, S., Bailey, R., Placzek, C., Kendrick, P., 2017. Early human occupation of a maritime desert, Barrow Island, north-west Australia. Quat. Sci. Rev. 168, 19-29. http://doi.org/10.1016/j.quascirev.2017.05.002

Viet, N., 2015. First archaeological evidence of symbolic activities from the Pleistocene of Vietnam, in: Kaifu, Y., Izuho, M., Goebel, T., Sato, H., Ono, A., (Eds), Emergence and Diversity of Modern Human Behavior in Paleolithic Asia. Texas A\&M University Press, College Station, pp. 133-139.

Wadley, L., 2001. What is cultural modernity? A general view and a South African perspective from Rose Cottage Cave. Camb. Archaeol. J. 11(2), 201-221. https://doi.org/10.1017/S0959774301000117

Walshe, K.A., 1994. A Taphonomic Analysis of the Vertebrate Material from Allen's Cave: Implications for Australian Arid Zone Archaeology. Unpublished PhD thesis, Australian National University, Canberra. 
Walshe, K., 2017. Koonalda Cave, Nullarbor Plain, South Australia - Issues in optical and radiometric dating of deep karst caves. Geochronometria 44(1), 366-373. https://doi.org/10.1515/geochr-2015-0081.

Watchman. A., 1992. Doubtful dates for Karolta engravings. Aust. Aboriginal Stud. 1, 51-55.

Watts, I., 2010. The pigments from Pinnacle Point Cave 13B, Western Cape, South Africa. J. Hum. Evol. 59(3-4), 392-411. https://doi.org/10.1016/j.jhevol.2010.07.006

Westaway, K.E., Louys, J., Due Awe, R., Morwood, M.J., Price, G.J., Zhao, J.-x., Aubert, M., JoannesBoyau, R., Smith, T.M., Skinner, M.M., Compton, T., Bailey, R.M., van den Bergh, G.D., de Vos, J., Pike, A.W.G., Stringer, C., Saptomo, E.W., Rizal, Y., Zaim, J., Santoso, W.D., Trihascaryo, A., Kinsley, L., Sulistyanto, B. 2018. An early modern human presence in Sumatra 73,000-63,000 years ago. Nature 548, 322-325. https://doi.org/10.1038/nature23452

White, J.P., 1972. Ol Tumbuna: Archaeological Excavations in the Eastern Central Highlands, Papua New Guinea. Terra Australis 2. Department of Prehistory, Research School of Pacific Studies, Australian National University, Canberra.

White, R., 1992. Beyond art: Toward an understanding of the origins of material representation in Europe. Ann. Rev. Anthropol. 21, 537-564. https://doi.org/10.1146/annurev.an.21.100192.002541

Wiessner, P., 1983. Style and social information in Kalahari San projectile points. American Antiquity 48(2), 253-276. https://doi.org/10.2307/280450

Wiessner, P., 1984. Reconsidering the behavioral basis for style: A case study among the Kalahari San. J. Anthropol. Archaeol. 3(3), 190-234. https://doi.org/10.1016/0278-4165(84)90002-3

Williams, A.N., 2013. A new population curve for prehistoric Australia. Proc. R. Soc. B 280, 20130486. http://doi.org/10.1098/rspb.2013.0486

Williams, A.N., Ulm, S., Cook, A.R., Langley, M.C., Collard, M., 2013. Human refugia in Australia during the Late Glacial Maximum and terminal Pleistocene geospatial analysis of the 25-12 ka Australian archaeological record. J. Archaeol. Sci. 40, 4612-4625. http://dx.doi.org/10.1016/j.jas.2013.06.015

Williams, A.N., Ulm, S., Sapienza, T., Lewis, S., Turney, C.S.M., 2018. Sea-level change and demography during the last glacial termination and early Holocene across the Australian continent. Quat. Sci. Rev. 182, 144-154. https://doi.org/10.1016/j.quascirev.2017.11.030.

Wobst, M.H., 1977. Stylistic behavior and information exchange, in: Cleland, C.E. (Ed.), For the Director: Research Essays in Honor of James B. Griffin. Museum of Anthropology, University of Michigan, Ann Arbor, pp. 317-342.

Wright, R.V.S., 1971. Archaeology of the Gallus site, Koonalda Cave. Australian Institute of Aboriginal Studies, Canberra. 